JOURNAL OF THE

AMERICAN MATHEMATICAL SOCIETY

Volume 12, Number 2, April 1999, Pages 381-444

S 0894-0347(99)00294-5

\title{
A NEW PROOF OF D. POPESCU'S THEOREM ON SMOOTHING OF RING HOMOMORPHISMS
}

\author{
MARK SPIVAKOVSKY
}

Dedicated to Professor H. Hironaka on the occasion of his sixtieth birthday

\section{Contents}

$\S 1$. Introduction

$\S 2$. Formal smoothness, smoothness, Jacobian criterion and André homology

$\S 3$. Smoothing of algebras defined by linear homogeneous equations

$\S 4$. Smoothing of an isolated, almost complete intersection singularity with no residue field extension

$\S 5$. Smoothing of an isolated singularity over a local ring

with no residue field extension

$\S 6$. Separability in field extensions

$\S 7$. Residue field extensions

induced by formally smooth homomorphisms 414

$\S 8$. Smoothing of an isolated singularity over a local ring 420

$\S 9$. Smoothing of ring homomorphisms 428

$\S 10$. Smoothing in the category of subalgebras 430

§11. Approximation theorems 437

Appendix. Regular homomorphisms which are not injective 442

References

444

\section{$\S 1$. INTRODUCTION}

In this paper we give a new proof, and some strengthenings of the following theorem of D. Popescu:

Theorem 1.1 ([1], [13]-[16] and [18]). Let $A \stackrel{\sigma}{\rightarrow} B$ be a homomorphism of noetherian rings. The homomorphism $\sigma$ is regular if and only if $B$ is a filtered inductive limit of smooth A-algebras of finite type.

Received by the editors May 8, 1992 and, in revised form, July 24, 1998

1991 Mathematics Subject Classification. Primary 13B40, 13C10, 14B05, 14B12, $14 \mathrm{E} 40$.

Key words and phrases. Smooth homomorphism, Néron desingularization, Artin approximation.

Research supported by the Harvard Society of Fellows, NSF, NSERC and the Connaught Fund.

(C)1999 American Mathematical Society 
(See [10, Chapter 11 and Chapter 13, (33.A), p. 249] for the definition of regular and smooth homomorphisms and $\S 2$ for a discussion of some of their main properties.)

"If" is well known: see Popescu's argument in [18, Lemma 1.4], or apply André's theorem (Property 2.8 and Corollary 2.9 below) and the fact that André homology commutes with direct limits ([18, Lemma 3.2] and [2, Chapter III, Proposition 35]). Our main interest is in proving "only if".

Theorem 1.1 can be restated as follows. To say that $B$ is a filtered inductive limit of smooth finite type $A$-algebras is equivalent to saying that any commutative diagram

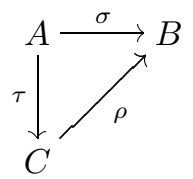

where $C$ is a finitely generated $A$-algebra, can be extended to a commutative diagram

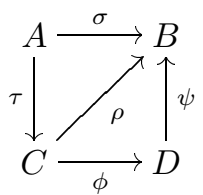

where $D$ is a smooth finitely generated $A$-algebra. Theorem 1.1 asserts that such an extension exists whenever $\sigma$ is a regular homomorphism.

There are two refinements of Theorem 1.1, conjectured by M. Artin in [4] at the same time with Theorem 1.1. The first [4, p. 225, Conjecture 2] is to require that the map $\phi$ in the diagram (1.2) be smooth wherever possible (roughly speaking, away from the non-smooth locus of $C$ ). In our proof, this requirement is satisfied by construction. Thus, what we actually prove is the following stronger version of Theorem 1.1.

For an $A$-algebra $C$ of finite type, let $H_{C / A}$ denote the Jacobian ideal of $C$ over $A$ (see Definition 2.11 for the definition of $H_{C / A}$ ). The ideal $H_{C / A}$ defines the nonsmooth locus of $C$ over $A$; in other words, for a prime ideal $P$ of $C, C_{P}$ is smooth over $A$ if and only if $H_{C / A} \not \subset P$ - see $\S 2$ for details.

Theorem 1.2. Consider a commutative diagram (1.1), where $\sigma$ is a regular homomorphism of noetherian rings. Then there exists a commutative diagram (1.2) such that $D$ is smooth of finite type over $A$ and $H_{C / A} B \subset \sqrt{H_{D / C} B}$.

This gives an affirmative answer to Conjecture 2 of [4].

The second direction in which Theorem 1.1 can be strengthened is

Problem 1.3 ([4, p. 224]). Assume that the homomorphism $\rho$ in diagram (1.1) is injective. Does there exist a diagram (1.2) with D smooth of finite type over A, such that $\psi$ is also injective? In other words, is $B$ a filtered inductive limit of smooth finitely generated A-subalgebras?

Note that Problem 1.3 makes sense even if $\sigma$ itself is not injective. See the Appendix for a discussion of non-injective regular homomorphisms. 
Problem 1.3 is stated in [4] in the case when $A$ is a field and $B=A\left[\left[x_{1}, \ldots, x_{n}\right]\right]$ is a formal power series ring over $A$. In fact, it turns out to have an affirmative answer for a wide class of regular homomorphisms. Namely, in $\S 10$ we give an affirmative solution to Problem 1.3 assuming that $A$ is reduced and for any minimal prime $Q$ of $B, \frac{B}{Q}$ has infinite transcendence degree over $\kappa(Q \cap A)$ (in particular, whenever $A$ is a reduced noetherian ring and $B=A\left[\left[x_{1}, \ldots, x_{n}\right]\right]$, or when $A$ is reduced, essentially of finite type over a field or $\mathbb{Z}$ and $B$ is the completion of $A$ along a nonzero ideal - see $\S 10$ for details). We give an example to show that the assumption of infinite transcendence degree is necessary. We also give an example of a diagram (1.1) with $\rho$ injective and $A$ non-reduced, such that there does not exist a diagram (1.2) with $\psi$ injective and $H_{C / A} B \subset \sqrt{H_{D / C} B}$. This shows that the hypothesis that $A$ is reduced is necessary, at least for this method of proof.

Two special cases of Theorem 1.1 are of particular interest for applications. The first is the case when $(A, I)$ is a Henselian pair such that the $I$-adic completion homomorphism $\sigma: A \rightarrow \hat{A}$ is regular (this is the case whenever $A$ is excellent and, more generally, whenever $A$ is a G-ring [10, (33.A) and (34.A)]). Applying Theorem 1.1 to $\sigma$ yields the general form of Artin approximation theorem (which was already known to follow from D. Popescu's theorem and is included here mainly for completeness), as well as a generalized version of the nested approximation theorem and B. Teissier's nested smoothing theorem (see $\S 11$ ).

The second special case of interest is the case when $B$ is a regular local ring and $A$ is a field or a Dedekind domain, contained in $B$, such that the inclusion map $A \rightarrow B$ is a regular homomorphism (if $B$ is equicharacteristic, we may take $A$ to be the prime field of $B$ ). Theorem 1.1, applied in this case, yields a positive answer to the Bass-Quillen conjecture in the equicharacteristic case, as well as to several related conjectures on freeness of projective modules (see [18] for details).

Conventions. All the rings in this paper will be commutative with 1 . We will denote by $\mathbb{N}$ the set of positive integers, by $\mathbb{N}_{0}$ the set of non-negative integers.

For an ideal $I, \sqrt{I}$ will denote the radical of $I$. Let $\sigma: A \rightarrow B$ be a homomorphism of rings. If $I$ is an ideal of $A$, we write $I B$ for $\sigma(I) B$. If $P$ is a subset of $B$, we write $P \cap A$ for $\sigma^{-1}(P)$. The module of relative Kähler differentials of $B$ over $A$ will be denoted by $\Omega_{B / A}$. An $A$-algebra $B$ will be said to be of finite type if it is finitely presented over $A$, essentially of finite type over $A$ if $B$ is a localization of a finite type $A$-algebra. A free $A$-algebra is a polynomial ring over $A$, on an arbitrary (not necessarily finite) set of generators. For a prime ideal $P$ in a ring $A, \kappa(P)$ will denote the residue field of $A_{P}$. If $A$ is a ring and $M$ an $A$-module, $S_{A} M$ will stand for the symmetric algebra over $M$. If $S$ is a multiplicative subset of $A, M_{S}$ will denote the localization of $M$ with respect to $S$, that is, $M_{S}=M \otimes_{A} A_{S}$. Similarly, if $P$ is a prime ideal of $A$, we will write $M_{P}$ for $M \otimes_{A} A_{P}$. If $m$ is an ideal in a ring $A, A n n_{A} m^{\infty}$ will stand for $\bigcup_{i=1}^{\infty} A n n_{A} m^{i}$. Let $A$ be a ring and $u=\left(u_{1}, \ldots, u_{n}\right)$ independent variables. Given $r \in \mathbb{N}_{0}$ and a subset $g=\left\{g_{1}, \ldots, g_{r}\right\} \subset A[u], \Delta_{g}$ will denote the ideal of $A[u]$ generated by all the $r \times r$ minors of the matrix $\left(\frac{\partial g}{\partial u}\right) \equiv\left(\frac{\partial g_{i}}{\partial u_{j}}\right)_{\substack{1 \leq i \leq r \\ 1 \leq j \leq n}}$. If $v$ is a subset of the variables $u, \Delta_{g, v}$ will stand for the ideal generated by all the $r \times r$ minors of the matrix $\left(\frac{\partial g}{\partial v}\right)$. If $g_{1}, \ldots, g_{r}$ are linear homogeneous, we will denote by $\Delta_{g}^{0}$ the ideal of $A$ generated 
by the $r \times r$ minors of $\left(\frac{\partial g_{i}}{\partial u_{j}}\right)_{\substack{1 \leq i \leq r \\ 1 \leq j \leq n}}$. In the case $r=0$, we adopt the convention that the determinant of the empty matrix is 1 .

We now outline the proof of Theorem 1.2. Our proof (as well as Popescu's original proof of Theorem 1.1) starts out with the following two observations, due to D. Popescu. Consider a diagram (1.1).

Lemma 1.4. Suppose that $H_{C / A} B=B$. Then there exists a diagram (1.2) with $D$ smooth of finite type over $A$ and such that $H_{C / A} D=H_{D / C}=D$.

Proof. Let $a_{1}, \ldots, a_{n}$ be a set of generators of $H_{C / A}$. Then there exist $b_{1}, \ldots, b_{n} \in B$ with $\sum_{i=1}^{n} \rho\left(a_{i}\right) b_{i}=1$. Let $T_{1}, \ldots, T_{n}$ be new variables and let $D=\frac{C\left[T_{1}, \ldots, T_{n}\right]}{\left(\sum_{i=1}^{n} a_{i} T_{i}-1\right)}$. Define the map $\psi: D \rightarrow B$ by $\psi\left(T_{i}\right)=b_{i}, 1 \leq i \leq n$. We obtain a commutative diagram (1.2). Since $D$ is defined over $C$ by the single equation $\sum_{i=1}^{n} a_{i} T_{i}-1=0$, we have $H_{D / C}=\left(a_{1}, \ldots, a_{n}\right) D=D=H_{C / A} D$; in particular, $D$ is smooth over $C$. Also, for any prime $P$ of $D$, the ideal $P \cap C$ does not contain $\left(a_{1}, \ldots, a_{n}\right)=H_{C / A}$, hence $C_{P \cap C}$ is smooth over $A$, and thus $D_{P}$ is smooth over $A$ by the transitivity of smoothness [10, Chapter 11, (28.E), p. 201]. Thus $D$ is smooth over $A$ and the desired diagram (1.2) is constructed.

Thus, to prove Theorem 1.1 and Theorem 1.2 it is sufficient to construct a diagram (1.2), with $D$ an $A$-algebra of finite type, such that $H_{D / A} B=B$. Therefore we may assume that $H_{C / A} B \varsubsetneqq B$ in (1.1). Let $P$ be a minimal prime of $H_{C / A} B$. The second observation is that to prove Theorem 1.1, it is sufficient to prove the following theorem.

Theorem 1.5. There exists a diagram (1.2) such that:

(1) $\sqrt{H_{C / A} B} \subset \sqrt{H_{D / A} B}$.

(2) $H_{D / A} B \not \subset P$.

Indeed, suppose Theorem 1.5 is known. Since $H_{C / A} B \subset P$ by definition of $P$, (1) and (2) of Theorem 1.5 imply that $\sqrt{H_{C / A} B} \varsubsetneqq \sqrt{H_{D / A} B}$. Apply Theorem 1.5 to the $A$-algebra $D$ instead of $C$, and iterate the procedure. By noetherian induction on $\sqrt{H_{C / A} B}$, after finitely many steps we will arrive at the situation when $H_{D / A} B=B$, and Theorem 1.1 will follow from Lemma 1.4.

In this paper, the $A$-algebra $D$ constructed in Theorem 1.5 will satisfy the additional condition

$$
\sqrt{H_{C / A} B} \subset \sqrt{H_{D / C} B}
$$

(in fact, (1.3) implies (1) of Theorem 1.5 by Property 2.16 below). Using this stronger version of Theorem 1.5 in the above noetherian induction argument yields Theorem 1.2.

Our original idea for the proof of Theorem 1.1 came from Lazard's theorem (recalled in $\S 3$ ), which says that an $A$-module is flat if and only if it is a filtered inductive limit of free finitely generated $A$-modules, and the realization that Theorem 1.1 is for $A$-algebras what Lazard's theorem is for $A$-modules (the analogy between regular homomorphisms $A \rightarrow B$ of rings and flat $A$-modules is discussed in more detail in $\S 2$ ). In fact, this is more than an analogy: Lazard's theorem is used in a crucial way in the proof of Theorems 1.5 and 1.2. Namely, in $\S 3$ we use 
Lazard's theorem to deduce Theorem 1.2 and Theorem 1.5 in the case when $C$ is defined over $A$ by linear homogeneous equations by writing $C=S_{A} M$, with $M$ a finite $A$-module (Proposition 3.4). Since $B$ is $A$-flat, the existence of a diagram (1.2) is given by Lazard's theorem. Indeed, consider a presentation

$$
L_{1} \stackrel{d_{1}}{\longrightarrow} L_{0} \stackrel{d_{0}}{\longrightarrow} M \rightarrow 0
$$

of $M$, where the $L_{i}$ are free of finite rank. Let $d_{1}^{*}: L_{0}^{*} \rightarrow L_{1}^{*}$ be the dual of $d_{1}$. Take a free $A$-module $K$ which maps surjectively onto $\operatorname{Ker}\left(d_{1}^{*}\right)$. We get an exact sequence

$$
K \stackrel{\alpha}{\longrightarrow} L_{0}^{*} \stackrel{d_{1}^{*}}{\longrightarrow} L_{1}^{*}
$$

Since $B$ is flat over $A$, the sequence $K \otimes_{A} B \stackrel{\alpha \otimes B}{\longrightarrow} L_{0}^{*} \otimes_{A} B \stackrel{d_{1}^{*} \otimes B}{\longrightarrow} L_{1}^{*} \otimes B$ obtained by tensoring (1.5) with $B$, is also exact. Let $u \in L_{0}^{*} \otimes B$ be the element corresponding to $\rho \circ d_{0}$ under the identification $L_{0}^{*} \otimes B \cong \operatorname{Hom}_{A}\left(L_{0}, B\right)$. We have $u \in \operatorname{Ker}\left(d_{1}^{*} \otimes B\right)$, hence

$$
u \in \operatorname{Im}(\alpha \otimes B) \text {. }
$$

To construct a diagram (1.2) for the given $A$-algebra $C$, we need flatness of $B$ over $A$ only to conclude (1.6). Thus (1.2) can be constructed even when $B$ is not flat over $A$, provided (1.6) holds. We prove Lazard's theorem and Proposition 3.4 in this slightly stronger form: in $§ \S 8-9$ we will apply it in a situation when $B$ is not necessarily flat over $A$. We note that the diagram (1.2) which we construct satisfies the condition (1.3).

With a view to Theorem 1.5, we also prove the following version of the linear homogeneous case (Proposition 3.6). Write $C=\frac{A[u]}{(f)}, f=\left(f_{1}, \ldots, f_{r}\right)$. Let $m$ be a prime ideal of $A$ such that $m A_{m} \subset \sqrt{\Delta_{f}^{0} A_{m}}$. Then there exists a diagram (1.2) such that $D \otimes_{A} A_{m}$ is smooth over $A$ and $m D \subset H_{D / C}$ (in other words, we can resolve the singularities at $m$ by a map $C \rightarrow D$ which is smooth away from $V(m)$ ). Finally, we push Proposition 3.6 even further to prove the following linearized case of Theorem 1.5. $\S \S 4-9$ are spent reducing the general case of Theorem 1.5 to the linearized one, thus proving Theorem 1.5 in its full generality.

Proposition 1.6 (the linearized case of Theorem 1.5). Consider a diagram (1.1). Assume that there exists a commutative diagram

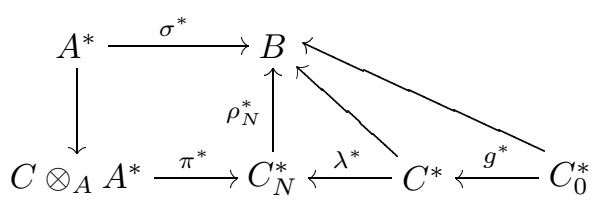

compatible with (1.1), where:

(1) $A^{*}$ is an A-algebra essentially of finite type, satisfying

$$
P \varsubsetneqq \sqrt{H_{A^{*} / A} B}
$$

(2) Let $m^{*}=P \cap A^{*}$. Then

$$
\sqrt{m^{*} B}=P
$$




$$
P \subset \sqrt{H_{C_{N}^{*} /\left(C \otimes_{A} A^{*}\right)} B} .
$$

(4) Let $m=P \cap A$ and $I=K e r \lambda^{*}$. There is a positive integer $N$ such that:

$$
\begin{aligned}
& I_{P \cap C^{*}} \subset\left(m^{*}\right)^{N} C_{P \cap C^{*}}^{*}, \\
& m^{*} C_{P \cap C^{*}}^{*} \subset \sqrt{\left(I^{2}: I\right) C_{P \cap C^{*}}^{*}} \quad \text { and } \\
& A n n_{A_{m}}\left(m^{\infty} A_{m}\right) \cap m^{N} A_{m}=(0) .
\end{aligned}
$$

(5) $C_{0}^{*}$ is defined over $A^{*}$ by linear homogeneous equations; write $C_{0}^{*}=S_{A^{*}} M=$ $\frac{A^{*}[u]}{(f)}, f=\left(f_{1}, \ldots, f_{r}\right)$.

(6) Condition (1.6) (with $A$ replaced by $A^{*}$ ) holds for $M$.

(7) $m^{*} A_{m^{*}}^{*} \subset \sqrt{\Delta_{f}^{0} A_{m^{*}}^{*}}$.

$$
H_{C^{*} / C_{0}^{*}} \not \subset P \cap C^{*} .
$$

Then the conclusion of Theorem 1.5 and (1.3) hold.

Proposition 1.6 is proved by applying Proposition 3.6 to the $A^{*}$-algebra $C_{0}^{*}$. We obtain a $C_{0}^{*}$-algebra $D_{0}$ mapping to $B$, such that

$$
\begin{aligned}
& H_{D_{0} / A^{*}} \cap A^{*} \not \subset m^{*} \text { and } \\
& m^{*} D_{0} \subset H_{D_{0} / C_{0}^{*}} .
\end{aligned}
$$

Put $D:=D_{0} \otimes_{C_{0}^{*}} C_{N}^{*}$. The algebra $D$ maps to $B$; this gives a diagram (1.2). We show that (1.8), (1.11)-(1.14) and (1.15) imply that $D_{P \cap D}$ is smooth over $A$; this gives (2) of Theorem 1.5. Moreover, from (1.8), (1.10), (1.16) and transitivity of smoothness, we get (1.3) and hence Theorem 1.5 (1).

Note that if $B$ is local with maximal ideal $P$, then, localizing by any element $x \in H_{D / A} \backslash(P \cap D)$, we get that $D_{x}$ is smooth over $A$, proving Theorem 1.2.

To prove Theorem 1.5 from Proposition 1.6, it remains to construct a diagram (1.7) satisfying conditions (1)-(8). This is accomplished in $\S \S 4-9$, first under some additional assumptions about $A$ and $B$ and then in full generality. We now outline the construction of (1.7) in $\S \S 4-9$.

Definition 1.7. Let $C$ be an $A$-algebra of finite type, $M$ a prime ideal of $C$ and $m=M \cap A$. We say that $C$ is an almost complete intersection over $A$ at $M$ if the following conditions hold:

(1) $m C_{M} \subset H_{C_{M} / A}$.

(2) There is a presentation $C=\frac{A[u]}{I}$ such that the restriction of $\frac{\mathcal{I}}{\mathcal{I}^{2}}$ to $\operatorname{Spec} C_{M} \backslash$ $V\left(m C_{M}\right)$ is a trivial vector bundle (here $\mathcal{I}$ denotes the coherent ideal sheaf on Spec $C$, given by $I$ ).

(3) Let $r=\left.\mathrm{rk} \frac{\mathcal{I}}{\mathcal{I}^{2}}\right|_{\operatorname{Spec} C_{M} \backslash V\left(m C_{M}\right)}$. Then there exist $f_{1}, \ldots, f_{r} \in I$ whose natural images in $\Gamma$ (Spec $\left.C_{M} \backslash V\left(m C_{M}\right), \frac{\mathcal{I}}{\mathcal{I}^{2}}\right)$ generate $\left.\frac{\mathcal{I}}{\mathcal{I}^{2}}\right|_{\text {Spec } C_{M} \backslash V\left(m C_{M}\right)}$.

Note that (1)-(3) of Definition 1.7 are equivalent to saying that there exist $f_{1}, \ldots, f_{r} \in I$ such that

$$
\begin{array}{ll}
m C_{M} \subset \sqrt{\Delta_{f} C_{M}} & \text { and } \\
m C_{M} & \subset \sqrt{\left.\left(I^{2}+(f)\right): I\right) C_{M}},
\end{array}
$$


where $f=\left(f_{1}, \ldots, f_{r}\right)$ (see the definition of $H_{C / A}$ and Remark 2.15 below). Note also that there are two special situations in which $C$ is an almost complete intersection over $A$ : one is when $\left.\frac{\mathcal{I}}{\mathcal{I}^{2}}\right|_{\text {Spec } C_{M} \backslash V\left(m C_{M}\right)}=0$ and Definition 1.7 (1) holds, the other when $V\left(m C_{M}\right)=\operatorname{Spec} C_{M}$, that is, when $m$ is nilpotent. In both of these cases, we will take $r=0$ and $f=\emptyset$.

In $\S 4$, we construct the diagram (1.7) (proving Theorem 1.5 and Theorem 1.2) in the following basic case. Let $P$ be a prime ideal of $B$ and let $m=P \cap A$.

Proposition 1.8. Assume that:

(1) $B$ is a local ring with maximal ideal $P$.

(2) The map $\sigma: A \rightarrow B$ induces an isomorphism $\kappa(m) \stackrel{\sim}{\rightarrow} \kappa(P)$.

(3) $C=\frac{A[u]}{I}$ is an almost complete intersection over $A$ at $P \cap C$.

(4) $\sigma$ is flat and $m B=P$.

Then there exists a diagram (1.7), satisfying conditions (1)-(8) of Proposition 1.6; in particular, Theorem 1.2 holds.

We also show (Remark 4.6) that Theorem 1.2 holds whenever assumptions (1)(3) of Proposition 1.8 are satisfied and $\sigma$ is formally smooth in the $P$-adic topology (this condition is weaker than $\sigma$ being regular - see $\S 2$ fore more details).

Our main technique here is a transformation $C \rightarrow C_{1}$, with $C_{1}$ finitely generated over $C$, called "generalized blowing up" along an ideal $M \subset A$ (Definition 4.1). Generalized blowing up of $C$ along $M$ depends on the presentation $C=\frac{A\left[u_{1}, \ldots, u_{n}\right]}{I}$ and the choice of generators of $M$, and is defined for any diagram (1.1) such that

$$
\rho\left(u_{i}\right) \in \sigma(A)+M B, 1 \leq i \leq n .
$$

For the purposes of Theorem 1.2 and conditions (1.3) and (1.10), we note that $\sqrt{M C_{1}} \subset H_{C_{1} / C}$ by definition of generalized blowing up, and that all the generalized blowings up in this paper will be along ideals $M$ such that $H_{C / A} B \subset \sqrt{M B}$. We prove Proposition 1.8 by constructing a diagram (1.7) satisfying conditions (1)(8) of Proposition 1.6, such that $A^{*}=A_{m}$ and $\pi^{*}: C \otimes_{A} A^{*} \rightarrow C_{N}^{*}=\frac{A^{*}\left[u^{(N)}\right]}{I_{N}}$ is a sequence of generalized blowings up along $m^{*} \equiv m A_{m}$. In the main part of the proof of Proposition 1.8 (Lemma 4.4) we replace (4) by a slightly more general hypothesis, in order to apply the result in $\$ \S 8-9$ in a situation where $B$ will not be flat over $A$. $\$ \S 5-9$ are devoted to gradually extending the construction of the diagram (1.7) from the basic case to the general one. In $\S 5$ we consider a diagram (1.1), such that $(A, m)$ and $(B, P)$ are local,

$$
\begin{array}{ll}
\frac{A}{m} \cong \frac{B}{P}, & \\
m B & =P \\
P & =\sqrt{H_{C / A} B .}
\end{array}
$$

We show, under some additional assumptions, more general than flatness of $B$ over $A$ (Lemma 5.4), that after a sequence $C \rightarrow C_{L}$ of generalized blowings up, $C_{L}$ is an almost complete intersection over $A$ at $P \cap C$. Combined with Proposition 1.8, this yields a diagram (1.7), satisfying (1)-(8) of Proposition 1.6. This proves Theorems 1.2 and 1.5 assuming that $B$ is flat over $A$ and conditions (1.20) 
hold. In $\S \S 6-7$, in order to achieve (3) of Proposition 1.8, we prove the following version of the Nica-Popescu theorem (see Corollary 7.9 for the original NicaPopescu theorem). Any formally smooth local homomorphism $\sigma:(A, m, k) \rightarrow$ $(B, P, K)$ of local noetherian rings, with $B$ complete, has a factorization $(A, m, k) \rightarrow$ $\left(A^{\bullet}, m^{\bullet}, K\right) \stackrel{\sigma^{\bullet}}{\longrightarrow}(B, P, K)$ such that $A^{\bullet}$ is a local noetherian ring, smooth over $A, \operatorname{dim} A^{\bullet}=\operatorname{dim} A+\operatorname{dim}_{K} H_{1}(k, K, K)$, the $\operatorname{ring}(B, P)$ is formally smooth over $\left(A^{\bullet}, m^{\bullet}\right)$ and $\sigma^{\bullet}$ induces an isomorphism of residue fields. By construction, $A^{\bullet}$ will be a filtered inductive limit of smooth finite type $A$-algebras. Furthermore, adjoin$\operatorname{ing} \operatorname{dim} B-\operatorname{dim} A^{\bullet}$ independent variables to $A^{\bullet}$, mapping them to elements of $B$ which induce a regular system of parameters of $\frac{B}{m^{\bullet} B}$ and localizing, we obtain a local noetherian ring $\left(A^{\prime}, m^{\prime}, K\right)$, such that $A^{\prime}$ is a filtered inductive limit of smooth finite type $A$-algebras, $(B, P)$ is formally smooth over $\left(A^{\prime}, m^{\prime}\right)$ and $m^{\prime} B=P$. The main interest and the main difficulty of the Nica-Popescu theorem is the case when $K$ is not separable over $k$. Incidentally, this is the only step in the proof of Theorems 1.5 and 1.2 which uses the fact that the homomorphism $\sigma$ is regular; only flatness of $B$ over $A$ is used in $\S \S 4-5$.

Now let $\hat{B}$ denote the $P$-adic completion of $B_{P}$ and let $m=P \cap A$. Applying the results of $\S 7$ to the local homomorphism $A_{m} \rightarrow \hat{B}$, we obtain a factorization $A_{m} \rightarrow A^{\prime} \rightarrow \hat{B}$ as above. Applying the results of $\S \S 4-5$ to $C \otimes_{A} A^{\prime}$ and then replacing $A^{\prime}$ by a suitable $A$-subalgebra $A_{1} \subset A^{\prime}$, smooth of finite type over $A$, we obtain the diagram

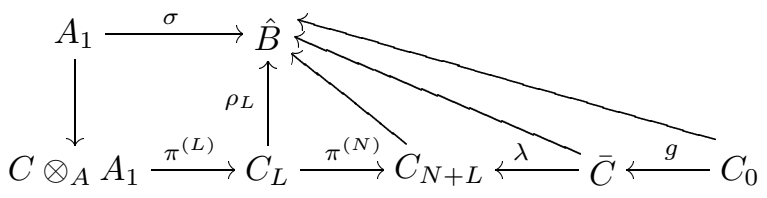

satisfying (1)-(8) of Proposition 1.6. Now Proposition 1.6 yields Theorem 1.5 and Theorem 1.2 in the case when $B$ is a complete local ring with maximal ideal $P$. In $\S 8$ we $P$-adically approximate (1.21) and obtain the diagram

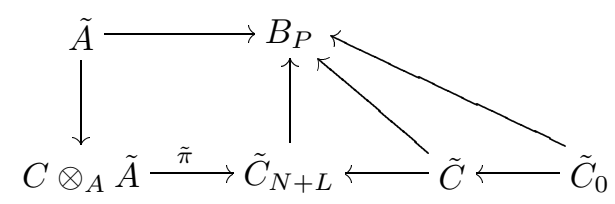

Here $B_{P}$ is not necessarily flat over $\tilde{A}$. The key point is to take a $P$-adic approximation close enough so that

(1) the hypotheses of Lemmas 4.4 and 5.4 hold, so that these lemmas still apply.

(2) (1.6) still holds for $\tilde{C}_{0}$, so that Proposition 1.6 can be applied.

Applying Proposition 1.6 to the diagram (1.22) proves Theorem 1.5 in the case when $B$ is local with maximal ideal $P$. Finally, in $\S 9$ we lift $(1.22)$ from $B_{P}$ to $B$ and obtain a diagram (1.7) satisfying (1)-(8) of Proposition 1.6. This proves Theorem 1.5, (1.3) and thus Theorem 1.2 in its full generality. The lifting of the diagram (1.22) to (1.7) will be referred to as "delocalization". In $\S 2$ we recall some basic definitions and facts about smoothness, regularity, the Jacobian ideal, André homology and the relationships between them. We use Swan's definition of the first André homology and cohomology modules. André homology appears here for two reasons. First, it is one of the main ingredients in the proof of the Nica-Popescu 
theorem: it provides a language ideally suited for measuring the inseparability of residue field extensions, induced by regular homomorphisms. The second reason is motivational: the characterization of smoothness and regularity by the vanishing of André cohomology and homology, respectively, helps clarify the analogy between $A$ algebras and $A$-modules (particularly, between regular homomorphisms $A \rightarrow B$ and flat $A$-modules $M)$. From this point of view, the André homology and cohomology modules, $H_{i}(A, B, W)$ and $H^{i}(A, B, W)$ can be viewed as the algebra analogues of $\operatorname{Tor}_{i}^{A}(M, W)$ and $\operatorname{Ext}_{A}^{i}(M, W)$, respectively.

Our motivation for considering generalized blowings up comes from the theory of resolution of singularities. Namely, consider a diagram (1.1) where $A$ is a field, $C$ a finitely generated $A$-algebra without zero divisors and $B=R_{\nu}$ a valuation ring of the field of fractions of $C$. Then the problem of constructing a diagram (1.2) (with $\psi$ injective) is nothing but the problem of local uniformization of Spec $C$ with respect to the valuation $\nu$. Of course, the valuation ring $R_{\nu}$ is not, in general, noetherian. The intersection between the problems of Local Uniformization and Néron desingularization is precisely the case when $B$ is a discrete valuation ring. In that case, Néron solved the problem by successively blowing up along the Jacobian ideal. In that sense, our proof can be regarded as a generalization of Néron's.

I would like to thank Michael Artin, Heisuke Hironaka, David Kazhdan, Arnfinn Laudal, Pierre Milman, Cristel Rotthaus, Bernard Teissier, Angelo Vistoli and Sylvia Wiegand for inspiring discussions. I thank Zach Robinson, Cristel Rotthaus and all the referees for pointing out mistakes in the earlier versions of this paper. I owe special thanks to Bernard Teissier for his careful reading of the manuscript and his extremely detailed and constructive criticism.

\section{$\S 2$. Formal smoothness, Smoothness, Jacobian CRITERION AND ANDRÉ HOMOLOGY}

In this section we recall some basic properties of formal smoothness, smoothness, the Jacobian ideal and the first André homology and cohomology, used in the rest of the paper.

André homology. Let $\sigma: A \rightarrow B$ be a homomorphism of rings and $W$ a $B$ module. Associated to this data, André [2] defines the homology and cohomology modules $H_{i}(A, B, W)$ and $H^{i}(A, B, W), i \in \mathbb{N}_{0}$, which can be viewed as obstructions to regularity and smoothness of $\sigma$, respectively (see Properties 2.4 and 2.8 below). The definitions of André homology and cohomology, given in [2], are rather technical. For the reader's convenience, we recall Swan's ad hoc definitions of $H_{i}(A, B, W)$ and $H^{i}(A, B, W), i \in\{0,1\}$ (see Definition 2.1 below and [18, §3]), which are sufficient for our purposes. It is not too hard to prove from scratch all the facts about André homology needed in this paper, using these definitions. However, we will refrain from doing so to save space, instead giving references to André [2] and Swan [18]. The readers who are already familiar with André homology will recognize that Swan's ad hoc definitions are equivalent to those of André. We now define $H_{i}(A, B, W)$ and $H^{i}(A, B, W)$ for $i \in\{0,1\}$, following Swan $[18, \S 3]$. Take an exact sequence

$$
0 \rightarrow I \rightarrow F \stackrel{\pi}{\rightarrow} B \rightarrow 0
$$


where $F$ is a localization of a free $A$-algebra and $I$ is a (not necessarily finitely generated) ideal of $F$. (2.1) gives rise to the exact sequence

$$
\frac{I}{I^{2}} \stackrel{d}{\rightarrow} \Omega_{F / A} \otimes_{F} B \rightarrow \Omega_{B / A} \rightarrow 0
$$

(the second fundamental exact sequence for Kähler differentials [10, Chapter 10, (26.I), Theorem 58, p. 187]). Tensoring (2.2) with $W$ over $B$ gives the exact sequence

$$
\frac{I}{I^{2}} \otimes_{B} W \stackrel{d \otimes W}{\longrightarrow} \Omega_{F / A} \otimes_{F} W \rightarrow \Omega_{B / A} \otimes_{B} W \rightarrow 0 .
$$

Taking $B$-homomorphisms into $W$ in (2.2), we obtain the exact sequence

$$
\operatorname{Hom}_{B}\left(\frac{I}{I^{2}}, W\right) \overleftarrow{\partial_{W}} \operatorname{Der}_{A}(F, W) \leftarrow \operatorname{Der}_{A}(B, W) \leftarrow 0 .
$$

Note that $\Omega_{F / A} \otimes_{F} B$ is a free $B$-module and $\operatorname{Der}_{A}(F, B)$ is free whenever $F$ is finitely generated over $B$.

Definition 2.1 (Swan $[18, \S 3]$ ). André homology modules and cohomology modules, $H_{i}(A, B, W)$ and $H^{i}(A, B, W)$ for $i \in\{0,1\}$, are defined as follows:

$$
\begin{aligned}
& H_{0}(A, B, W)=\Omega_{B / A} \otimes_{B} W \equiv \operatorname{Coker}(d \otimes W), \\
& H_{1}(A, B, W)=\operatorname{Ker}(d \otimes W), \\
& H^{0}(A, B, W)=\operatorname{Der}_{A}(B, W) \equiv \operatorname{Ker} \partial_{W}, \\
& H^{1}(A, B, W)=\text { Coker } \partial_{W} .
\end{aligned}
$$

It is immediate to show that for $i \in\{0,1\}, H_{i}(A, B, W)$ and $H^{i}(A, B, W)$ are independent of the presentation (2.1), and also that $H_{i}(A, \cdot, W)$ and $H^{i}(A, \cdot, W)$ are, respectively, a covariant and a contravariant functor of $B$ (see [18, Part II, Lemma 3.1]).

Property 2.2. Let $\sigma: A \rightarrow B$ be a surjective ring homomorphism, let $I=$ Ker $\sigma$ and let $W$ be a $B$-module. We have $H_{0}(A, B, W)=H^{0}(A, B, W)=0$ and $H_{1}(A, B, W) \cong \frac{I}{I^{2}} \otimes_{B} W$.

Proof. The exact sequence $0 \rightarrow I \rightarrow A \stackrel{\sigma}{\rightarrow} B \rightarrow 0$ gives a presentation of $B$ as an $A$-module. Since $\Omega_{A / A}=0$, the result follows immediately from definitions.

Property 2.3 (the Jacobi-Zariski sequence). Let $F \rightarrow B$ be a homomorphism of $A$-algebras and let $W$ be a $B$-module. There are two natural exact sequences:

$$
\begin{aligned}
H_{1}(A, F, W) \rightarrow H_{1}(A, B, W) & \rightarrow H_{1}(F, B, W) \rightarrow H_{0}(A, F, W) \\
& \rightarrow H_{0}(A, B, W) \rightarrow H_{0}(F, B, W) \rightarrow 0
\end{aligned}
$$

and

$$
\begin{aligned}
0 \rightarrow H^{0}(F, B, W) & \rightarrow H^{0}(A, B, W) \rightarrow H^{0}(A, F, W) \\
& \rightarrow H^{1}(F, B, W) \rightarrow H^{1}(A, B, W) \rightarrow H^{1}(A, F, W) .
\end{aligned}
$$

Proof. See [18, Part II, Theorem 3.3]. Again, Swan only proves the Jacobi-Zariski sequence for homology with coefficients in $B$. To get (2.5), tensor everything in Swan's diagram $\left(^{*}\right)$ with $W$; to get (2.6), take homomorphisms into $W$. 
Homological characterizations of smoothness and regularity. Let $\sigma: A \rightarrow$ $B$ be a homomorphism of rings.

Property 2.4. Consider the following conditions:

(1) B is smooth over $A$.

(2) The sequence

$$
0 \rightarrow \frac{I}{I^{2}} \stackrel{d}{\rightarrow} \Omega_{F / A} \otimes_{F} B \rightarrow \Omega_{B / A} \rightarrow 0
$$

is split exact (this condition is sometimes expressed by saying that the homomorphism $d$ has a left inverse).

(3) $H_{1}(A, B, B)=0$ and $\Omega_{B / A}$ is a projective $B$-module.

(4) $H^{1}(A, B, W)=0$ for all $B$-modules $W$.

(5) $H_{1}(A, B, B)=0$ and $\Omega_{B / A}$ is a flat $B$-module.

(6) $H_{1}(A, B, W)=0$ for all $B$-modules $W$.

We have the implications: (1) $\Longleftrightarrow(2) \Longleftrightarrow$ (3) $\Longleftrightarrow(4) \Longrightarrow(5) \Longleftrightarrow(6)$. Suppose, in addition, that either $\frac{I}{I^{2}}$ or $\Omega_{B / A}$ is a finitely generated $B$-module. Then we also have (5) $\Longrightarrow(3)$, so that all the conditions (1)-(6) are equivalent. Finally, $B$ is étale over $A$ if and only if $H_{1}(A, B, B)=\Omega_{B / A}=0$.

Proof. The implications $(3) \Longleftrightarrow(4) \Longrightarrow(5) \Longleftrightarrow(6)$, as well as the equivalence $(3) \Longleftrightarrow(5)$ in case $\frac{I}{I^{2}}$ or $\Omega_{B / A}$ is a finitely generated $B$-module, are immediate from definitions; for the rest, see [18, Theorem 3.4].

Note that (1), (3) and (4) of Property 2.4 do not depend on the choice of the presentation (2.1). Thus, in particular, Property 2.4 says that if (2) holds for one presentation (2.1), then it also holds for any other presentation.

Corollary 2.5. Let $\sigma: A \rightarrow B$ be a homomorphism of rings. Then $B$ is smooth over $A$ if and only if $B_{P}$ is smooth over $A$ for every prime ideal $P$ of $B$.

Proof. Use the notation of (2.1). For a prime ideal $P$ of $B, 0 \rightarrow I_{P \cap F} \rightarrow F_{P \cap F} \rightarrow$ $B_{P}$ is a presentation of $B_{P}$. The sequence (2.7) is split exact if and only if it is split exact after tensoring with $B_{P}$ for all $P \in \operatorname{Spec} B$, and the result follows.

Property 2.4 says that acyclicity in cohomology characterizes smoothness. On the other hand, conditions (5) and (6) - acyclycity in homology - characterize regular homomorphisms, as we now explain. We start with the case when $A$ is a field.

Proposition 2.6. ([8, EGA $\left.0_{\mathrm{IV}}(22.5 .8)\right]$, [10, (39.C), Theorem 93] and [2, Lemma III.21, Corollary VII.27 and Proposition XVI.17]) Let $(A, m, k) \rightarrow(B, M, K)$ be $a$ local homomorphism of local noetherian rings. The following conditions are equivalent:

(1) $H_{1}(A, B, K)=0$.

(2) $B$ is formally smooth over $A$ in the $M$-adic topology.

If $A$ is a field, then (1) and (2) are also equivalent to saying that $B$ is geometrically regular over $k$.

Proposition 2.7. (Grothendieck [8, Theorem (19.7.1)], [2, Proposition XV.19, p. 211]) Let $(A, m, k) \rightarrow(B, P, K)$ be a local homomorphism of local noetherian rings. The following conditions are equivalent: 
(1) $B$ is formally smooth over $A$ in the P-adic topology.

(2) $B$ is flat over $A$ and $\frac{B}{m B}$ is geometrically regular over $k$.

Property 2.8 (André's theorem [2, Theorem 30, p. 331]). Let $\sigma: A \rightarrow B$ be a homomorphism of noetherian rings. Then the following conditions are equivalent:

(1) $\sigma$ is regular.

(2) (6) of Property 2.4 holds.

(3) $H_{1}(A, B, \kappa(P))=0$ for any prime ideal $P$ of $B$.

(4) For every prime ideal $P \subset B, B_{P}$ is formally smooth over $A$ with respect to the P-adic topology.

There are two situations in which acyclicity in homology and cohomology are the same thing:

Corollary 2.9. Let $\sigma: A \rightarrow B$ be a homomorphism of noetherian rings. Assume that either $B$ is a field or $B$ is essentially of finite type over $A$. Then $B$ is smooth over $A$ if and only if $\sigma$ is regular.

Proof. If $B$ is a field, then $\Omega_{B / A}$ is a $B$-vector space. If $B$ is essentially of finite type over $A$, then $\Omega_{B / A}$ is a finite $B$-module. In either case, $\Omega_{B / A}$ is projective if and only if it is flat. Now the corollary follows from Properties 2.4 and 2.8.

Remark 2.10. Let $A_{1} \stackrel{\sigma_{1}}{\longrightarrow} A_{2} \stackrel{\sigma_{2}}{\longrightarrow} A_{3}$ be ring homomorphisms and $W$ an $A_{3}$-module such that $H_{1}\left(A_{1}, A_{3}, W\right)=\Omega_{A_{2} / A_{1}} \otimes_{A_{2}} W=0$. Then $H_{1}\left(A_{2}, A_{3}, W\right)=0$ (this follows immediately from the Jacobi-Zariski sequence).

Assume that $A_{1}, A_{2}$ and $A_{3}$ are noetherian, that $\sigma_{2} \circ \sigma_{1}$ is a regular homomorpism and that $\Omega_{A_{2} / A_{1}}=0$. Then $\sigma_{2}$ is a regular homomorpism (this follows from the above and the equivalence of (1)-(3) of Property 2.8).

The Jacobian criterion. Let $A$ be a ring and $C$ an $A$-algebra, essentially of finite type over $A$. Fix a presentation

$$
C=\frac{A\left[u_{1}, \ldots, u_{n}\right]_{S}}{I},
$$

where $I$ is a finitely generated ideal of $A\left[u_{1}, \ldots, u_{n}\right]$ and $S$ a multiplicative subset of $A\left[u_{1}, \ldots, u_{n}\right]$, disjoint from $I$. Choose a base $f=\left(f_{1}, \ldots, f_{l}\right)$ of $I$.

Definition 2.11 (Elkik [7] and H. Hironaka). The Jacobian ideal of $C$ over $A$, denoted $H_{C / A}$, is the ideal $H_{C / A}:=\sqrt{\sum_{g} \Delta_{g}((g): I) C}$, where $g$ ranges over all the subsets of $\left\{f_{1}, \ldots, f_{l}\right\}$.

Remark 2.12. Apparently, the definition of $H_{C / A}$ depends on the presentation (2.8) and on the choice of a base for $I$. Property 2.13 below says that $H_{C / A}$ is the defining ideal of the non-smooth locus of $C$ over $A$ and therefore depends only on $C$ itself, not on the particular presentation nor on the choice of $f$.

Property 2.13. The ideal $H_{C / A}$ defines the non-smooth locus of $C$ over $A$. In other words, for a prime ideal of $P \subset C, C_{P}$ is smooth over $A$ if and only if $H_{C / A} \not \subset P$. We have

$$
H_{C / A}=\left\{x \in C \mid C_{x} \text { is smooth over } A\right\}
$$

(remember that the zero ring is smooth over anything!). In particular, $C$ is smooth over $A$ if and only if $H_{C / A}=C$. 
Proof. Let $F=A\left[u_{1}, \ldots, u_{n}\right]_{S}$ in (2.8). We have $\Omega_{F / A} \cong \bigoplus_{i=1}^{n} F d u_{i}$ and $\Omega_{F / A} \otimes_{F} C \cong$ $\bigoplus_{i=1}^{n} C d u_{i}$. First, suppose that $C$ is local with maximal ideal $P$ and residue field $K$.

Lemma 2.14. $C$ is smooth over $A$ if and only if there exist $g_{1}, \ldots, g_{r} \in\left\{f_{1}, \ldots, f_{l}\right\}$ as above such that $I=\left(g_{1}, \ldots, g_{r}\right)$ and $\Delta_{g} C=C$.

Proof. By Property $2.4(1) \Longleftrightarrow(2), C$ is smooth over $A$ if and only if the sequence

$$
0 \rightarrow \frac{I}{I^{2}} \stackrel{d}{\rightarrow} \bigoplus_{i=1}^{n} C d u_{i} \rightarrow \Omega_{C / A} \rightarrow 0
$$

is split exact. Since $C$ is local, (2.10) is split exact if and only if it is a split exact sequence of free modules. This happens if and only if there is a subset $g=\left\{g_{1}, \ldots, g_{r}\right\} \subset\left\{f_{1}, \ldots, f_{l}\right\}$ which freely generate $\frac{I}{I^{2}}$ and such that $d g_{1}, \ldots, d g_{r}$ are $K$-linearly independent modulo $P$. The latter condition says precisely that the matrix $\left(\frac{\partial g_{i}}{\partial u_{j}}\right)_{\substack{1 \leq i \leq r \\ 1 \leq j \leq n}}$ has rank $r$ modulo $P$, that is, $\Delta_{g} C=C$. Now, if such a subset $g$ exists, then $g_{1}, \ldots, g_{r}$ generate $I$ by Nakayama's lemma. Conversely, if there is a subset $g=\left\{g_{1}, \ldots, g_{r}\right\} \subset\left\{f_{1}, \ldots, f_{l}\right\}$ such that $\left(\frac{\partial g_{i}}{\partial u_{j}}\right)_{\substack{1 \leq i \leq r \\ 1 \leq j \leq n}}$ has rank $r$ modulo $P$ and $\left(g_{1}, \ldots, g_{r}\right)=I$, then it is immediate to check that the images of $g_{1}, \ldots, g_{r}$ freely generate $\frac{I}{I^{2}}$. This completes the proof.

Now drop the hypothesis that $C$ is local. Since we have not yet proved that $H_{C / A}$ is independent of presentation, we will provisionally talk about $H_{C / A}$ with respect to the given presentation. Because we are assuming that $I$ is finitely generated, the operation : commutes with localization. Thus $H_{C / A}$ localizes well: take any multiplicative subset $S \subset C$. Let $R:=S \cap F$ and define $H_{C_{S} / A}$ using the presentation $C_{S}=\frac{F_{R}}{I F_{R}}$. We have a canonical isomorphism $\left(H_{C / A}\right)_{S} \cong H_{C_{S} / A}$. For a prime ideal $P \subset C$, apply Lemma 2.14 to the local ring $C_{P}$ (again, we use the presentation of $C_{P}$, obtained from (2.8) by localization). By Lemma 2.14, $C_{P}$ is smooth over $A$ if and only if there exists a subset $g=\left\{g_{1}, \ldots, g_{r}\right\} \subset\left\{f_{1}, \ldots, f_{l}\right\}$ such that

$$
\left(g_{1}, \ldots, g_{r}\right) F_{P \cap F}=I_{P \cap F}
$$

and

$$
\Delta_{g} \not \subset P \cap F \text {. }
$$

Now, (2.11) is equivalent to saying that $((g): I) F_{P \cap F}=F_{P \cap F}$, and also to

$$
((g): I) C_{P} \not \subset P C_{P} \text {. }
$$

Combining (2.12) and (2.13) and using the fact that both the operation of taking $\Delta_{g}$ and : commute with localization, we get that $C_{P}$ is smooth over $A$ if and only if $\Delta_{g}((g): I) C \not \subset P$ for some $g$ as above. Thus $C_{P}$ is smooth over $A$ if and only if $H_{C / A} \not \subset P$. This proves the first statement of Property 2.13.

Since $H_{C / A}$ is radical by definition, it equals the intersection of all the primes $P \subset B$ such that $B_{P}$ is not smooth over $A$. Now (2.9) follows from Corollary 2.5. The last statement of Property 2.13 follows immediately.

Note, in particular, that $H_{C / A}$ is well defined, i.e. is independent of the choice of presentation (2.8) and the generators $f_{i}$. 
Remark 2.15. In the situation of Property 2.13, assume that $F$ is noetherian. Suppose that $C_{P}$ is smooth over $A$. Let $Q$ be a minimal prime of $I$, contained in $P \cap F$. We have $I F_{Q}=Q F_{Q}$. Then (2.11) implies that $g$ is a system of parameters (even a regular system of parameters) for $F_{Q}$. This shows that when $F$ is noetherian, we may let $g$ in the definition of $H_{C / A}$ range only over those subsets $\left\{g_{1}, \ldots, g_{r}\right\}$ of $\left\{f_{1}, \ldots, f_{l}\right\}$ which form a system of parameters in $F_{Q}$, for some minimal prime $Q$ of $I$. In particular, if $C$ is a complete intersection over $A$ and $f$ is a minimal set of generators of $I$, then $H_{C / A}=\sqrt{\Delta_{f} C}$.

Consider homomorphisms $A \stackrel{\sigma}{\rightarrow} B \stackrel{\phi}{\rightarrow} C$ of noetherian rings, where $B$ is essentially of finite type over $A$ and $C$ is essentially of finite type over $B$. The following property describes the relationship between $H_{B / A}, H_{C / A}$ and $H_{B / C}$.

Property 2.16. We have $\sqrt{H_{C / B} H_{B / A} C}=\sqrt{H_{C / B} H_{C / A}}$.

Remark 2.17. Property 2.16 says that a prime ideal $P \subset C$, not containing $H_{C / B}$, contains $H_{B / A} C$ if and only if it contains $H_{C / A}$. In view of Property 2.13 , this can be restated as follows. Take any prime ideal $P \subset C$, such that $C_{P}$ is smooth over $B$. Then $C_{P}$ is smooth over $A$ if and only if $B_{P \cap B}$ is smooth over $A$.

Proof of Property 2.16. By Corollary 2.9, each of the homomorphisms $\sigma, \phi$ and $\phi \circ \sigma$ is regular if and only if it is smooth. Now Property 2.16 follows from [10, (33.B)] and Proposition 2.7 (1) $\Longrightarrow(2)$.

Field extensions. Next, we discuss some standard results, which can be interpreted as a restriction of the above theory to homomorphisms of fields instead of rings. A detailed study of extensions $k \rightarrow K$ with $\operatorname{dim}_{K} H_{1}(k, K, K)<\infty$ will be undertaken in $\S 6$. Let $k \rightarrow K$ be a field extension. Then $\Omega_{K / k}$ and $H_{1}(k, K, K)$ are $K$-vector spaces. If $K$ is finitely generated over $k$, then $\Omega_{K / k}$ and $H_{1}(k, K, K)$ are finite-dimensional.

Property 2.18 ([18, Corollary 5.2]). Let $k \rightarrow L \rightarrow K$ be homomorphisms of fields. The first map on the left in the Jacobi-Zariski sequence (2.5) is injective. In other words, we have an exact sequence

$$
\begin{aligned}
0 \rightarrow H_{1}(k, L, K) \rightarrow & H_{1}(k, K, K) \rightarrow H_{1}(L, K, K) \\
& \rightarrow \Omega_{L / k} \otimes_{L} K \rightarrow \Omega_{K / k} \rightarrow \Omega_{K / L} \rightarrow 0 .
\end{aligned}
$$

This result is stated in [18] with $H_{1}(k, L, L) \otimes_{L} K$ instead of $H_{1}(k, L, K)$. However, the two statements amount to the same thing since $H_{1}(k, L, L) \otimes_{L} K \cong$ $H_{1}(k, L, K)$. In fact, we have the same exact sequence for homology with coefficients in any $K$-vector space $W$ instead of $K$ (tensor everything with $W$ and use that $W$ is $K$-flat).

Property 2.19 ([18, Corollary 5.5]). $K$ is separable over $k \Longleftrightarrow K$ is smooth over $k \Longleftrightarrow H_{1}(k, K, K)=0 \Longleftrightarrow H_{1}(k, K, W)=0$ for any $K$-vector space $W$.

(The last two equivalences follow from Property 2.4.)

We end this section with the local criterion of flatness.

Proposition 2.20. (Local criterion of flatness, [10, (20.C), Theorem 49, p. 146] or [18, Theorem 7.1]) Let $\sigma: A \rightarrow B$ be a homomorphism of noetherian rings, $I$ an ideal of $A$ such that $I B \subset J a c(B)$ and $M$ a finitely generated $B$-module. The following conditions are equivalent: 
(1) $M$ is flat over $A$.

(2) $\frac{M}{I M}$ is flat over $\frac{A}{I}$ and

$$
\operatorname{Tor}_{1}^{A}\left(M, \frac{A}{I}\right)=0 \text {. }
$$

(3) $\frac{M}{I M}$ is flat over $\frac{A}{I}$ and for all $n \in \mathbb{N}$, the canonical map $\frac{I^{n}}{I^{n+1}} \otimes_{\frac{A}{I}} \frac{M}{I M} \rightarrow \frac{I^{n} M}{I^{n+1} M}$ is an isomorphism.

Proposition 2.21. Let $A$ be a ring, $M$ an $A$-module and $\left(x_{1}, \ldots, x_{n}\right)$ a regular sequence in $A$, which is also a regular sequence for $M$. Let $I=\left(x_{1}, \ldots, x_{n}\right)$. Then $\operatorname{Tor}_{1}^{A}\left(M, \frac{A}{I}\right)=0$.

Proof. Straightforward induction on $n$. See [18, Lemma 7.5].

Corollary 2.22. Let $(A, m, k) \stackrel{\alpha}{\longrightarrow}\left(A^{\prime}, m^{\prime}, k^{\prime}\right) \stackrel{\sigma^{\prime}}{\longrightarrow}(B, P, K)$ be local homomorphisms of local noetherian rings. Assume that:

(1) Both $A^{\prime}$ and $B$ are flat over $A$.

(2) $A_{0}^{\prime}:=\frac{A^{\prime}}{m A^{\prime}}$ and $B_{0}:=\frac{B}{m B}$ are regular local rings.

(3) There exist elements $x_{1}, \ldots, x_{a} \in A^{\prime}$ which induce a regular system of parameters of $A_{0}^{\prime}$ and whose images in $B_{0}$ can be extended to a regular system of parameters of $B_{0}$.

Then $B$ is flat over $A^{\prime}$ (hence faithfully flat, hence $\sigma^{\prime}$ is injective).

Proof. Since $B$ is flat over $A$, we have $\operatorname{Tor}_{1}^{A}(k, B)=0$. Since $A^{\prime}$ is flat over $A$, we obtain

$$
\operatorname{Tor}_{1}^{A}\left(A_{0}^{\prime}, B\right) \equiv \operatorname{Tor}_{1}^{A}\left(k \otimes_{A} A^{\prime}, B\right)=\operatorname{Tor}_{1}^{A}(k, B) \otimes_{A} A^{\prime}=0 .
$$

Let $\bar{x}:=\bar{x}_{1}, \ldots, \bar{x}_{a}$ denote the images of $x_{1}, \ldots, x_{a}$ in $A_{0}^{\prime}$. By our assumptions, $\bar{x}$ is a regular sequence both for $A_{0}^{\prime}$ and for $B_{0}$; moreover, $\frac{A_{0}^{\prime}}{(\bar{x})}$ is a field. Thus $B_{0}$ is flat over $A_{0}^{\prime}$ by the local criterion of flatness, applied at the ideal $(\bar{x}) A_{0}^{\prime}$. Combining this with (2.15) and applying the local criterion of flatness once again, this time at the ideal $m A^{\prime}$, proves that $B$ is flat over $A^{\prime}$.

Remark 2.23. Let $A \rightarrow B$ be a continuous, flat homomorphism of topological rings, with topologies defined by ideals $m \subset A$ and $P \subset B$. Let $\hat{A}$ be the $m$-adic completion of $A, \hat{B}$ the $P$-adic completion of $B$ and $\tilde{B}$ the $m B$-adic completion of $B$. Then $\tilde{B}$ is flat over $\hat{A}$ by Proposition $2.20(1) \Longleftrightarrow(3)$, and $\hat{B}$ is flat over $\tilde{B}$, being the $P \tilde{B}$-adic completion of $\tilde{B}$. Thus $\hat{B}$ is flat over $\hat{A}$.

Now let the notation and assumptions be as in Corollary 2.22 and consider the induced homomorphisms $\hat{A} \stackrel{\hat{\alpha}}{\longrightarrow} \hat{A}^{\prime} \stackrel{\hat{\sigma}^{\prime}}{\longrightarrow} \hat{B}$ between formal completions. The above considerations show that $\hat{\alpha}$ and $\hat{\sigma}^{\prime} \circ \hat{\alpha}$ are flat, and hence the new triple satisfies the assumptions of Corollary 2.22. Thus $\hat{B}$ is flat (hence faithfully flat) over $\hat{A}^{\prime}$; in particular, $\hat{\sigma}^{\prime}$ is injective.

Remark 2.24. Let $\sigma: A \rightarrow B$ be a homomorphism of topological rings, with the respective topologies defined by ideals $P \subset B$ and $m=P \cap A$. Let $\hat{A}$ denote the $m$-adic completion of $A$ and $\hat{B}$ the $P$-adic completion of $B$. Then $B$ is formally smooth over $A$ if and only if $\hat{B}$ is formally smooth over $\hat{A}$, if and only if $\frac{B}{P^{n}}$ is formally smooth over $\frac{A}{m^{n}}$ for all $n \in \mathbb{N}$. In particular, $B$ is formally smooth over 
$A$ whenever $\hat{A} \cong \hat{B}$ via the natural homomorphism induced by $\sigma$ (all of this is immediate from definitions).

Corollary 2.25. Let the assumptions be as in Corollary 2.22. Assume, in addition, that $B$ is formally smooth over $A$, that $A^{\prime}=A\left[x_{1}, \ldots, x_{a}\right]_{P \cap A\left[x_{1}, \ldots, x_{a}\right]}$ and that $(m, x) B=P$

$$
\frac{B}{P} \cong \frac{A}{m}
$$

Then $B$ is formally smooth over $A^{\prime}$.

Proof. By Remark 2.23 the induced map $\hat{\sigma}: \hat{A}[[x]] \rightarrow \hat{B}$ is injective. On the other hand, since $(m, x) B=P$ and in view of $(2.16), \hat{\sigma}$ is surjective, hence an isomorphism. The corollary follows from Remark 2.24 (this fact, even without the assumption (2.16), also follows easily from the Jacobi-Zariski sequence for the triple $A \rightarrow A^{\prime} \rightarrow B$ and the $B$-module $\left.K\right)$.

\section{§3. Smoothing of Algebras Defined By Linear homogeneous EQUATIONS}

In this section we prove Theorem 1.2 in the case when $C$ is defined over $A$ by linear homogeneous equations. For that we do not need $\sigma$ to be regular: it is sufficient to assume that $\sigma$ is flat (in fact, an even weaker hypothesis will do-see Proposition 3.4). All of this is well known and is a consequence of Lazard's theorem [10, (3.A), Theorem 1 (6), p. 18] and [5, pp. 7-8]. We reproduce these results here in order to go on and prove a stronger version of them (Proposition 1.6) which will play a central role in the rest of the paper.

Let $A$ be a ring and $\rho: M \rightarrow B$ a homomorphism of $A$-modules, with $M$ finitely generated. Consider a presentation

$$
L_{1} \stackrel{d_{1}}{\longrightarrow} L_{0} \stackrel{d_{0}}{\longrightarrow} M \rightarrow 0
$$

of $M$, where the $L_{i}$ are free and $L_{0}$ is of finite rank. Let $d_{1}^{*}: L_{0}^{*} \rightarrow L_{1}^{*}$ be the dual of $d_{1}$. Take a free $A$-module $K$ which maps surjectively onto $\operatorname{Ker}\left(d_{1}^{*}\right)$. We get an exact sequence

$$
K \stackrel{\alpha}{\longrightarrow} L_{0}^{*} \stackrel{d_{1}^{*}}{\longrightarrow} L_{1}^{*}
$$

Consider the complex

$$
K \otimes_{A} B \stackrel{\alpha \otimes B}{\longrightarrow} L_{0}^{*} \otimes_{A} B \stackrel{d_{1}^{*} \otimes B}{\longrightarrow} L_{1}^{*} \otimes B
$$

obtained by tensoring (3.2) with $B$. Let $u \in L_{0}^{*} \otimes B$ be the element corresponding to $\rho \circ d_{0}$ under the identification $L_{0}^{*} \otimes B \cong \operatorname{Hom}_{A}\left(L_{0}, B\right)$.

Remark 3.1. By construction, $u \in \operatorname{Ker}\left(d_{1}^{*} \otimes B\right)$. If $B$ is flat over $A$, then the sequence (3.3) is exact, so that $u \in \operatorname{Im}(\alpha \otimes B)$.

Proposition 3.2. Assume that $u \in \operatorname{Im}(\alpha \otimes B)$. Then there exists a factorization

$$
M \stackrel{\phi}{\longrightarrow} F \stackrel{\psi}{\longrightarrow} B
$$

of $\rho$ through a free finitely generated $A$-module $F$. If $A$ is noetherian, we can choose a factorization (3.4) with the following additional property. For any $P \in \operatorname{Spec} A$ such that $M_{P}$ is a free $A_{P}$-module, the map $\phi_{P}: M_{P} \rightarrow F_{P}$ induced by $\phi$ has a left inverse. 
Proof. Choose a free finitely generated submodule $K_{0}$ of $K$ such that $u \in$ $(\alpha \otimes B)\left(K_{0} \otimes B\right)$. Consider the complex

$$
K_{0} \stackrel{\alpha}{\longrightarrow} L_{0}^{*} \stackrel{d_{1}^{*}}{\longrightarrow} L_{1}^{*} .
$$

Put $F:=K_{0}^{*}$. Dualizing (3.5) and using the fact that $L_{1} \subset L_{1}^{* *}$, we get a complex $L_{1} \stackrel{d_{1}}{\longrightarrow} L_{0} \stackrel{\alpha *}{\longrightarrow} F$. The homomorphism $\alpha^{*}$ induces a homomorphism $\phi: M \rightarrow F$. Take an element $v \in K_{0} \otimes B$ such that $(\alpha \otimes B)(v)=u$. Let $\psi \in H o m_{A}(F, B)$ be the element corresponding to $v$ under the identification $\operatorname{Hom}_{A}(F, B) \cong K_{0} \otimes B$. Then $\psi \circ \phi=\rho$ and (3.4) is constructed. Now assume that $A$ is noetherian. Then $K$ is finitely generated, so we may take $K_{0}=K$ in (3.5). Then $F$ is finitely generated. Take a prime $P \subset A$ such that $M_{P}$ is a free $A_{P}$-module. Then $\left(d_{0}\right)_{P}:\left(L_{0}\right)_{P} \rightarrow M_{P}$ has a right inverse, i.e. $M_{P}$ is a direct summand of $\left(L_{0}\right)_{P}$. Then $M_{P}^{*}$ is a direct summand of $\left(L_{0}^{*}\right)_{P}$, so $\alpha_{P}$ induces a surjection of free modules $K_{P} \rightarrow M_{P}^{*}$. Hence $M_{P}$ is a direct summand of $F_{P}=K_{P}^{*}$, so $\phi$ has a left inverse.

Corollary 3.3 (Lazard's theorem). Let $A$ be a ring and $\rho: M \rightarrow B$ a homomorphism of $A$-modules, where $B$ is flat and $M$ finitely generated. There exists a factorization (3.4) of $\rho$ through a free finitely generated $A$-module $F$. In other words, $B$ is a filtered direct limit of free finitely generated $A$-modules. If $A$ is noetherian, we can choose (3.4) so that whenever $P \in \operatorname{Spec} A$ and $M_{P}$ is a free $A_{P}$-module, $\phi_{P}$ has a left inverse.

Proof. Immediate from Proposition 3.2 and Remark 3.1.

We pass to symmetric algebras in (3.4) to establish Theorem 1.2 in the case when $C$ is defined over $A$ by linear homogeneous equations. Let $\sigma: A \rightarrow B$ be a ring homomorphism. Consider a commutative diagram (1.1). Suppose $C$ has the form $C=\frac{A\left[u_{1}, \ldots, u_{n}\right]}{I}$, where $I=\left(f_{1}, \ldots, f_{m}\right)$ and each $f_{j}$ is a linear homogeneous equation in the $u_{i}$ :

$$
f_{j}=\sum_{i=1}^{n} a_{i j} u_{i}, \quad a_{i j} \in A
$$

Then $C=S_{A} M$, where $M$ is the $A$-module with generators $u_{1}, \ldots, u_{n}$ and relations $f_{1}, \ldots, f_{m}$. Consider a presentation (3.1) of $M$. Then $\rho$ induces an $A$-module homomorphism $\left.\rho\right|_{M}: M \rightarrow B$. Let $K$ and $u \in \operatorname{Ker}\left(d_{1}^{*} \otimes B\right)$ be as above.

Proposition 3.4. Assume that $u \in \operatorname{Im}(\alpha \otimes B)$ in (3.3) (this holds, in particular, whenever $B$ is flat over $A$ ). Then there exists a commutative diagram (1.2) where $D$ is a polynomial ring in finitely many variables over $A$. If, in addition, $A$ is noetherian, there exists a diagram (1.2) such that

$$
H_{C / A} D \subset H_{D / C}
$$

(remember that the Jacobian ideal $H_{D / C}$ is radical by definition!).

Remark 3.5. Since $D$ is a polynomial ring over $A$, we have $H_{D / A}=D$. Then by Property 2.16, (3.7) is equivalent to saying that $\sqrt{H_{C / A} D}=H_{D / C}$.

Proof of Proposition 3.4. Let

$$
M \stackrel{\phi_{1}}{\longrightarrow} F \stackrel{\psi_{1}}{\longrightarrow} B
$$


be the factorization of $\left.\rho\right|_{M}$, described in Proposition 3.2. Put $D:=S_{A} F$. (3.8) induces maps $C \stackrel{\phi}{\longrightarrow} D \stackrel{\psi}{\longrightarrow} B$ of symmetric algebras with $\rho=\psi \circ \phi$ and hence a commutative diagram (1.2). Let $P$ be a prime ideal of $D$ such that $H_{C / A} \not \subset P \cap C$. Let $Q=P \cap A$. Since $H_{C / A} \not \subset P \cap C$, there exists a subset of $\left\{f_{1}, \ldots, f_{m}\right\}$, say $\left\{f_{1}, \ldots, f_{r}\right\}$, such that $\Delta_{\left(f_{1}, \ldots, f_{r}\right)}^{0} \not \subset Q$ and $\left(\left(f_{1}, \ldots, f_{r}\right): I\right) C \not \subset P \cap C$. Then $\frac{A_{Q}\left[u_{1}, \ldots, u_{n}\right]}{\left(f_{1}, \ldots, f_{r}\right)}$ is isomorphic to a polynomial ring over $A_{Q}$, hence all of its associated primes are extended from $A_{Q}$. Since $\left(\left(f_{1}, \ldots, f_{r}\right): I\right) C \not \subset P \cap C,\left(\left(f_{1}, \ldots, f_{r}\right)\right.$ : I) $\frac{A_{Q}\left[u_{1}, \ldots, u_{n}\right]}{\left(f_{1}, \ldots, f_{r}\right)}$ cannot be contained in any proper ideal extended from $A_{Q}$. Thus $\left(\left(f_{1}, \ldots, f_{r}\right): I\right) A_{Q}\left[u_{1}, \ldots, u_{n}\right]=A_{Q}\left[u_{1}, \ldots, u_{n}\right]$, so that $C \otimes_{A} A_{Q}=\frac{A_{Q}\left[u_{1}, \ldots, u_{n}\right]}{\left(f_{1}, \ldots, f_{r}\right)}$, which implies that $M_{Q}$ is a free $A_{Q}$-module. Now assume that $A$ is noetherian. By Proposition 3.2 we may choose (3.8) so that $\left(\phi_{1}\right)_{Q}$ has a left inverse, that is, $M_{Q}$ is a direct summand of $F_{Q}$. Then $D \otimes_{A} A_{Q}$ is smooth over $C \otimes_{A} A_{Q}$, hence smooth over $C$. Therefore $D_{P} \equiv\left(D \otimes_{A} A_{Q}\right)_{\left(P D \otimes_{A} A_{Q}\right)}$ is smooth over $C$, so $H_{D / A} \not \subset P$, as desired.

Next, we prove a variation of Proposition 3.4 which will be used in the proof of Theorem 1.2. Consider a diagram (1.1) where $C=\frac{A\left[u_{1}, \ldots, u_{n}\right]}{\left(f_{1}, \ldots, f_{r}\right)}$ is defined by linear homogeneous equations (3.6). Assume that $A$ is noetherian. Let $m$ be a prime ideal of $A$ such that $m A_{m} \subset \sqrt{\Delta_{f}^{0} A_{m}}$. The point of the following proposition is that we can resolve the singularitites lying over $m$ by a homomorphism $\phi: C \rightarrow D$, smooth away from $V(m)$, even though $C$ itself might not be smooth over $A$ away from $V(m)$.

Proposition 3.6. Let $M, K$ and $u \in K e r\left(d_{1}^{*} \otimes B\right)$ be as in Proposition 3.4. Assume that $u \in \operatorname{Im}(\alpha \otimes B)$ in (3.3). There exists a commutative diagram (1.2) such that $D$ is defined by linear homogeneous equations over $A, m D \subset H_{D / C}$ and $H_{D / A} \cap A \not \subset m$ (i.e. $D \otimes_{A} A_{m}$ is smooth over $A$ ).

Proof. Our strategy is first to factor $\tau$ as $A \rightarrow C^{\prime} \rightarrow C$, where $C^{\prime}$ is an $A$-algebra such that $m C^{\prime} \subset H_{C^{\prime} / A}$, apply Proposition 3.4 to $C^{\prime}$, and then take the base change of the resulting homomorphism $C^{\prime} \rightarrow D^{\prime}$ by $C$. Let $f$ denote the column $r$-vector with entries $f_{j}, 1 \leq j \leq r$, and $a_{i}$ the column $r$-vector with entries $a_{i j}, 1 \leq j \leq r$, so that (3.6) can be written in the form $f=\sum_{i=1}^{n} a_{i} u_{i}$.

Lemma 3.7. Let $A$ be a ring and $a_{1}, \ldots, a_{n}$ r-vectors with entries in $A$. Let $\Delta$ denote the ideal generated by all the $r \times r$ minors of the $r \times n$ matrix formed by $a_{1}, \ldots, a_{n}$ (as usual, we take $\Delta=0$ if $n<r$ ). Let $J$ denote the submodule of $A^{r}$ generated by $a_{1}, \ldots, a_{n}$. Then $\Delta A^{r} \subset J$.

Proof. For an $r \times r$ minor $\Delta_{1}$ of the matrix $\left(a_{i j}\right)_{\substack{1 \leq i \leq r \\ 1 \leq j \leq n}}$ and an integer $q, 1 \leq q \leq r$, let $b\left(\Delta_{1}, q\right)$ denote the $r$-vector whose $q$ th entry is $\Delta_{1}$ and all the other entries 0 . By linear algebra, $b\left(\Delta_{1}, q\right) \in J$ for every $\Delta_{1}$ and $q$. Since $\Delta$ is the ideal generated by all the different minors $\Delta_{1}$, we have $\Delta A^{r} \subset J$, as desired.

We come back to the proof of Proposition 3.6. Since $m A_{m} \subset \sqrt{\Delta_{f}^{0} A_{m}}$, by Lemma 3.7 there exists $N \gg 0$ such that

$$
m^{N} A_{m}^{r} \subset J A_{m}^{r}
$$


Let $y_{1}, \ldots, y_{s}$ be a set of generators of $m^{N}$. Define $r$-vectors $b_{i j}, 1 \leq i \leq s$, $1 \leq j \leq r$, as follows. The only non-zero entry in $b_{i j}$ is the $j$ th one, and that is equal to $y_{i}$. Let $v_{j k}$ be new variables and define an $A$-module $M^{\prime}$ by

$$
M^{\prime}:=\frac{\left(\bigoplus_{i=1}^{n} A u_{i}\right) \oplus\left(\bigoplus_{\substack{1 \leq j \leq s \\ 1 \leq k \leq r}} A v_{j k}\right)}{\left(\sum_{i=1}^{n} a_{i} u_{i}+\sum_{j, k} b_{j k} v_{j k}\right)} .
$$

We have a surjection $\pi: M^{\prime} \rightarrow M$ defined by sending all the $v_{j k}$ to 0 . Let $C^{\prime}:=S_{A} M^{\prime}$. The $A$-module homomorphism $\pi$ induces an $A$-algebra homomorphism $C^{\prime} \rightarrow C$ which we also denote by $\pi$. Let $\rho^{\prime}:=\rho \circ \pi$. By $(3.10),\left(y_{1}, \ldots, y_{s}\right) \subset$ $H_{C^{\prime} / A} \cap A$, hence $m=\sqrt{\left(y_{1}, \ldots, y_{s}\right)} \subset H_{C^{\prime} / A} \cap A$.

(3.10) defines a presentation $0 \rightarrow A^{r} \rightarrow A^{n+r s} \rightarrow M^{\prime} \rightarrow 0$ which maps to (3.1) in the obvious way. We may identify $A^{r}$ with $L_{1}$. Denoting $A^{n+r s}$ by $L_{0}^{\prime}$, we get a commutative diagram

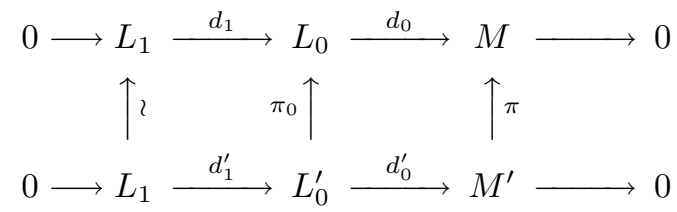

Since $\pi_{0}$ is surjective, its dual $\pi_{0}^{*}$ is injective. Hence there exists a free finitely generated $A$-module $G$ such that $\alpha: K \rightarrow K e r d_{1}^{*}$ extends to a surjection $K \oplus G \rightarrow$ $\operatorname{Ker}\left(d_{1}^{\prime}\right)^{*}$. Let $K^{\prime}:=K \oplus G$. We obtain a commutative diagram

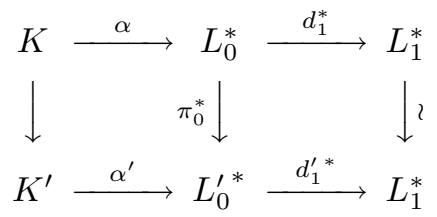

Consider the commutative diagram obtained by tensoring (3.12) with $B$. Let $u^{\prime} \in$ $\operatorname{Ker}\left(d_{1}^{\prime *} \otimes B\right)$ denote the element corresponding to $\left.\rho^{\prime}\right|_{M^{\prime}} \circ d_{1}^{\prime}$. Then $u^{\prime}=\left(\pi_{0}^{*} \otimes B\right)(u)$, hence $u^{\prime} \in\left(\pi_{0}^{*} \otimes B\right)\left((\alpha \otimes B)\left(L_{0}^{*}\right)\right) \subset\left(\alpha^{\prime} \otimes B\right)\left(K^{\prime} \otimes B\right)$. Thus $C^{\prime}$ and $\alpha^{\prime}$ satisfy the hypotheses of Proposition 3.4. Hence there exists a commutative diagram

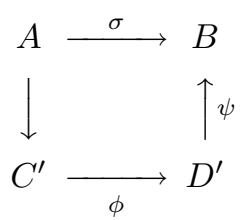

such that $D^{\prime}$ is a polynomial ring in finitely many variables over $A$ and

$$
m D^{\prime} \subset H_{C^{\prime} / A} D \subset H_{D^{\prime} / C^{\prime}}
$$

(cf. (3.7)). By construction, $D^{\prime}=S_{A} F^{\prime}$, where $F^{\prime}:=K^{\prime *}$. Define $D:=D^{\prime} \otimes_{C^{\prime}} C$. Since smoothness is preserved by base change [10, Chapter 11, (28.E), p. 201], we have

$$
H_{D^{\prime} / C^{\prime}} D \subset H_{D / C}
$$

Combining (3.13) and (3.14), we obtain $m D \subset H_{D / C}$. By (3.9), $\pi_{m}: M_{m}^{\prime} \rightarrow M_{m}$ has a right inverse; in fact, $M_{m}^{\prime} \cong M_{m} \oplus F_{1}$, where $F_{1} \cong A_{m}^{r s}$. Then $\left(\pi_{0}\right)_{m}$ has a 
right inverse, so $\left(\pi_{0}^{*}\right)_{m}$ has a left inverse. Hence, $K_{m}^{\prime} \cong K_{1} \oplus F_{1}^{*}$ where $K_{1}$ is a free finite $A_{m}$-module which surjects onto $\left(\pi_{0}^{*}\right)_{m}\left(\operatorname{Ker}\left(d_{1}^{*}\right)_{m}\right)$. Then $F_{m}^{\prime} \cong K_{1}^{*} \oplus F_{1}$. Since the map $\pi_{m}$ is just the quotient by $F_{1}, D \otimes_{A} A_{m}=\frac{D^{\prime} \otimes_{A} A_{m}}{F_{1} D^{\prime} \otimes_{A} A_{m}}=S_{A_{m}}\left(K_{1}^{*}\right)$, so $D \otimes_{A} A_{m}$ is smooth over $A_{m}$. Therefore $H_{D / A} \cap A \not \subset m$, as desired.

We end this section by proving Proposition 1.6.

Proof of Proposition 1.6. The first step is to reduce to the case when $A^{*}$ is of finite type over $A$ (we prove a slightly more general result for future use).

Lemma 3.8. Assume that $A^{*}=\lim _{\vec{\alpha}} A_{\alpha}$, where $\left\{A_{\alpha}\right\}$ is a direct system of finite type A-subalgebras, such that, for all $\alpha, A^{*}$ is flat over $A_{\alpha}$ and

$$
H_{A^{*} / A} B \subset H_{A_{\alpha} / A} B \text {. }
$$

Then there exists a finite type A-subalgebra $A_{0}^{*} \subset A^{*}$, such that all the homomorphisms in (1.7) are defined over $A_{0}^{*}$, so that (1.7) descends to a commutative diagram of finite type $A_{0}^{*}$-algebras mapping to $B$, and such that conditions (1)-(8) still hold for the descended diagram.

Proof. Let $A_{0}^{*}$ be one of the $A_{\alpha}$ such that the diagram (1.7), $\operatorname{Ker} \lambda^{*}$, the presentation of condition (5) and the presentation (1.4) of $M$ are defined already over $A_{0}^{*}$. Then condition (5) of Proposition 1.6 holds for such an $A_{0}^{*}$. It is clear that conditions (2), (4), (6) and (7) of Proposition 1.6 hold for $A_{0}^{*}$ if $A_{0}^{*}$ is sufficiently large. Condition (1) holds for $A_{0}^{*}$ by (3.15). Moreover, choose $A_{0}^{*}$ large enough so that all the presentations used in the calculation of all the Jacobian ideals in conditions (3) and (8), are defined already over $A_{0}^{*}$. Let $C_{1}^{*}$ and $C_{10}^{*}$ be the $A_{0}^{*}$-algebras such that $C^{*}=C_{1}^{*} \otimes_{A_{0}^{*}} A^{*}$ and $C_{0}^{*}=C_{10}^{*} \otimes_{A_{0}^{*}} A^{*}$. Let $C_{1}^{*}=\frac{C_{10}^{*}[V]}{J}$ be a presentation of $C_{1}^{*}$ over $C_{10}^{*}$, and $g=\left(g_{1}, \ldots, g_{r}\right)$ an $r$-tuple of elements of $J$. Since $A^{*}$ is flat over $A_{0}^{*}, C^{*}$ is flat over $C_{1}^{*}$, and hence $((g): J) C^{*}=\left((g) C^{*}\right):\left(J C^{*}\right)$. By definition of $A_{0}^{*}$, the ideal $\Delta_{g}$ is the same whether computed in $C_{10}^{*}[V]$ or in $C_{0}^{*}[V]$. Thus $H_{C^{*} / C_{0}^{*}}=H_{C_{1}^{*} / C_{10}^{*}} C^{*}$, hence condition (8) holds for $A_{0}^{*}$. Condition (3) is proved in exactly the same way. This proves Lemma 3.8 .

Now, since $A^{*}$ is essentially of finite type over $A$, we may write $A^{*}=A_{S}^{\prime}$, where $A^{\prime}$ is of finite type over $A$ and $S$ is a multiplicative subset of $A^{\prime}$. Let the direct system $\left\{A_{\alpha}\right\}$ be $\left\{A_{S^{\prime}}^{\prime}\right\}$, where $S^{\prime}$ ranges over all the finite subsets of $S$. For each $\alpha, A^{*}$ is smooth over $A_{\alpha}$; in particular, it is flat. We have $H_{A^{*} / A}=H_{A_{\alpha} / A} A^{*}=H_{A^{\prime} / A} A^{*}$, so (3.15) holds. Thus, Lemma 3.8 applies. Replacing $A^{*}$ by $A_{0}^{*}$ of Lemma 3.8 , we may assume that $A^{*}$ is of finite type over $A$. Now, apply Proposition 3.6 to the $A^{*}$-algebra $C_{0}^{*}$. We obtain a commutative diagram

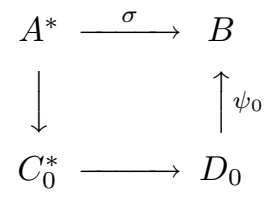

where

$$
\begin{aligned}
& H_{D_{0} / A^{*}} \cap A^{*} \not \subset m^{*} \text { and } \\
& m^{*} D_{0} \subset H_{D_{0} / C_{0}^{*}} .
\end{aligned}
$$


Put $D^{*}:=D_{0} \otimes_{C_{0}^{*}} C^{*}$ and $D:=D_{0} \otimes_{C_{0}^{*}} C_{N}^{*}$. We get a commutative diagram

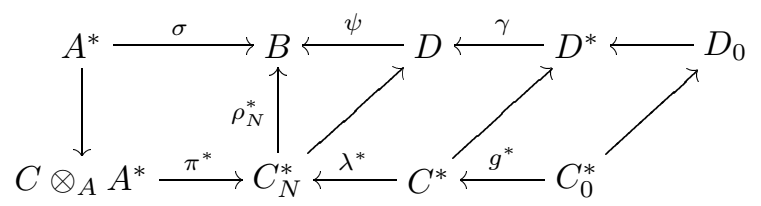

extending (1.7), where $\psi$ is the natural map coming from the tensor product; this gives a diagram (1.2). We now show that this diagram (1.2) satisfies the conclusion of Theorem 1.5 and (1.3). Since $C_{P \cap C^{*}}^{*}$ is smooth over $\left(C_{0}^{*}\right)_{P \cap C_{0}^{*}}$ by $(1.14), D_{P \cap D^{*}}^{*}$ is smooth over $\left(D_{0}^{*}\right)_{P \cap D_{0}^{*}}$ by base change, and hence also over $A^{*}$ and $A$ by (1.8), (3.17) and transitivity of smoothness [10, Chapter 11, (28.E), p. 201]. Next, we use (1.11)-(1.13) to show that the natural surjective map $\gamma_{P}: D_{P \cap D^{*}}^{*} \rightarrow D_{P \cap D}$ is actually an isomorphism.

Lemma 3.9. Let $A \rightarrow D$ be a local homomorphism of local noetherian rings with $D$ smooth over $A$. Let $m$ be an ideal of $A$. Let I be a proper ideal of $D$ such that $I \cap A=(0)$ and

$$
m D \subset \sqrt{\left(I^{2}: I\right) D} .
$$

Then $m D \subset \sqrt{A n n_{D} I}$.

Proof. Since $A \rightarrow D$ is local and $D$ is smooth over $A$, the minimal primes of $D$ are precisely the extensions to $D$ of the minimal primes of $A$. Let $P_{0}$ be a minimal prime of $A$; then $P_{0} D$ is a minimal prime of $D$. We claim that

$$
m I \subset P_{0} D .
$$

If $m \subset P_{0}$, there is nothing to prove. Assume that $m \not \subset P_{0}$; take an element $x \in m \backslash P_{0}$. The inclusion (3.20) still holds after tensoring over $A$ with $\frac{A_{x}}{P_{0} A_{x}}$; the left hand side of (3.20) becomes the unit ideal, hence so does the right hand side. We obtain

$$
I \frac{D}{P_{0} D} \otimes_{A} A_{x} \subset I^{2} \frac{D}{P_{0} D} \otimes_{A} A_{x}
$$

in the noetherian ring $\frac{D}{P_{0} D} \otimes_{A} A_{x}$ without zero divisors. Moreover we claim that

$$
I \frac{D}{P_{0} D} \otimes_{A} A_{x} \neq \frac{D}{P_{0} D} \otimes_{A} A_{x} .
$$

Indeed, equality in (3.23) would mean that $x^{T}=a y+b$, for some $T \in \mathbb{N}, a \in P_{0}$, $y \in D$ and $b \in I$. Let $z$ be an element of $A$, contained in all the minimal primes of $A$ except $P_{0}$. Then $z a$ is nilpotent. Then for $S \in \mathbb{N}$ sufficiently large, we have $\left(z x^{T}\right)^{S}=(a z y+z b)^{S} \in I \cap A \backslash P_{0}$, which is a contradiction. (3.22) and (3.23) prove that $I \frac{D}{P_{0} D} \otimes_{A} A_{x}=(0)$; by the choice of $x, I \subset P_{0} D$ and (3.21) is proved. Since this holds for every minimal prime $P_{0}$, we obtain that $m I$ is nilpotent. Say, $(m I)^{L}=(0)$. By (3.20), there is an $N \in \mathbb{N}$ such that $m^{N} I \subset I^{2}$. Iterating this $L-1$ times, we obtain $m^{N L} I \subset m^{L} I^{L}=(0)$. We have found a power of $m$ annihilating $I$, as desired.

We continue with the proof of Proposition 1.6. Extending all the ideals in (1.11)(1.12) to $D_{P \cap D^{*}}^{*}$, we obtain

$$
I D_{P \cap D^{*}}^{*} \subset\left(m^{*}\right)^{N} D_{P \cap D^{*}}^{*}
$$


and $m^{*} D_{P \cap D^{*}}^{*} \subset \sqrt{\left(I^{2}: I\right) D_{P \cap D^{*}}^{*}}$. By Lemma 3.9, applied to the smooth, local $A_{m^{*}}^{*}$-algebra $D_{P \cap D^{*}}^{*}$, we have

$$
m^{*} D_{P \cap D^{*}}^{*} \subset \sqrt{A n n_{D_{P \cap D^{*}}^{*}} I D_{P \cap D^{*}}^{*}} .
$$

In other words, $I D_{P \cap D^{*}}^{*}$ is annihilated by some power of $m^{*}$, so that

$$
I D_{P \cap D^{*}}^{*} \subset A n n_{P \cap D^{*}}\left(m^{*}\right)^{\infty} D_{P \cap D^{*}}^{*}
$$

Combining (3.24) and (3.26), we obtain

$$
\begin{array}{r}
\text { Ker } \gamma_{P} \equiv I D_{P \cap D^{*}}^{*} \subset\left(\operatorname{Ann}_{D_{P \cap D^{*}}^{*}}\left(m^{*}\right)^{\infty} D_{P \cap D^{*}}^{*}\right) \cap\left(\left(m^{*}\right)^{N} D_{P \cap D^{*}}^{*}\right) \\
=\left(\left(A n n_{A_{m^{*}}^{*}}\left(m^{*}\right)^{\infty} A_{m^{*}}^{*}\right) \cap\left(\left(m^{*}\right)^{N} A_{m^{*}}^{*}\right)\right) D_{P \cap D^{*}}^{*},
\end{array}
$$

where the last equality holds since $D_{P \cap D^{*}}^{*}$ is faithfully flat over $A_{m^{*}}^{*}$ (being smooth and local). Now, $A_{m^{*}}^{*}$ is smooth over $A_{m}$ by (1.8), hence fatithfully flat over it. Then all the associated primes of $A_{m^{*}}^{*}$ are extended from associated primes of $A_{m}$, in particular, are contained in $m A_{m^{*}}^{*}$. If $m^{*} A_{m^{*}}^{*} \not \subset m A_{m^{*}}^{*}$, then it contains some non-zero divisors, so that $A n n_{A^{*}}\left(m^{*}\right)^{\infty} A_{m^{*}}^{*}=(0)$ and so $\operatorname{Ker} \gamma_{P}=(0)$ by (3.27). Assume that $m^{*} A_{m^{*}}^{*} \subset m A_{m^{*}}^{*} ;$ the opposite inclusion is trivial by definitions, so that $m A_{m^{*}}^{*}=m^{*} A_{m^{*}}^{*}$. Hence

$$
\begin{aligned}
\left(A n n_{A_{m^{*}}^{*}}(m *)^{\infty} A_{m^{*}}^{*}\right) \cap\left((m *)^{N} A_{m^{*}}^{*}\right) & =\left(A n n_{A_{m^{*}}^{*}} m^{\infty} A_{m^{*}}^{*}\right) \cap m^{N} A_{m^{*}}^{*} \\
= & \left(\left(A n n_{A_{m}} m^{\infty} A_{m}\right) \cap m^{N} A_{m}\right) A_{m^{*}}^{*},
\end{aligned}
$$

where the last equality holds by faithful flatness of $A_{m^{*}}^{*}$ over $A_{m}$. Now, (1.13), (3.27) and (3.28) prove that $\operatorname{Ker} \gamma_{P}=(0)$, so that $\gamma_{P}$ is an isomorphism. Since $D_{P \cap D} \cong D_{P \cap D^{*}}^{*}$ is smooth over $A_{m^{*}}^{*}$ and $A_{m^{*}}^{*}$ over $A(1.8), D_{P \cap D}$ is smooth over $A$. This gives (2) of Theorem 1.5.

Moreover, from (3.18) we get $m^{*} D \subset H_{D / C_{N}^{*}}$ by base change. Combined with (1.8)-(1.10), transitivity of smoothness and the fact that $H_{A / A^{*}} C \subset H_{C \otimes_{A} A^{*} / C}$ (base change), we get

$$
\sqrt{H_{C / A} B} \subset P \subset \sqrt{H_{D / C_{N}} H_{C_{N} /\left(C \otimes_{A} A^{*}\right)} H_{A^{*} / A} B} \subset \sqrt{H_{D / C} B},
$$

which gives (1.3). (1) of Theorem 1.5 follows from (1.3) and Property 2.16.

Remark 3.10. If $B$ is local with maximal ideal $P$, then, replacing $D$ by $D_{x}$, where $x$ is any element of $H_{D / A} \backslash(P \cap D)$, we get a diagram (1.2) with $D$ smooth over $A$, proving Theorem 1.2.

\section{§4. Smoothing of An isolated, Almost Complete intersection SINGULARITY WITH NO RESIDUE FIELD EXTENSION}

In this section we prove a special case of of Theorem 1.2: Proposition 1.8. Extending the proof from this special case to the general one forms the technical part of the paper and occupies $\S 5-\S 9$. Our main tool in proving Proposition 1.8 will be a transformation of $C$ called generalized blowing up. The map $\pi^{*}$, required in the diagram (1.7), will be given as a composition of generalized blowings up. We start by defining generalized blowing up and studying its basic properties. Consider a diagram (1.1), with $C$ of finite type over $A$. Let $m$ be an ideal of $A$ and $z_{1}, \ldots, z_{k}$ a set of generators of $m$. Fix a presentation $C=\frac{A[u]}{I}$. Assume that

$$
\rho(C) \subset \sigma(A)+m B
$$


(note that (4.1) is satisfied in the situation of Proposition 1.8 by the conditions (2) and (4)). We now define the generalized blowing up $\pi: C \rightarrow C_{1}$ of $C$ along $m$. By (4.1), there exist $c_{1}, \ldots, c_{n} \in A$ such that $\rho\left(u_{i}\right)-\sigma\left(c_{i}\right) \in m B$. Let $v_{i j}, 1 \leq i \leq n$, $1 \leq j \leq k$ be independent variables and consider the change of variables

$$
u_{i}-c_{i}=\sum_{j=1}^{k} z_{j} v_{i j}, \quad 1 \leq i \leq n .
$$

Write $v$ for $\left\{v_{i j} \mid 1 \leq i \leq n, 1 \leq j \leq k\right\}$. Equations (4.2) define a homomorphism $\pi_{z}: A[u] \rightarrow A[v]$. Put

$$
C_{1}:=\frac{A[v]}{I_{1}},
$$

where $I_{1}$ is the ideal of $A[v]$ generated by the set $\left\{\pi_{z}(f) \mid f \in I\right\}$. The homomorphism $\pi_{z}$ induces a homomorphism $C \rightarrow C_{1}$ which by abuse of notation we shall also call $\pi_{z}$. By definition of $c_{i}$, there exists a homomorphism $\rho_{1}: C_{1} \rightarrow B$ compatible with $\sigma, \tau$ and $\rho$.

Definition 4.1. The homomorphism $\pi_{z}$ is called a generalized blowing up of $C$ along $m$ (with respect to $z$ ). We emphasize that the generalized blowing up $\pi_{z}$ is a transformation which, given a finite type $A$-algebra $C$ together with a fixed presentation and a set of generators $z$ of $m$, produces a finite type $A$-algebra $C_{1}$ together with the presentation (4.3).

Remark 4.2. Note that even once we fix a presentation of $C$ and the set $z$, the map $\rho_{1}: C_{1} \rightarrow B$ is, in general, not unique. In the applications we always pick and fix one such map $\rho_{1}$. Note also that we allow the possibility $m=(0)$ in Definition 4.1. In this case, we have $k=0$ and $z=\emptyset$ and the right hand side of (4.2) is 0 .

An important property of generalized blowing up, which follows immediately from (4.2), is that

$$
m \subset H_{C_{1} / C} \cap A ;
$$

this will be used to deduce (3) of Proposition 1.6. The key idea in the proof of Proposition 1.8 (and Theorems 1.2 and 1.5) is to study the behaviour of Jacobian ideals under generalized blowing up. First, we consider the effect of the change of variables (4.2) on an arbitrary column vector of elements of $A[u]$. Let $r$ be a positive integer. Let $f$ be a column $r$-vector, whose entries are elements of $A[u]$. For $1 \leq i \leq n$, let $\left(\frac{\partial f}{\partial u_{i}}\right)$ be the column $r$-vector with entries in $A[u]$, obtained from $f$ by differentiating every entry with respect to $u_{i}$. Write $f=\sum_{\alpha} a_{\alpha} u^{\alpha}$, where $\alpha$ ranges over some finite subset of $\mathbb{N}_{0}^{n}$ and $a_{\alpha} \in A^{r}$. Let $I(f)$ denote the $A$-submodule of $A^{r}$ generated by the $a_{\alpha}$. For a submodule $J$ of $A^{r}$, we will denote by $J B^{r}$ the image of $J$ in $B^{r}$ under $\sigma$, and similarly for submodules of $C^{r}$. Our main tool will be Taylor's formula:

$$
f=f(c)+\sum_{i=1}^{n} \frac{\partial f}{\partial u_{i}}(c)\left(u_{i}-c_{i}\right)+h,
$$

where $h \in(u-c)^{2} I(f) A[u]^{r}$. Let $\rho\left(\frac{\partial f}{\partial u_{i}}\right)$ denote the column $r$-vector with entries in $B$, obtained from $\left(\frac{\partial f}{\partial u_{i}}\right)$ by mapping it to $C$ by the natural map $A[u] \rightarrow C$ and 
then applying $\rho$ to every entry. Let $J(f)$ denote the submodule of $B^{r}$ generated by the $r$-vectors $\rho\left(\frac{\partial f}{\partial u_{i}}\right), 1 \leq i \leq n$. We have

$$
J(f) \subset I(f) B^{r} .
$$

Consider the generalized blowing up $\pi: C \rightarrow C_{1}$ given by (4.2). The key point is to compare $I(f)$ with $I(\pi(f))$ and $J(f)$ with $J(\pi(f))$.

Lemma 4.3. Assume that the entries of the r-vector $f$ belong to I. Then:

$$
\begin{aligned}
J(\pi(f)) & =m J(f), \\
I(\pi(f)) B^{r} & \subset m J(f)+m^{2} I(f) B^{r} .
\end{aligned}
$$

Proof. (4.7) follows from (4.2) by the chain rule. To prove (4.8), substitute (4.2) in (4.5). We obtain

$$
\pi(f)=f(c)+\sum_{i, j} \frac{\partial f}{\partial u_{i}}(c) z_{j} v_{i j}+h_{1},
$$

where $h_{1} \in m^{2}(v)^{2} I(f) A[v]^{r}$. Let $J_{0}(f)$ denote the submodule of $A^{r}$ generated by $\frac{\partial f}{\partial u_{1}}(c), \ldots, \frac{\partial f}{\partial u_{n}}(c)$. Since for each $i$ we have $\frac{\partial f}{\partial u_{i}}-\frac{\partial f}{\partial u_{i}}(c) \in(u-c) I(f) A[u]^{r}$, we have

$$
J_{0}(f) B^{r} \subset J(f)+m I(f) B^{r} .
$$

Applying $\rho$ to (4.9), identifying $f(c)$ with its image in $B$ and using that $f$ has entries in $I$ (and hence maps to 0 in $B^{r}$ ), we obtain

$$
f(c) \in\left(m J_{0}(f)+m^{2} I(f)\right) B^{r} .
$$

By (4.9)-(4.11), $I(\pi(f)) B^{r} \subset\left(m J_{0}(f)+m^{2} I(f)\right) B^{r}=m J(f)+m^{2} I(f) B^{r}$. Lemma 4.3 is proved.

Now consider a sequence

$$
C \stackrel{\pi_{1}}{\longrightarrow} C_{1} \stackrel{\pi_{2}}{\longrightarrow} \ldots \stackrel{\pi_{N}}{\longrightarrow} C_{N}
$$

of $N$ generalized blowings up along $m$. For each $i$, we have a homomorphism $\rho_{i}: C_{i} \rightarrow B$; the $\rho_{i}$ commute with the $\pi_{i}$ in (4.12). Here we are assuming that

$$
\rho_{i}\left(C_{i}\right) \subset \sigma(A)+m B
$$

for each $i<N$. Note that this assumption holds for any algebra $C_{i}$ if $m B=P$ and (2) of Proposition 1.8 is satisfied. In the next lemma, assume that $C=\frac{A[u]}{I}$ is an almost complete intersection at $P \cap C$. Let $f$ be the column $r$-vector with entries $f_{i}$, and let $J(f)$ be as above. By (1.17) and Lemma 3.7, we have

$$
m^{N} B^{r} \subset J(f)
$$

for all $N$ sufficiently large. Since $A$ is assumed to be noetherian and since the $A n n_{A} m^{i}$ form an ascending chain of ideals, $A n n_{A} m^{\infty}=A n n_{A} m^{i}$ for some $i$. Using the Artin-Rees lemma, we obtain, for all $N$ sufficiently large,

$$
m^{N} \cap\left(A n n_{A} m^{\infty}\right)=m^{N} \cap\left(A n n_{A} m^{i}\right) \subset m^{i} A n n_{A} m^{i}=(0) .
$$


Lemma 4.4. Consider a diagram (1.1). Assume that $A$ is local with maximal ideal $m$, that (1) and (3) of Proposition 1.8 hold, that $m B=P$ and that (4.13) holds for all the A-algebras $C_{i}$ appearing in (4.12), so that the sequence (4.12) is well defined (note that the last condition holds automatically if we have (2) of Proposition 1.8). Take $N \in \mathbb{N}$ in (4.12) to be sufficiently large so that both (4.14) and (4.15) hold (again, if $r=0$, we regard (4.14) as being vacuously true). Let $\pi^{*}:=\pi_{N} \circ \cdots \circ \pi_{1}$. Then the map $\pi^{*}: C \rightarrow C_{N}$ fits in a commutative diagram (1.7) (with $A=A^{*}$ and $\left.m=m^{*}\right)$, satisfying (1)-(5) and (8) of Proposition 1.6. If, in addition,

$$
m^{2 N} \subset I\left(f^{(N)}\right)
$$

then (7) of Proposition 1.6 holds.

Proof. First, we construct the diagram (1.7). By definition of generalized blowing up, each $C_{i}$ comes together with a specific presentation (cf. (4.3)); let $C_{N}=$ $\frac{A\left[u^{(N)}\right]}{I_{N}}$ be the given presentation of $C_{N}$. Let $f_{i}^{(N)}:=\left(\pi_{N} \circ \cdots \circ \pi_{2} \circ \pi_{1}\right)\left(f_{i}\right)$ for $1 \leq i \leq r$. By Lemma 4.3, (4.14) and induction on $N$, we have $I\left(f^{(N)}\right) B^{r} \subset$ $m^{N} J(f)+m^{2 N} I(f) B^{r}=m^{N} J(f)=J\left(f^{(N)}\right)$, so

$$
I\left(f^{(N)}\right) B^{r}=J\left(f^{(N)}\right)
$$

by (4.6). Let $\left(f^{(N)}\right)$ denote the ideal of $A\left[u^{(N)}\right]$, generated by $f_{1}^{(N)}, \ldots, f_{r}^{(N)}$. Put $C^{*}:=\frac{A\left[u^{(N)}\right]}{\left(f^{(N)}\right)}$. We have natural homomorphisms $\lambda^{*}: C^{*} \rightarrow C_{N}$ and $\rho^{*}: C^{*} \rightarrow B$, given by $\rho^{*}=\rho_{N} \circ \lambda^{*}$; note that $\operatorname{Ker} \lambda^{*}=I_{N} C^{*}$. Let $\left(a_{1}, \ldots, a_{l}\right)$ denote a minimal set of generators of $I\left(f^{(N)}\right)$. Write $f^{(N)}=\sum_{i=1}^{l} a_{i} g_{i}$, where $g_{i} \in A\left[u^{(N)}\right]$. Let $G_{1}, \ldots, G_{l}$ be new variables. Let $F_{1}, \ldots, F_{r}$ denote the entries of the $r$-vector $\sum_{i=1}^{l} a_{i} G_{i}$. We will write $(F)$ for $\left(F_{1}, \ldots, F_{r}\right)$. Let $C_{0}^{*}:=\frac{A\left[G_{1}, \ldots, G_{l}\right]}{(F)}$. Let $g: C_{0}^{*} \rightarrow C^{*}$ be the map which sends $G_{i}$ to $g_{i}$. This completes the construction of the diagram (1.7). Again, note that we allow the possibility $r=l=0,\left(f^{(N)}\right)=(F)=$ (0). Next, we show that the diagram (1.7) thus constructed satisfies (1)-(5) and (8) of Proposition 1.6. (1) and (2) of Proposition 1.6 are trivial. (1.10) follows immediately from (4.4) and Property 2.16 by induction on $N$. From (1.18) we obtain $m C_{P \cap C^{*}}^{*} \subset \sqrt{\left(\left(I_{N}^{2}\right): I_{N}\right) C_{P \cap C^{*}}^{*}}$; this gives (1.12). (1.13) is nothing but (4.15). (5) of Proposition 1.6 is true by definition. It remains to prove (8).

Proof of (8). Let $K=\frac{B}{P}$. Let $\frac{\partial g}{\partial u^{(N)}}$ denote the $l \times n$ matrix whose $i j$ th entry is $\frac{\partial g_{i}}{\partial u_{j}^{(N)}}$. Since $J\left(f^{(N)}\right)$ is generated by $\rho_{N}\left(\frac{\partial f^{(N)}}{\partial u_{i}^{(N)}}\right)=\sum_{j=1}^{l} a_{j} \rho_{N}\left(\frac{\partial g_{j}}{\partial u_{i}^{(N)}}\right), 1 \leq i \leq n$, and since, by (4.17) and Nakayama's lemma, $\left(a_{1}, \ldots, a_{l}\right)$ induces a minimal set of generators of the $K$-vector space $\frac{J\left(f^{(N)}\right)}{P J\left(f^{(N)}\right)}$, we have

$$
\operatorname{rk}\left(\frac{\partial g}{\partial u^{(N)}}\right)=l \quad \bmod \left(P \cap A\left[u^{(N)}\right]\right) .
$$

Since $C^{*}$ is defined over $C_{0}^{*}$ by the equations $g_{i}=G_{i}, 1 \leq i \leq l,(4.18)$ implies that $C_{P \cap C^{*}}^{*}$ is smooth over $C_{0}^{*}$, that is, $H_{C^{*} / C_{0}^{*}} \not \subset P \cap C^{*}$, as desired. Again, the above is trivially true if $r=0$, for then $C^{*}=C_{0}^{*}=A\left[u^{(N)}\right]$. 
Finally, suppose (4.16) holds. By definitions, $\Delta_{F}^{0}$ is the ideal generated by all the $r \times r$ minors of the $r \times l$ matrix formed by $a_{1}, \ldots, a_{l}$, so (4.16) implies that $m \subset \sqrt{\Delta_{F}^{0}}$, as desired. This completes the proof of Lemma 4.4 .

Proof of Proposition 1.8. Put $A^{*}=A_{m}$. From now on, to simplify the notation, we will replace $A$ by $A^{*}, C$ by $C \otimes_{A} A^{*}$, and assume that $A$ is local with maximal ideal $m$ (in particular, $\sigma$ is faithfully flat). Under this assumption, we will construct a diagram (1.7) with $A=A^{*}$. Take $N$ as in Lemma 4.4 and consider the sequence (4.12) of $N$ generalized blowings up. By Lemma 4.4, (1)-(5) and (8) of Proposition 1.6 are satisfied. Proposition 1.6 (6) holds by Remark 3.1, since $B$ is flat over $A$. It remains to prove (4.16) to infer (7).

Lemma 4.5. If $B$ is flat over $A$, then (4.16) holds.

Proof. We have $m^{2 N} B^{r} \subset J\left(f^{(N)}\right) B^{r}$ by (4.7), (4.14), and induction on $N$. By (4.17), this gives $m^{2 N} B^{r} \subset I\left(f^{(N)}\right) B^{r}$. (4.16) follows by faithful flatness of $B$ over $A$. This completes the proof of Proposition 1.8.

Remark 4.6. Suppose that assumption (4) in Proposition 1.8 is replaced by saying that $\sigma: A \rightarrow B$ is formally smooth in the $P$-adic topology (by Proposition 2.7, this is weaker than being a regular homomorphism). Then the conclusion of Proposition 1.8 still holds: we have only to reduce to the situation when $m B=P$. This can be done as follows. Let $K:=\frac{B}{P} \cong \frac{A_{m}}{m}$ and $B_{0}:=\frac{B}{m B}$. The map $K \rightarrow B_{0}$ induced by $\sigma$ is formally smooth in the $P B_{0}$-adic topology (by base change: [10, Chapter 11, (28.E), p. 201]), so $B_{0}$ is a regular local ring (Proposition 2.6). Let $x_{1}, \ldots, x_{d}$ be elements of $B$ which induce a regular system of parameters of $B_{0}$ and let $X_{1}, \ldots, X_{d}$ be independent variables. Write $X$ for $X_{1}, \ldots, X_{d}$. Consider the map $\sigma_{X}: A[X]_{P \cap X} \rightarrow B$ which sends $X_{i}$ to $x_{i}$. The map $\sigma_{X}$ is flat by Corollary 2.22. Now we can apply Proposition 1.8 to $\sigma_{X}$. This gives diagram (1.7) with $A^{*}=A[X]_{P \cap A[X]}$. Since $A[X]_{m}$ is smooth over $A$, (1.8) is satisfied and we are done.

\section{$\S 5$. Smoothing of An ISOlated Singularity over A LOCAL RING WITH NO RESIDUE FIELD EXTENSION}

Consider a diagram (1.1). Let $P$ be a minimal prime of $H_{C / A} B$ and let $m:=$ $P \cap A$. In this section we prove

Proposition 5.1. Assume that (1), (2) and (4) of Proposition 1.8 hold. Then there exists a diagram (1.7) satisfying conditions (1)-(8) of Proposition 1.6 (and hence the conclusion of Theorem 1.2 holds in this case).

With a view to $\S \S 8-9$, we will start out working under more general hypotheses than those of Proposition 5.1, and gradually impose more restrictions on our diagram (1.1) as we go along. The idea is to show that $\rho$ factors through a map $C \rightarrow C_{L}$ such that $P \subset \sqrt{H_{C_{L} / C} B}$ and $C_{L}$ is an almost complete intersection over $A$ at $P \cap C_{L}$. Once this is done, we will invoke Proposition 1.8 and the proof will be complete. We start with any diagram (1.1) whatsoever of noetherian rings. Let $C=\frac{A\left[u_{1}, \ldots, u_{n}\right]}{I}$ be a presentation of $C$. Let $\frac{\mathcal{I}}{\mathcal{I}^{2}}$ denote the coherent sheaf on Spec $C$ such that $\Gamma\left(\operatorname{Spec} C, \frac{\mathcal{I}}{\mathcal{I}^{2}}\right)=\frac{I}{I^{2}}$ (in what follows we will adopt the following convention: ideals and modules will be denoted by capital letters, and their sheafications by script capital letters). Restricted to the smooth locus of $C$ over $A$, the 
sheaf $\left.\frac{\mathcal{I}}{\mathcal{I}^{2}}\right|_{\text {Spec } C \backslash V\left(H_{C / A}\right)}$ is nothing but the conormal bundle of Spec $C \backslash V\left(H_{C / A}\right)$ in Spec $A[u]$. The first step of the proof is to achieve the situation when the vector bundle $\left.\frac{\mathcal{I}}{\mathcal{I}^{2}}\right|_{\operatorname{Spec} C \backslash V\left(H_{C / A}\right)}$ is trivial. This is given by Elkik's lemma, which we now invoke. Let $C^{\prime}:=S_{C}\left(\frac{I}{I^{2}}\right)$. Extend $\rho$ to $C^{\prime}$ by setting it to be (for example) the 0 map on the positive degree part of $S_{C}\left(\frac{I}{I^{2}}\right)$.

Lemma 5.2 (Elkik [7, Lemma 3]). There exists a presentation $C^{\prime}=\frac{A\left[u^{\prime}\right]}{I^{\prime}}$ such that the restriction $\left.\frac{\mathcal{I}^{\prime}}{\mathcal{I}^{\prime 2}}\right|_{\operatorname{Spec} C^{\prime} \backslash V\left(H_{C^{\prime} / A}\right)}$ is the trivial vector bundle.

Since $\left.\frac{\mathcal{I}}{\mathcal{I}^{2}}\right|_{\operatorname{Spec} C \backslash V\left(H_{C / A}\right)}$ is locally free, for any prime ideal $Q \subset C^{\prime}, C_{Q}^{\prime}$ is smooth over $C$ whenever $C_{Q \cap C}$ is smooth over $A$. Therefore

$$
H_{C / A} C^{\prime} \subset H_{C^{\prime} / C}
$$

(this will be needed to prove (1.10)). Let $P$ be as in Theorem 1.5 and let $m=$ $P \cap A$. If $H_{C^{\prime} / A} B \not \subset P$, we may take $C_{L}=D=C^{\prime}$ and there is nothing more to prove. If $H_{C^{\prime} / A} B \subset P$, then $P$ is a minimal prime of $H_{C^{\prime} / A} B$ by Property 2.16 and (5.1). In this case, replace $C$ by $C^{\prime}$. From now on, assume that the vector bundle $\left.\frac{\mathcal{I}}{\mathcal{I}^{2}}\right|_{\operatorname{Spec} C \backslash V\left(H_{C / A}\right)}$ is trivial. If $V\left(m H_{C / A} C_{P \cap C}\right) \neq \operatorname{Spec} C_{P \cap C}$, let $r:=\left.\operatorname{rk} \frac{\mathcal{I}}{\mathcal{I}^{2}}\right|_{\operatorname{Spec} C \backslash V\left(H_{C / A}\right)}$ and let $\bar{f}_{1}, \ldots, \bar{f}_{r}$ be global sections of $\left.\frac{\mathcal{I}}{\mathcal{I}^{2}}\right|_{\operatorname{Spec} C \backslash V\left(H_{C / A}\right)}$, which generate $\left.\frac{\mathcal{I}}{\mathcal{I}^{2}}\right|_{\text {Spec } C \backslash V\left(H_{C / A}\right)}$. If $V\left(m H_{C / A} C_{P \cap C}\right)=$ Spec $C_{P \cap C}$, set $r=0$ and let $\left\{\bar{f}_{1}, \ldots, \bar{f}_{r}\right\}$ be the empty set. Let $z_{1}, \ldots, z_{k}$ be a set of generators of $m$. To achieve the situation when $C$ is an almost complete intersection at $P \cap C$, we iterate the generalized blowing up $\pi_{z}$ (see Definition 4.1). Consider a sequence

$$
C \stackrel{\pi_{1}}{\longrightarrow} C_{1} \stackrel{\pi_{2}}{\longrightarrow} \ldots \stackrel{\pi_{L}}{\longrightarrow} C_{L} \stackrel{\pi_{L+1}}{\longrightarrow} \cdots
$$

of generalized blowings up (we are assuming that (4.13) holds for each $i$ in (5.2), so that (5.2) is well defined). Let $C_{i}=\frac{A\left[u_{1}^{(i)}, \ldots, u_{n_{i}}^{(i)}\right]}{I_{i}}$ be the presentation of $C_{i}$ obtained, recursively, from the definition of generalized blowing-up (here $n_{i+1}=k n_{i}$; cf. (4.2) and (4.3)). The purpose of the next several lemmas is to show that, under some additional hypotheses, after a finite number $L$ of such blowings up we can ensure that there exist $f_{1}, \ldots, f_{r} \in I_{L}$ such that for $1 \leq i \leq r, \bar{f}_{i}$ is the natural image of $f_{i}$, up to multiplication by an element of $A\left[u^{(L)}\right] \backslash\left(P \cap A\left[u^{(L)}\right]\right)$. This will mean that $C_{L}$ is an almost complete intersection over $A$ at $P \cap C$ and our proof will be finished.

Lemma 5.3. For all $i \in \mathbb{N}$, the vector bundle $\left.\frac{\mathcal{I}_{i}}{\mathcal{I}_{i}^{2}}\right|_{\operatorname{Spec} C_{i} \backslash V\left(m H_{C / A} C_{i}\right)}$ is trivial.

Proof. By definition,

$$
\frac{I_{i}}{I_{i}^{2}} \cong \frac{I_{i-1} A\left[u^{(i)}\right]}{I_{i-1}^{2} A\left[u^{(i)}\right]}, \quad i \in \mathbb{N} .
$$

Since $A\left[u^{(i)}\right]$ is flat over $A\left[u^{(i-1)}\right]$ away from $V\left(m A\left[u^{(i-1)}\right]\right)$, away from that locus (5.3) can be rewritten as

$$
\left.\left.\frac{\mathcal{I}_{i}}{\mathcal{I}_{i}^{2}}\right|_{\operatorname{Spec} C_{i} \backslash V\left(m C_{i}\right)} \cong \pi_{i}^{*}\left(\frac{\mathcal{I}_{i-1}}{\mathcal{I}_{i-1}^{2}}\right)\right|_{\operatorname{Spec~} C_{i} \backslash V\left(m C_{i}\right)}, \quad i \in \mathbb{N},
$$

and the lemma follows immediately by induction on $i$. 
For each $i$, consider the exact sequence $\frac{I_{i}}{I_{i}^{2}} \stackrel{d_{i}}{\longrightarrow} C_{i}^{n_{i}} \stackrel{\omega_{i}}{\longrightarrow} \Omega_{C_{i} / A}$ of $C_{i}$-modules and the corresponding exact sequence

$$
\frac{\mathcal{I}_{i}}{\mathcal{I}_{i}^{2}} \stackrel{\tilde{d}_{i}}{\longrightarrow} \mathcal{O}_{\text {Spec } C_{i}}^{n_{i}} \stackrel{\tilde{\omega}_{i}}{\longrightarrow} \Omega_{\text {Spec } C_{i} / A}^{1}
$$

of sheaves of $\mathcal{O}_{\text {Spec } C_{i}}$-modules. First, let $i=0$ in (5.5). Since $\bar{f}_{1}, \ldots, \bar{f}_{r} \in$ $\Gamma\left(\operatorname{Spec} C \backslash V\left(H_{C / A}\right), \frac{\mathcal{I}}{\mathcal{I}^{2}}\right)$, we have $\tilde{d}_{0}\left(\bar{f}_{j}\right) \in \Gamma\left(\operatorname{Spec} C \backslash V\left(H_{C / A}\right), \mathcal{O}_{\text {Spec } C}^{n}\right), 1 \leq$ $j \leq r$, and hence

$$
H_{C / A}^{L_{1}} \tilde{d}_{0}\left(\bar{f}_{j}\right) \in \Gamma\left(\operatorname{Spec} C, \mathcal{O}_{\text {Spec } C}^{n}\right) \equiv C^{n}, 1 \leq j \leq r \text {, for all } L_{1} \gg 0 .
$$

Lemma 5.4. Assume there is a positive integer $L_{0}$ such that

$$
m^{L_{0}}\left(C_{L_{0}}\right)_{P \cap C_{L_{0}}} \subset m H_{C / A}\left(C_{L_{0}}\right)_{P \cap C_{L_{0}}} .
$$

Let $L_{1}$ be such that (5.6) holds and let $L=L_{0} L_{1}$. Then $C_{L}$ is an almost complete intersection over $A$ at $P \cap C_{L}$.

Proof. We have

$$
m\left(C_{L}\right)_{P \cap C_{L}} \subset\left(H_{C_{L} / A}\right)_{P \cap C_{L}}
$$

by Property 2.16, induction on $L$ and (5.7). Combining Lemma 5.3, (5.4) and (5.7), we see that the sections

$\left(\pi_{L} \circ \cdots \circ \pi_{1}\right)^{*} \bar{f}_{1}, \ldots,\left(\pi_{L} \circ \cdots \circ \pi_{1}\right)^{*} \bar{f}_{r} \in \Gamma\left(\operatorname{Spec}\left(C_{L}\right)_{P \cap C_{L}} \backslash V\left(m\left(C_{L}\right)_{P \cap C_{L}}\right), \frac{\mathcal{I}_{L}}{\mathcal{I}_{L}^{2}}\right)$

freely generate $\left.\frac{\mathcal{I}_{L}}{\mathcal{I}_{L}^{L}}\right|_{\operatorname{Spec}\left(C_{L}\right)_{P \cap C_{L}} \backslash V\left(m\left(C_{L}\right)_{P \cap C_{L}}\right)}$. It remains to show (3) of Definition 1.7. This is given by the following lemma.

Lemma 5.5. There exist elements $f_{1}^{(L)}, \ldots, f_{r}^{(L)} \in I_{L}$ whose natural images in $\Gamma\left(\operatorname{Spec}\left(C_{L}\right)_{P \cap C_{L}} \backslash V\left(m\left(C_{L}\right)_{P \cap C_{L}}\right), \frac{\mathcal{I}_{L}}{\mathcal{I}_{L}^{2}}\right)$ are $\left(\pi_{L} \circ \cdots \circ \pi_{1}\right)^{*} \bar{f}_{1}, \ldots,\left(\pi_{L} \circ \cdots \circ \pi_{1}\right)^{*} \bar{f}_{r}$, respectively, up to multiplication by an element of $C_{L} \backslash P \cap C_{L}$.

Proof. Consider the commutative diagram

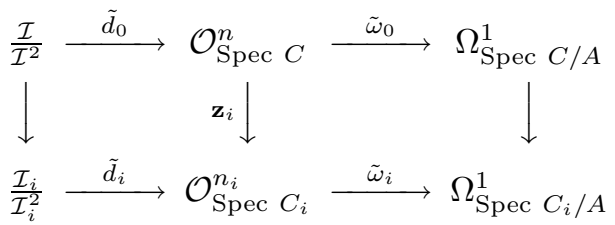

induced by the map $A[u] \rightarrow A\left[u^{(i)}\right]$, given by iterating (4.2). First, let $i=1$ in (5.9). By (4.2) and the chain rule, $\mathbf{z}_{1}$ sends an $n$-vector with entries $b_{1}, \ldots, b_{n} \in$ $\Gamma\left(U, \mathcal{O}_{\text {Spec } C}^{n}\right)$ (where $U$ is an open set of Spec $C$ ) to an $(n k)$-vector with entries $z_{j} b_{l}, 1 \leq j \leq k, 1 \leq l \leq n$. Next, let $i=L$ in (5.9). By induction on $L, \mathbf{z}_{L}$ sends $\left(b_{1}, \ldots, b_{n}\right)$ to an $n_{L}$-vector all of whose components are of the form $z^{\alpha} b_{l}$, $1 \leq l \leq n$, where $|\alpha|=L$. Combining this with (5.6)-(5.7) we obtain, after localization at $P \cap C_{L}$, that $\tilde{d}_{L}\left(\left(\pi_{L} \circ \cdots \circ \pi_{1}\right)^{*} \bar{f}_{j}\right)$ extends to an element of

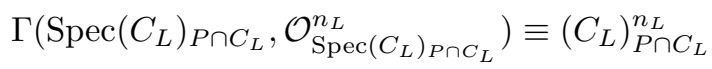

for $1 \leq j \leq r$. Since $\tilde{d}_{L}\left(\left(\pi_{L} \circ \cdots \circ \pi_{1}\right)^{*} \bar{f}_{j}\right) \in \operatorname{Ker} \tilde{\omega}_{L}$, by (5.9) there exist $\bar{f}_{1}^{(L)}, \ldots, \bar{f}_{r}^{(L)} \in \frac{I_{L}}{I_{L}^{2}} \otimes_{C_{L}}\left(C_{L}\right)_{P \cap C_{L}}$ such that $d_{L}\left(\bar{f}_{j}^{(L)}\right)=\tilde{d}_{L}\left(\left(\pi_{L} \circ \cdots \circ \pi_{1}\right)^{*} \bar{f}_{j}\right)$, 
$1 \leq j \leq r$, under the identification (5.10). Since $\tilde{d}_{L}$ is injective away from the nonsmooth locus $V\left(H_{C_{L} / A}\left(C_{L}\right)_{P \cap C_{L}}\right)$ of $\left(C_{L}\right)_{P \cap C_{L}}$, by (5.8) it is injective away from $V\left(m\left(C_{L}\right)_{P \cap C_{L}}\right)$. Since the $\left(\pi_{L} \circ \cdots \circ \pi_{1}\right)^{*} \bar{f}_{j}$ generate $\left.\frac{\mathcal{I}_{L}}{\mathcal{I}_{L}^{2}}\right|_{\operatorname{Spec}\left(C_{L}\right)_{P \cap C_{L}} \backslash V\left(m\left(C_{L}\right)_{P \cap C_{L}}\right.}$, the elements $\bar{f}_{j}^{(L)}$ generate $\frac{\mathcal{I}_{L}}{\mathcal{I}_{L}^{2}} \otimes_{C_{L}}\left(C_{L}\right)_{P \cap C_{L}}$ away from $V\left(m\left(C_{L}\right)_{P \cap C_{L}}\right)$. Since Spec $A\left[u^{(L)}\right]_{P \cap A\left[u^{(L)}\right]}$ is affine, $H^{1}\left(\operatorname{Spec} A\left[u^{(L)}\right]_{P \cap A\left[u^{(L)}\right]}, \mathcal{I}_{L}^{2}\right)=0$, so $\bar{f}_{1}^{(L)}, \ldots$, $\bar{f}_{r}^{(L)}$ can be lifted to $f_{1}^{(L)}, \ldots, f_{r}^{(L)} \in\left(I_{L}\right)_{P \cap A\left[u^{(L)}\right]} \equiv \Gamma\left(\operatorname{Spec} A\left[u^{(L)}\right]_{P \cap A\left[u^{(L)}\right]}, \mathcal{I}_{L}\right)$. Multiplying $f_{1}^{(L)}, \ldots, f_{r}^{(L)}$ by an element of $A\left[u^{(L)}\right] \backslash P \cap A\left[u^{(L)}\right]$, we may take $f_{1}^{(L)}, \ldots, f_{r}^{(L)} \in I_{L}$. This proves Lemma 5.5 and hence also Lemma 5.4.

Proof of Proposition 5.1. We want to apply Lemma 5.4. For that, we must show that there exists $L_{0}$ such that (5.7) holds. Since $P=\sqrt{H_{C / A} B}$, there exists $L_{0}$ such that

$$
m^{L_{0}} B \equiv P^{L_{0}} B \subset H_{C / A} P B \equiv m H_{C / A} B .
$$

We claim that (5.7) holds for this $L_{0}$. Indeed, let $h_{1}, \ldots, h_{t}$ be a set of generators of $H_{C / A}$. Let $h_{j}^{(i)}$ denote the image of $h_{j}$ in $C_{i}$. By (4.2), (4.5) and induction on $i$, $h_{j}^{(L)}$ can be written as

$$
h_{j}^{(L)}=g_{j}^{(L)}+q_{j}^{(L)},
$$

where $g_{j}^{(L)} \in A$ and $q_{j}^{(L)} \in m^{L} C_{L}$. Let $H_{L}^{\prime}:=\left(g_{1}^{(L)}, \ldots, g_{t}^{(L)}\right) A$.

Lemma 5.6. Let $Q$ be a module over a noetherian ring $C$ and $M$ an ideal of $C$ with $M \subset J a c(C)$. Let $H$ and $H^{\prime}$ be submodules of $Q$, with sets of generators $H=\left(h_{1}, \ldots, h_{t}\right)$ and $H^{\prime}=\left(g_{1}, \ldots, g_{t}\right)$. Assume that $g_{j}-h_{j} \in M^{L} Q, 1 \leq j \leq t$, and $M^{L} Q \subset M H^{\prime}$. Then $H=H^{\prime}$.

Proof. Since $h_{j} \in H^{\prime}+M^{L} Q, 1 \leq j \leq t$, we have $H \subset\left(H^{\prime}+M^{L} Q\right)=H^{\prime}$ and $H^{\prime} \subset H+M^{L} Q \subset H+M H^{\prime}$. The result follows by Nakayama's lemma.

By (5.11)-(5.12) and Lemma 5.6, $H_{C / A} B=H_{L_{0}}^{\prime} B$. Then (5.11) implies that $m^{L_{0}} B \subset m H_{L_{0}}^{\prime} B$, hence $m^{L_{0}} \subset m H_{L_{0}}^{\prime}$ by faithful flatness of $\sigma$, hence $m^{L_{0}} C_{L_{0}} \subset$ $m H_{L_{0}}^{\prime} C_{L_{0}}$. (5.7) follows from (5.12) and Lemma 5.6. Thus we may apply Lemma 5.4. By Lemma 5.4, $C_{L}$ is an almost complete intersection over $A$ at $P \cap C_{L}$. Apply Proposition 1.8 with $C$ replaced by $C_{L}$. We construct a diagram (1.7) (with $\left.A^{*}=A_{m}\right)$. (1.10) follows from (5.1), (4.4) and Property 2.16. (1)-(2) and (4)-(8) of Proposition 1.6 follow immediately by Proposition 1.8 and we are done.

\section{§6. Separability in Field eXtensions}

Let $\sigma:(A, m, k) \rightarrow(B, P, K)$ be a regular homomorphism of local noetherian rings. One of the difficulties in proving Theorem 1.2 comes from the fact that the residue field extension $k \rightarrow K$ induced by $\sigma$ need not be separable. However, as we shall see in $\S 7$, we always have $\operatorname{dim}_{K} H_{1}(k, K, K) \leq \operatorname{dim} B-\operatorname{dim} A<\infty$. In this section, preliminary to $\S 7$, we study field extensions with $\operatorname{dim} H_{1}(k, K, K)<\infty$. 
Notation. If $A \rightarrow B$ is a ring extension with char $A=p>0, A B^{p}$ will denote the $A$-subalgebra of $B$, generated by the set $B^{p}$. Of course, if $A$ and $B$ are fields, then $A B^{p}$ is a subfield of $B$ since, in that case, if $a \in A B^{p} \backslash\{0\}$, then $a^{-1}=$ $\left(a^{-1}\right)^{p} a^{p-1} \in A B^{p}$.

We start with a general observation about Kähler differentials in field extensions of positive characteristic. Let $k \rightarrow K$ be a field extension.

Proposition 6.1. Assume that char $k=p>0$. Let $a \in K$; consider $d a \in \Omega_{K / k}$. We have $d a=0 \Longleftrightarrow a \in k K^{p}$.

Proof. $\Longleftarrow$ is immediate.

$\Longrightarrow$ It is sufficient to consider the case when $K$ is finitely generated over $k$. From the Jacobi-Zariski sequence for the triple $k \rightarrow k K^{p} \rightarrow K$ and from the fact that $\Omega_{k K^{p} / k} \otimes_{k K^{p}} K \rightarrow \Omega_{K / k}$ is the zero map, we get the isomorphism $\Omega_{K / k K^{p}} \sim \Omega_{K / k}$. Thus, replacing $k$ by $k K^{p}$ does not change the problem, that is, we may assume, in addition, that $K^{p} \subset k$. Then $K$ can be written as $K=\frac{k\left[x_{1}, \ldots, x_{n}\right]}{\left(x_{1}^{p}-a_{1}, \ldots, x_{n}^{p}-a_{n}\right)}, a_{i} \in k$. Moreover, if $a \notin k$, then we may choose $x_{1}=a$ and therefore $d a \neq 0$.

Corollary 6.2. We have $\Omega_{K / k}=0$ if and only if $K=k K^{p}$. More generally, consider a subset $u_{\Psi}=\left\{u_{\psi} \mid \psi \in \Psi\right\} \subset K$. The elements $d u_{\psi}, \psi \in \Psi$ form a $K$-basis of $\Omega_{K / k}$ if and only if $u_{\Psi}$ is a minimal set of generators of $K$ over $k K^{p}$.

A set $u_{\Psi}$ satisfying the equivalent conditions of Corollary 6.2 is called a $p$-basis of $K$.

Remark 6.3. Let $\delta: k \rightarrow K$ be a finitely generated extension of fields of characteristic $p>0$. A $p$-basis of $K$ over $k$ can be constructed as follows. Decompose $\delta$ as $k \rightarrow K_{t} \rightarrow K_{s} \rightarrow K$, where $K_{t}$ is purely transcendental over $k, K_{s}$ is separable algebraic over $K_{t}$ and $K$ is algebraic and purely inseparable over $K_{s}$. Moreover, choose this decomposition in such a way as to minimize $\operatorname{dim}_{K} \Omega_{K / K_{t}}$. Let $u_{\Lambda}$ be a minimal set of generators of $K_{t}$ over $k$ and $v_{\Phi}$ a $p$-basis of $K$ over $K_{s}$. Then it is easy to see that $u_{\Lambda} \cup v_{\Phi}$ forms a $p$-basis of $K$ over $k$ (indeed, $d u_{\Lambda} \cup d v_{\Phi}$ generate $\Omega_{K / k}$ by definition; moreover, they are linearly independent: a non-trivial $K$-linear dependence relation among $d u_{\Lambda} \cup d v_{\Phi}$ would imply that one of the $w_{\lambda}$, $\lambda \in \Lambda$ can be removed and replaced by one of the $v_{\phi}$, which contradicts the minimality assumption on $\operatorname{dim}_{K} \Omega_{K / K_{t}}$ ). In particular, $\Omega_{K / k}=0$ if and only if $K$ is separable algebraic over $k$. If $K$ is not finitely generated over $k$, then the extension $k \hookrightarrow k\left(t^{p^{-\infty}}\right) \equiv k\left(t, t^{\frac{1}{p}}, t^{\frac{1}{p^{2}}}, \ldots\right)$, where $t$ is transcendental over $k$, provides a counterexample to all these statements.

Let $\delta: k \rightarrow K$ be any field extension (no assumptions on the characteristic). Let $\left\{w_{\lambda}\right\}_{\lambda \in \Lambda}$ be a maximal family of elements of $K$ such that

(1) $\left\{w_{\lambda}\right\}_{\lambda \in \Lambda}$ are algebraically independent over $k$.

(2) $\left\{d w_{\lambda}\right\}_{\lambda \in \Lambda}$ are linearly independent over $K$ in $\Omega_{K / k}$.

Write $\left\{w_{\Lambda}\right\}$ for $\left\{w_{\lambda}\right\}_{\lambda \in \Lambda}$. Let $\left\{v_{\phi}\right\}_{\phi \in \Phi}$ be a set of elements of $K$ such that $\left\{d w_{\Lambda}\right\} \cup$ $\left\{d v_{\Phi}\right\}$ form a basis for the $K$-vector space $\Omega_{K / k}$. Let $K_{\Lambda}:=k\left(w_{\Lambda}\right)$ and let $K_{\Phi}$ denote the subfield of $K$ generated by $v_{\Phi}$ over $K_{\Lambda}$. We get a decomposition of the extension $k \rightarrow K$ :

$$
k \stackrel{\alpha}{\longrightarrow} K_{\Lambda} \stackrel{\beta}{\longrightarrow} K_{\Phi} \stackrel{\gamma}{\longrightarrow} K,
$$

where $\alpha$ is purely transcendental, $\beta$ is an inseparable algebraic extension and $\gamma$ is unramified (i.e. $\Omega_{K / K_{\Phi}}=0$ ). 
Remark 6.4. In the case when $K$ is finitely generated over $K_{\Lambda}$ (in particular, whenever $K$ is finitely generated over $k$ ), we have some extra information about the size of $\Phi$ and $\Omega_{K / K_{\Lambda}}$. First of all, in this case we have $\# \Phi<\infty$. Secondly, $K$ must be algebraic over $K_{\Lambda}$, otherwise we could enlarge the set $\Lambda$ (cf. Remark 6.3), and this would contradict the fact that $w_{\Lambda}$ is the maximal set satisfying conditions (1) and (2). If $K$ is finitely generated over $K_{\Phi}$, then $K$ is separable algebraic over $K_{\Phi}$, since $\Omega_{K / K_{\Phi}}=0$ (cf. Remark 6.3).

Next, we prove a generalization of the primitive element theorem, which deals with the minimal number of generators of a finitely generated field extension.

Theorem 6.5. Let $\delta: k \rightarrow K$ be a finitely generated field extension. Let $d:=$ $\operatorname{dim}_{K} \Omega_{K / k}$. Then the minimal number of generators of $K$ over $k$ is equal to $d+1$ if $\delta$ is separable but not pure transcendental, and to $d$ otherwise.

Proof. Consider a decomposition (6.1) of $\delta$. Let $t:=\operatorname{tr}$. $\operatorname{deg} K / k$, so that $t=\# \Lambda$ and $d=t+\# \Phi$ (cf. Remark 6.3). First, suppose $\delta$ is separable. In this case $t=d$. Then the minimal number of generators of $K$ is at least $d$. If $K$ can be generated by exactly $d$ elements, then it is pure transcendental over $k$. Otherwise $K$ is generated by one element over $K_{\Lambda}$ by the primitive element theorem, hence the minimal number of generators of $K$ over $k$ is $d+1$. This proves the theorem in the separable case.

Suppose $\delta$ is not separable. It is obvious that $K$ cannot be generated by fewer than $d$ elements. Let us prove that $d$ elements are enough. Since $K_{\Lambda}$ is generated over $k$ by $t$ elements and $\operatorname{dim}_{K} \Omega_{K / K_{\Lambda}}=d-t$, we may replace $k$ by $K_{\Lambda}$. In other words, we may assume that $K$ is algebraic over $k$. Our proof is by induction on $d$. First, let $d=1$. Let $k_{s}$ be the separable closure of $k$ in $K$ and let $v$ be any element of $K$ such that $d v$ generates $\Omega_{K / k} \cong \Omega_{K / k_{s}}$. Then $K=k_{s}(v) K^{p}$ by Corollary 6.2, hence $K=k_{s}(v) K^{p^{n}}$ for all $n$, so that $K=k_{s}(v)$. Now, it is well known and easy to prove that a composition of a separable algebraic extension with a simple algebraic extension is again simple [9, §VII.6, Theorem 14, p. 185 and Exercise 4, p. 190]. The case $d=1$ is proved.

Next, let $d>1$. Let $v_{1}, \ldots, v_{d}$ be a set of elements of $K$ which induce a basis of $\Omega_{K / k}$. We have $K=k_{s}\left(v_{1}, \ldots, v_{d}\right) K^{p}$, hence $K=k_{s}\left(v_{1}, \ldots, v_{d}\right) K^{p^{n}}$ for all $n$, hence $K=k_{s}\left(v_{1}, \ldots, v_{d}\right)$. Now, $k_{s}\left(v_{1}\right)$ is a simple extension of $k$ by the $d=1$ case, and $K=k_{s}\left(v_{1}, \ldots, v_{d}\right)$ is generated over $k_{s}\left(v_{1}\right)$ by $d-1$ elements, hence $K$ is generated over $k$ by $d$ elements, as desired.

Remark 6.6. Another way to phrase Theorem 6.5 is that if $\sigma: k \rightarrow K$ is an inseparable finitely generated field extension, then the set $v_{\Phi}$ of (6.1) can always be chosen in such a way that $K=K_{\Phi}$. Indeed, if $\sigma$ is inseparable and finitely generated, Theorem 6.5 says that the smallest number of generators of $K$ over $K_{\Lambda}$ is $\# \Phi$, hence we may choose $v_{\Phi}$ so that $K=K_{\Phi} \equiv K_{\Lambda}\left(v_{\Phi}\right)$.

Let $V_{\phi}, \phi \in \Phi$, be independent variables. Let $I$ denote the kernel of the map $K_{\Lambda}\left[V_{\Phi}\right] \rightarrow K_{\Phi}$ which sends $V_{\phi}$ to $v_{\phi}$. Choose a well-ordering of $\Phi$. For an element $\phi \in \Phi$, define $\Phi_{\phi}:=\left\{\phi^{\prime} \in \Phi \mid \phi^{\prime}<\phi\right\}$. Let $K_{\phi}$ denote the subfield of $K_{\Phi}$ generated by $v_{\Phi_{\phi}}$ over $K_{\Lambda}$. Let $g_{\phi} \in K_{\phi}\left[V_{\phi}\right]$ denote the (monic) minimal polynomial of $v_{\phi}$ over $K_{\phi}$. Pick and fix a representative $G_{\phi}$ of $g_{\phi}$ in $K_{\Lambda}\left[V_{\Phi_{\phi}}\right]\left[V_{\phi}\right]$. Since $\frac{K_{\Lambda}\left[V_{\Phi}\right]}{\left(G_{\Phi}\right)}$ is a field, the relations $G_{\Phi}$ form a set of generators of $I$. By construction, the elements $G_{\Phi}$ 
form a minimal set of generators of $I$, hence they induce a basis of the $K_{\Phi}$-vector space $\frac{I}{I^{2}}$.

Lemma 6.7. We have a natural isomorphism $H_{1}\left(k, K_{\Phi}, K_{\Phi}\right) \cong \frac{I}{I^{2}}$. In particular, the set $\Phi$ has the same cardinality as any basis of the $K_{\Phi}$-vector space $H_{1}\left(k, K_{\Phi}, K_{\Phi}\right)$.

Proof. By definition, the elements $\left\{d w_{\Lambda}\right\} \cup\left\{d v_{\Phi}\right\}$ are $K$-linearly independent in $\Omega_{K / k}$. Since there is a natural homomorphism $\Omega_{K_{\Phi}} \otimes_{K_{\Phi}} K \rightarrow \Omega_{K / k},\left\{d w_{\Lambda}\right\} \cup\left\{d v_{\Phi}\right\}$ are also $K_{\Phi}$-linearly independent in $\Omega_{K_{\Phi}}$. Since $\left\{d w_{\Lambda}\right\} \cup\left\{d v_{\Phi}\right\}$ generate $K_{\Phi}$ as a field over $k$, we have

$$
\Omega_{K_{\Phi} / k}=\left(\bigoplus_{\lambda \in \Lambda} K_{\Phi} d w_{\lambda}\right) \bigoplus\left(\bigoplus_{\phi \in \Phi} K_{\Phi} d v_{\phi}\right) .
$$

The ring $K_{\Lambda}\left[V_{\Phi}\right]$ is a localization of a polynomial ring over $k$, hence

$$
H_{1}\left(k, K_{\Lambda}\left[V_{\Phi}\right], K_{\Phi}\right)=0 .
$$

By (6.2), (6.3) and Property 2.2 (applied to the surjective map $K_{\Lambda}\left[V_{\Phi}\right] \rightarrow K_{\Phi}$ ), the Jacobi-Zariski sequence $(2.5)$ for the triple $k \rightarrow K_{\Lambda}\left[V_{\Phi}\right] \stackrel{s}{\rightarrow} K_{\Phi}$ takes the form

$$
\begin{aligned}
0 \rightarrow H_{1}\left(k, K_{\Phi}, K_{\Phi}\right) \rightarrow \frac{I}{I^{2}} & \rightarrow \Omega_{K_{\Lambda}\left[V_{\Phi}\right] / k} \otimes K_{\Phi} \\
& \stackrel{d s}{\longrightarrow}\left(\bigoplus_{\lambda \in \Lambda} K_{\Phi} d w_{\lambda}\right) \bigoplus\left(\bigoplus_{\phi \in \Phi} K_{\Phi} d v_{\phi}\right) \rightarrow 0 .
\end{aligned}
$$

Since $d s$ is an isomorphism, (6.4) implies that $H_{1}\left(k, K_{\Phi}, K_{\Phi}\right) \cong \frac{I}{I^{2}}$, as desired.

Corollary 6.8. Let $x_{1}, \ldots, x_{a}$ be elements of $I$. The elements $x_{1}, \ldots, x_{a}$ form a regular system of parameters of the regular local ring $K_{\Lambda}\left[V_{\Phi}\right]_{I}$ if and only if the natural images of $x_{1}, \ldots, x_{a}$ in $H_{1}\left(k, K_{\Phi}, K_{\Phi}\right)$ under the isomorphism of Lemma 6.7 form a basis of $H_{1}\left(k, K_{\Phi}, K_{\Phi}\right)$.

Lemma 6.9. There is a natural injection $\iota: H_{1}\left(k, K_{\Phi}, K\right) \rightarrow H_{1}(k, K, K)$. If $K$ is separable over $K_{\Phi}$ (cf. Remark 6.4), then $\iota$ is an isomorphism.

Proof. Immediate from the Jacobi-Zariski sequence for the triple $k \rightarrow K_{\Phi} \rightarrow K$ (Property 2.18): $0 \rightarrow H_{1}\left(k, K_{\Phi}, K\right) \rightarrow H_{1}(k, K, K) \rightarrow H_{1}\left(K_{\Phi}, K, K\right)$, and Property 2.19 .

Corollary 6.10. Keep the above notation. Suppose $\operatorname{dim}_{K} H_{1}(k, K, K)<\infty$. Then $\Phi$ is a finite set and $\# \Phi \leq \operatorname{dim} H_{1}(k, K, K)$. If, in addition, $K$ is separable over $K_{\Phi}$, then $\# \Phi=\operatorname{dim} H_{1}(k, K, K)$.

Proof. Immediate from Lemmas 6.7 and 6.9.

Lemma 6.11. Let $k \rightarrow K$ be a field extension. Assume that $\operatorname{dim} H_{1}(k, K, K)<\infty$. Then there exists a subfield $L \subset K$, containing $k$ and finitely generated over $k$, such that the natural map $H_{1}(k, L, K) \rightarrow H_{1}(k, K, K)$ is an isomorphism. Fix one such $L$. Then for any subfield $K^{\prime} \subset K$ with $L \subset K^{\prime}$, the natural map $H_{1}\left(k, K^{\prime}, K\right) \rightarrow$ $H_{1}(k, K, K)$ is an isomorphism. Finally, let $K_{0}$ be an extension of $k$, contained in $K^{\prime}$, such that the natural map $\Omega_{K_{0} / k} \otimes_{K_{0}} K \rightarrow \Omega_{K / k}$ is injective. Then the natural map $H_{1}\left(K_{0}, K^{\prime}, K\right) \rightarrow H_{1}\left(K_{0}, K, K\right)$ is an isomorphism. 
Proof. Write $K$ as a filtered inductive limit of its subfields which are finitely generated over $k: K=\underset{\vec{i}}{\lim _{i}} K_{i}$. Since André homology commutes with direct limits ([18, Lemma 3.2] and [2, Chapter III, Proposition 35]), $H_{1}(k, K, K)=\underset{\vec{i}}{\lim _{1}} H_{1}\left(k, K_{i}, K\right)$. Hence there exists a subfield $L \subset K$, finitely generated over $k$, with $H_{1}(k, L, K) \cong$ $H_{1}(k, K, K)$. For any field $K^{\prime}$ such that $L \subset K^{\prime} \subset K$, the map $H_{1}(k, L, K) \rightarrow$ $H_{1}(k, K, K)$ factors through $H_{1}\left(k, K^{\prime}, K\right)$, so $H_{1}\left(k, K^{\prime}, K\right) \rightarrow H_{1}(k, K, K)$ is surjective. The injectivity of $H_{1}\left(k, K^{\prime}, K\right) \rightarrow H_{1}(k, K, K)$ is given by the JacobiZariski sequence for $k \rightarrow K^{\prime} \rightarrow K$ (Property 2.18), so

$$
H_{1}\left(k, K^{\prime}, K\right) \cong H_{1}(k, K, K),
$$

as desired. To prove the last statement of the lemma we first note that the map $\Omega_{K_{0} / k} \otimes_{K_{0}} K \rightarrow \Omega_{K / k}$ factors through $\Omega_{K^{\prime} / k} \otimes_{K^{\prime}} K \rightarrow \Omega_{K / k}$; this implies that the map $\Omega_{K_{0} / k} \otimes_{K_{0}} K \rightarrow \Omega_{K^{\prime} / k} \otimes_{K^{\prime}} K$ is also injective. Now the last statement of the lemma follows from (6.5) and the commutative diagram

$$
\begin{aligned}
& H_{1}\left(k, K_{0}, K\right) \rightarrow H_{1}\left(k, K^{\prime}, K\right) \rightarrow H_{1}\left(K_{0}, K^{\prime}, K\right) \rightarrow \Omega_{K_{0} / k} \otimes K \rightarrow \Omega_{K^{\prime} / k} \otimes K \\
& \| \quad \text { ॥ }\|\quad \downarrow \quad\| \quad \downarrow \\
& H_{1}\left(k, K_{0}, K\right) \rightarrow H_{1}(k, K, K) \rightarrow H_{1}\left(K_{0}, K, K\right) \rightarrow \Omega_{K_{0} / k} \otimes K \rightarrow \quad \Omega_{K / k}
\end{aligned}
$$

given by the Jacobi-Zariski sequences for the triples $k \rightarrow K_{0} \rightarrow K^{\prime}$ and $k \rightarrow K_{0} \rightarrow$ $K$, by the five lemma.

Lemma 6.12. Let $k \rightarrow K_{0} \rightarrow K$ be a composition of field extensions. Assume that the natural map $\Omega_{K_{0} / k} \otimes_{K_{0}} K \rightarrow \Omega_{K / k}$ is injective and the natural injection $H_{1}\left(k, K_{0}, K\right) \rightarrow H_{1}(k, K, K)$ is an isomorphism. Then $K$ is separable over $K_{0}$.

Proof. Immediate from the Jacobi-Zariski sequence for the triple $k \rightarrow K_{0} \rightarrow K$ and Property 2.19.

Let $\sigma: k \rightarrow K$ be a field extension with $\operatorname{dim} H_{1}(k, K, K)<\infty$. Consider a decomposition of $\sigma$ of the form (6.1). Although $K$ is unramified over $K_{\Phi}$, there need not, in general, exist a finitely generated extension of $K_{\Phi}$, contained in $K$, over which $K$ is separable. For the proof of Theorem 1.2 we will need to find a decomposition $k \rightarrow \tilde{K} \rightarrow K$ of $\sigma$ such that $K$ is separable over $\tilde{K}$ and such that $\tilde{K}$ is the limit of an ascending sequence of finitely generated extensions of $K_{\Phi}$, contained in $K$.

Proposition 6.13. Let $\sigma: k \rightarrow K$ be a field extension such that $\operatorname{dim} H_{1}(k, K, K)<$ $\infty$. Consider a decomposition of $\sigma$ of the form (6.1). There exists a sequence $K_{1} \rightarrow K_{2} \rightarrow \cdots \rightarrow K_{n} \rightarrow \cdots$ of finitely generated extensions of $K_{\Phi}$ contained in $K$, having the following properties.

(1) For each $i \in \mathbb{N}$, the natural map $H_{1}\left(k, K_{i}, K\right) \rightarrow H_{1}(k, K, K)$ is an isomorphism.

(2) Let $\tilde{K}:=\lim _{i \rightarrow \infty} K_{i}$. Then $K$ is étale over $\tilde{K}$.

Proof. If $K$ is separable over $K_{\Phi}$, put $K_{i}=\tilde{K}=K_{\Phi}$ and there is nothing to prove. Suppose $K$ is not separable over $K_{\Phi}$ (in particular, char $k=p>0$ ). Let $K_{1}$ be a finitely generated extension of $K_{\Phi}$, contained in $K$, such that the natural map $H_{1}\left(k, K_{1}, K\right) \rightarrow H_{1}(k, K, K)$ is an isomorphism ( $K_{1}$ exists by Lemma 6.11$)$. We 
define the $K_{i}$ recursively as follows. Suppose $K_{i}$ is defined. Since $K$ is unramified over $K_{\Phi}$ we have $K=K_{\Phi} K^{p}$ (Corollary 6.2), so $K_{i} \subset K_{\Phi} K^{p}$. Define $K_{i+1}$ to be a finitely generated extension of $K_{i}$, contained in $K$, such that $K_{i} \subset K_{\Phi} K_{i+1}^{p}$. This defines $K_{i}$ for all $i \in \mathbb{N}$. Put $\tilde{K}=\lim _{i \rightarrow \infty} K_{i}$. Now (1) holds by definition of $K_{1}$ and Lemma 6.11. Since $K_{\Phi} \subset \tilde{K}, K$ is unramified over $\tilde{K}$. By construction, $\tilde{K}=K_{\Phi} \tilde{K}^{p}$, so $\tilde{K}$ is unramified over $K_{\Phi}$ (Corollary 6.2 ). Then the Jacobi-Zariski sequence shows that the natural map $\Omega_{K_{\Phi} / k} \otimes_{K_{\Phi}} \tilde{K} \rightarrow \Omega_{\tilde{K} / k}$ is surjective; hence so is the map $\Omega_{K_{\Phi} / k} \otimes_{K_{\Phi}} K \rightarrow \Omega_{\tilde{K} / k} \otimes_{\tilde{K}} K$. Since the inclusion $\Omega_{K_{\Phi} / k} \otimes_{K_{\Phi}} K \hookrightarrow \Omega_{K / k}$ factors through $\Omega_{\tilde{K} / k} \otimes_{\tilde{K}} K$, the natural map $\Omega_{\tilde{K} / k} \otimes_{\tilde{K}} K \rightarrow \Omega_{K / k}$ is injective. The map $H_{1}(k, \tilde{K}, K) \rightarrow H_{1}(k, K, K)$ is an isomorphism by Lemma 6.11. Thus $K$ is separable over $\tilde{K}$ by Lemma 6.12 . Combining this with the fact that $K$ is unramified over $\tilde{K}$, we get that $K$ is étale over $\tilde{K}$ by Properties 2.4 and 2.19 . This completes the proof.

\section{$\S 7$. RESIDUe FIELD EXTENSIONS \\ INDUCED BY FORMALLY SMOOTH HOMOMORPHISMS}

Let $\sigma:(A, m, k) \rightarrow(B, P, K)$ be a formally smooth local homomorphism of local noetherian rings. Let $\hat{B}$ denote the formal completion of $B$. In this section we deal with the difficulties coming from the inseparability of the field extension $k \rightarrow K$, induced by $\sigma$, by proving the following version of the Nica-Popescu theorem (see Corollary 7.9 for the original Nica-Popescu theorem). We construct a local noetherian $A$-algebra $A^{\bullet}$, smooth over $A$, and a commutative diagram

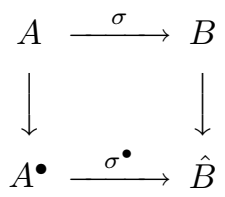

such that $\sigma^{\bullet}$ is formally smooth and induces an isomorphism of the residue field of $A^{\bullet}$ with $K$. We may take $\operatorname{dim} A^{\bullet}=\operatorname{dim} A+\operatorname{dim}_{K} H_{1}(k, K, K)$ (we will see that any ring $A^{\bullet}$ having the above properties must be at least of that dimension). Let $m^{\bullet}$ denote the maximal ideal of $A^{\bullet}$ and let $q:=\operatorname{dim} \frac{\hat{B}}{m^{\bullet} \hat{B}}$. If we adjoin $q$ independent variables to $A^{\bullet}$ and send them to a set of $q$ elements of $B$ inducing a regular system of parameters of $\frac{\hat{B}}{m^{\bullet} \hat{B}}$, the resulting homomorphism will still be formally smooth by Corollary 2.25. In other words, we can always enlarge $A^{\bullet}$ so that $\operatorname{dim} A^{\bullet}=\operatorname{dim} B$. By construction, $A^{\bullet}$ will be a filtered inductive limit of smooth $A$-algebras of finite type. The situation is greatly simplified in the special case when the field extension $k \rightarrow K$ is finitely generated. We will point out what happens in the finitely generated case in order to give the reader an appreciation of the difficulties which arise when $K$ is not finitely generated over $k$ and the need for the somewhat involved construction of $A^{\bullet}$, carried out in this section.

Acknowledgement. The results of this section are closely related to those of [12]. Since both our statements and proofs are somewhat different from those of Nica and Popescu, we prefer to give an independent exposition.

Let $B_{0}:=\frac{B}{m B}$ and let $P_{0}$ denote the maximal ideal of $B_{0}$. Formal smoothness is preserved by base change [10, Chapter 11, (28.E), p. 201]. Taking base change of $\sigma$ 
by $k=\frac{A}{m}$, we see that the local ring $\left(B_{0}, P_{0}, K\right)$ is formally smooth (equivalently, geometrically regular - cf. Proposition 2.6) over $k$. This means that

$$
H_{1}\left(k, B_{0}, W\right)=0 \text { for any } B_{0} \text {-module } W
$$

(Proposition 2.6). Let $d:=\operatorname{dim} B-\operatorname{dim} A=\operatorname{dim} B_{0}$.

Lemma 7.1. We have $\operatorname{dim} H_{1}(k, K, K) \leq d$.

Proof. Immediate from the Jacobi-Zariski sequence (2.5) for the triple $k \rightarrow B_{0} \rightarrow$ $K$, Property 2.2 and (7.1) (with $W=K): 0 \rightarrow H_{1}(k, K, K) \rightarrow \frac{P_{0}}{P_{0}^{2}}$.

Consider the residue field extension $k \rightarrow K$, induced by $\sigma$. Let $K_{\Lambda}$ and $K_{\Phi}$ be as in (6.1). Let $K_{1}$ be a finitely generated extension of $K_{\Phi}$, contained in $K$, such that the natural map

$$
H_{1}\left(k, K_{1}, K\right) \rightarrow H_{1}(k, K, K)
$$

is an isomorphism ( $K_{1}$ exists by Lemma 6.11). Pick a basis for $\Omega_{K_{1} / k}$ of the form $\left\{d w_{\Lambda_{1}}\right\} \cup\left\{d v_{\Phi_{1}}\right\}$ such that $\Lambda_{1} \supset \Lambda, \Phi_{1} \supset \Phi$, the sets $\Lambda_{1} \backslash \Lambda$ and $\Phi_{1}$ are finite and $w_{\Lambda_{1}}$ are algebraically independent over $k$, while $v_{\Phi_{1}}$ are algebraic over $K_{\Lambda_{1}}=k\left(w_{\Lambda_{1}}\right)$. Note that if $K$ is finitely generated over $k$, we may take $K_{1}=K_{\Phi}=K, \Lambda_{1}=\Lambda$ and $\Phi_{1}=\Phi$ (cf. Remarks 6.4 and 6.6 and Lemma 6.9). We get a decomposition (6.1) for the field extension $k \rightarrow K_{1}: k \rightarrow K_{\Lambda_{1}} \rightarrow K_{\Phi_{1}} \rightarrow K_{1}$. Since $K_{1}$ is finitely generated over $K_{\Phi_{1}}$ and since $\Omega_{K / K_{\Phi_{1}}}=0, K_{1}$ is separable over $K_{\Phi_{1}}$ (cf. Remarks 6.3 and 6.4). By Lemma 6.9, we get an isomorphism

$$
H_{1}\left(k, K_{\Phi_{1}}, K_{1}\right) \rightarrow H_{1}\left(k, K_{1}, K_{1}\right) .
$$

Let $a=\# \Phi_{1}$. By Corollary 6.10, applied to the field extension $k \rightarrow K_{1}$, and using the fact that $K$ is flat over $K_{1}$, we obtain $a=\operatorname{dim}_{K_{1}} H_{1}\left(k, K_{1}, K_{1}\right)=$ $\operatorname{dim}_{K} H_{1}\left(k, K_{1}, K\right)=\operatorname{dim}_{K} H_{1}(k, K, K)$. Make the identification $\Phi_{1}=\{1, \ldots, a\}$. For $\lambda \in \Lambda_{1}$, let $\mathbf{w}_{\lambda}$ be any representative of $w_{\lambda}$ in $B$; similarly for $\mathbf{v}_{i}, i \in \Phi_{1}$. Let $V_{\Phi_{1}}=\left(V_{1}, \ldots, V_{a}\right)$ be independent variables. Write $K_{\Phi_{1}} \cong \frac{K_{\Lambda_{1}}\left[V_{\Phi_{1}}\right]}{I}$ and let $\left(G_{1}, \ldots, G_{a}\right)$ be a base of $I$, constructed in $\S 6$ (with $\Phi$ replaced by $\Phi_{1}$ ), so that $G_{i} \in K_{\Lambda_{1}}\left[V_{1}, \ldots, V_{i}\right]$. Let $\mathbf{G}_{i}, 1 \leq i \leq a$, be the representative of $G_{i}$ in $B\left[V_{i}\right]$ obtained by replacing $w_{\lambda}$ by $\mathbf{w}_{\lambda}$ and $v_{j}$ by $\mathbf{v}_{j}, j<i$. For $1 \leq i \leq a$, let $x_{i}:=\mathbf{G}_{i}\left(\mathbf{v}_{i}\right)$. Let $A_{1}:=A\left[W_{\Lambda_{1}}, V_{\Phi_{1}}\right]_{P \cap A\left[W_{\Lambda_{1}}, V_{\Phi_{1}}\right]}$.

Theorem 7.2. (1) The elements $x_{1}, \ldots, x_{a}$ can be extended to a regular system of parameters of $B_{0}$.

(2) Let $W_{\Lambda_{1}}=\left\{W_{\lambda} \mid \lambda \in \Lambda_{1}\right\}$ be independent variables and consider the map $A_{1} \rightarrow B$ which sends $W_{\lambda}$ to $\mathbf{w}_{\lambda}$ and $V_{i}$ to $\mathbf{v}_{i}$. This map is injective and flat.

Proof. (1) Consider the homomorphism between the triples $k \rightarrow K_{\Lambda_{1}}\left[V_{\Phi_{1}}\right] \rightarrow K_{\Phi_{1}}$ and $k \rightarrow B_{0} \rightarrow K$ (the map $K_{\Lambda_{1}}\left[V_{\Phi_{1}}\right] \rightarrow B_{0}$ is given by sending $W_{\lambda}$ to $\mathbf{w}_{\lambda}$ and $V_{i}$ to $\mathbf{v}_{i}$ ). By functoriality of André homology, we obtain a commutative diagram of the Jacobi-Zariski sequences:

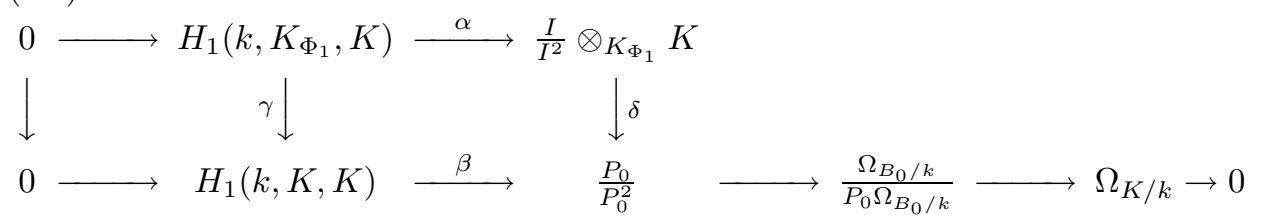


(here the top row is a part of the Jacobi-Zariski sequence for the triple $k \rightarrow$ $K_{\Lambda_{1}}\left[V_{\Phi_{1}}\right] \rightarrow K_{\Phi_{1}}$ and the $K_{\Phi_{1}}$-module $K$, and the bottom row is the Jacobi-Zariski sequence for the triple $\left.k \rightarrow B_{0} \rightarrow K\right)$. Now, $\alpha$ is an isomorphism by Lemma 6.7. The map $\gamma$ is the composition of the isomorphism $H_{1}\left(k, K_{\Phi_{1}}, K\right) \cong H_{1}\left(k, K_{1}, K\right)$ (obtained from (7.3) by tensoring by $K$ ) with the isomorphism (7.2) (this follows by the functoriality of André homology); thus $\gamma$ is an isomorphism. Therefore $\delta$ is injective. The elements $\delta\left(G_{i}\right)$ are nothing but the images of the $x_{i}$ in $\frac{P_{0}}{P_{0}^{2}}$. Since $G_{1}, \ldots, G_{a}$ are $K_{\Phi_{1}}$-linearly independent in $\frac{I}{I^{2}}$, they are $K$-linearly independent in $\frac{I}{I^{2}} \otimes_{K_{\Phi_{1}}} K$, hence $x_{1}, \ldots, x_{a}$ are $K$-linearly independent in $\frac{P_{0}}{P_{0}^{2}}$. (1) is proved. Now (2) follows from Corollary 6.8 and Corollary 2.22. This completes the proof of Theorem 7.2.

Remark 7.3. Suppose that $K$ is finitely generated over $k$. In that case, $K=K_{\Phi_{1}}$ and we may take $A^{\bullet}=A_{1}$; the $A$-algebra $A^{\bullet}$ described in the beginning of this section is already constructed (notice that $A^{\bullet}$ is already in $B$; there is no need to pass to completions).

We continue with our construction of $A^{\bullet}$ in the general case.

Definition 7.4. Let $A^{\prime}$ be a noetherian $A_{1}$-subalgebra of $\hat{B}$ such that $\operatorname{dim} A^{\prime}=$ $\operatorname{dim} A+a$. Let $x_{1}, \ldots, x_{a}$ be as in Theorem 7.2. We say that $A^{\prime}$ is unramified over $A_{1}$ if $A^{\prime}$ is flat over $A$ and $\frac{A^{\prime}}{m A^{\prime}}$ is a regular local ring of dimension $a$ with regular parameters $x_{1}, \ldots, x_{a}$.

If $A^{\prime}$ is unramified over $A_{1}$, then the inclusion $A^{\prime} \rightarrow \hat{B}$ is flat by Corollary 2.22 . Also, $A_{1}$ is unramified over itself by definition of $x_{1}, \ldots, x_{a}$.

Proposition 7.5. Let $\left(A_{0}, P_{0}, K_{0}\right)$ and $\left(A_{2}, P_{2}, K_{2}\right)$ be two local noetherian

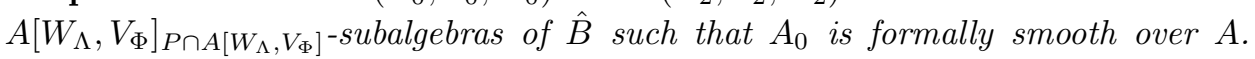
Assume that there exists a non-negative integer $b \leq a$ such that $x_{1}, \ldots, x_{b}$ induce a regular system of parameters of $\frac{A_{0}}{m A_{0}}$. Assume that the inclusion $A_{0} \rightarrow \hat{B}$ is a formally smooth homomorphism of local rings and that $A_{2}$ contains $A_{1}$ and is unramified over it (in particular, $\operatorname{dim} A_{2}=\operatorname{dim} A+a$ ). Assume that the map $\Omega_{K_{0} / k} \otimes_{K_{0}} K \rightarrow \Omega_{K / k}$ is injective. Finally, assume that char $K=p>0$, that $K_{2}$ is either inseparable or purely transcendental over $K_{0}$ and that $A_{2}$ is a localization of a polynomial ring over $A_{0}$ (in finitely many variables). Then there exists a sequence $A_{2} \rightarrow A_{3} \rightarrow \cdots$ where each $A_{i}$ is a localization of a polynomial ring over $A_{0}$ in finitely many variables, contained in $\hat{B}$, with the following properties:

(1) $\operatorname{dim} A_{i}=\operatorname{dim} A+a$ for all $i \geq 2$.

(2) $A_{i}$ is unramified over $A_{1}$ for all $i \geq 2$.

(3) $\tilde{A}:=\bigcup_{i=1}^{\infty} A_{i}$ is étale over $A_{0}$ (in particular, smooth).

(4) The inclusion $\tilde{A} \rightarrow \hat{B}$ is a formally smooth homomorphism of local rings and the induced residue field extension is étale.

Proof. We construct the $A_{i}$ recursively as follows. Suppose $A_{i}$ is constructed. Let $K_{i}$ denote the residue field of $A_{i}$. Let $K_{0} \rightarrow K_{\Lambda_{i}} \rightarrow K_{\Phi_{i}}=K_{i}$ be the decomposition (6.1) for the extension $K_{0} \rightarrow K_{i}$, where we can choose $K_{\Phi_{i}}=K_{i}$ by Theorem 6.5 and Remark 6.6. Here $\Lambda_{i}$ and $\Phi_{i}$ are finite sets and $\# \Phi_{i}=\operatorname{dim}_{K_{i}} H_{1}\left(K_{0}, K_{i}, K_{i}\right)=$ $\operatorname{dim}_{K} H_{1}\left(K_{0}, K_{i}, K\right)=\operatorname{dim}_{K} H_{1}\left(K_{0}, K, K\right)$ is independent of $i$ (by Lemma 6.11); in fact, diagram (6.6) shows that $\# \Phi_{i}=a-\operatorname{dim}_{K_{0}} H_{1}\left(k, K_{0}, K_{0}\right)$. Let $w_{\Lambda_{i}}$ denote 
the generators of $K_{\Lambda_{i}}$ over $K_{0}$ and $v_{\Phi_{i}}$ the generators of $K_{i}$ over $K_{\Lambda_{i}}$. We will assume, inductively, that $A_{i}$ is the localization of the polynomial ring $A_{0}\left[W_{\Lambda_{i}}, V_{\Phi_{i}}\right]$ at the prime ideal $P_{i}$ which is, by definition, the kernel of the map $A_{0}\left[W_{\Lambda_{i}}, V_{\Phi_{i}}\right] \rightarrow$ $K_{i}$ which maps $A_{0}$ to $K_{0}, W_{\lambda}$ to $w_{\lambda}$ and $V_{\phi}$ to $v_{\phi}$. Since $\Omega_{K / K_{0}}=0$, we have $\left\{w_{\Lambda_{i}}, v_{\Phi_{i}}\right\} \subset K^{p} K_{0}$ (Corollary 6.2). Let $K_{i+1}$ be a finitely generated extension of $K_{0}$, contained in $K$, such that

$$
\left\{w_{\Lambda_{i}}, v_{\Phi_{i}}\right\} \subset K_{i+1}^{p} K_{0} .
$$

Consider the decomposition (6.1) $K_{0} \rightarrow K_{\Lambda_{i+1}} \rightarrow K_{\Phi_{i+1}}=K_{i+1}$ of the extension $K_{0} \rightarrow K_{i+1}$ (we may take $K_{\Phi_{i+1}}=K_{i+1}$ by Theorem 6.5 and Remark 6.6). As usual, let $w_{\Lambda_{i+1}}$ be a set of algebraically independent generators of $K_{\Lambda_{i+1}}$ and $v_{\Phi_{i+1}}$ a set of $p$-independent generators of $K_{\Phi_{i+1}}$ over $K_{\Lambda_{i+1}}$. Put $A_{i+1}:=A_{0}\left[W_{\Lambda_{i+1}}, V_{\Phi_{i+1}}\right]_{P_{i+1}}$. By (7.5), for $\lambda \in \Lambda_{i}$, there exists $n_{\lambda} \in \mathbb{N}$, elements $a_{\lambda j} \in K_{0}$ and polynomials $e_{\lambda j}, h_{\lambda j} \in K_{0}\left[W_{\Lambda_{i+1}}, V_{\Phi_{i+1}}\right], 1 \leq j \leq n_{\lambda}$, such that the inclusion $K_{i} \rightarrow K_{i+1}$ is given by

$$
w_{\lambda}=\sum_{j=1}^{n_{\lambda}} a_{\lambda j}\left(\frac{e_{\lambda j}\left(w_{\Lambda_{i+1}}, v_{\Phi_{i+1}}\right)}{h_{\lambda j}\left(w_{\Lambda_{i+1}}, v_{\Phi_{i+1}}\right)}\right)^{p}, \quad \lambda \in \Lambda_{i}
$$

(the existence of expressions (7.6) follows from (7.5) because the field $K_{i+1}^{p} K_{0}$ equals the $K_{0}$-subalgebra of $K_{i+1}$, generated by $K_{i+1}^{p}$ ). We also have the analogous statement for $v_{\phi}, \phi \in \Phi_{i}$; elements $a_{\phi j} \in K_{0}$ and $e_{\phi j}, h_{\phi j} \in K_{0}\left[W_{\Lambda_{i+1}}, V_{\Phi_{i+1}}\right]$ for $\phi \in \Phi_{i}$, are defined in the same way. Now the idea is to use the relations (7.6) to lift the inclusion $K_{i} \rightarrow K_{i+1}$ to an inclusion $A_{i} \rightarrow A_{i+1}$. Pick and fix representatives $E_{\lambda j}, H_{\lambda j} \in A_{0}\left[W_{\Lambda_{i+1}}, V_{\Phi_{i+1}}\right]$ of $e_{\lambda j}$ and $h_{\lambda j}$ and similiarly for $E_{\phi j}$ and $H_{\phi j}$. Let $\mathbf{a}_{\lambda j}$ be a representative of $a_{\lambda j}$ in $A_{0}$, similarly for $\mathbf{a}_{\phi j}$. By construction, $H_{\lambda j}, H_{\phi j} \notin$ $P_{i+1}$. Define the homomorphism $\iota_{i}: A_{i} \rightarrow A_{i+1}$ by

$$
\begin{aligned}
\iota_{i}\left(W_{\lambda}\right) & =\sum_{j=1}^{n_{\lambda}} \mathbf{a}_{\lambda j}\left(\frac{E_{\lambda j}}{H_{\lambda j}}\right)^{p}, \quad \lambda \in \Lambda_{i} \\
\iota_{i}\left(V_{\phi}\right) & =\sum_{j=1}^{n_{\phi}} \mathbf{a}_{\phi j}\left(\frac{E_{\phi j}}{H_{\phi j}}\right)^{p}, \quad \phi \in \Phi_{i} .
\end{aligned}
$$

Applying Theorem $7.2(2)$ with $\Lambda_{1}, \Phi_{1}$ and $B$ replaced by $\Lambda_{i}, \Phi_{i}$ and $\hat{B}$, respectively, we see that $A_{i} \subset \hat{B}$ and $\hat{B}$ is flat (hence faithfully flat) over $A_{i}$ for all $i$. This also proves that all the maps $A_{i} \rightarrow A_{i+1}$ are injective, and that $\tilde{A}$ is an $A$-subalgebra of $\hat{B}$.

Lemma 7.6. The $A_{0}$-algebra $\tilde{A}:=\bigcup_{i=2}^{\infty} A_{i}$ is étale over $A_{0}$.

Proof. Consider a commutative diagram

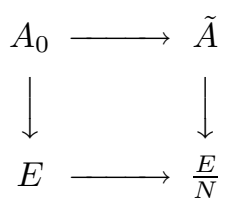

where $E$ is an $A_{0}$-algebra and $N$ is an ideal of $E$ such that $N^{2}=0$. We want to show that there is a unique lifting $\tilde{A} \rightarrow E$ which makes this diagram commutative. It is sufficient to prove that for each $i \geq 2$ there exists a unique lifting 
$\tau_{i}: A_{i} \rightarrow E$ compatible with (7.8). To construct $\tau_{i}$, take any homomorphism $\tau_{i+1}^{\prime}: A_{0}\left[W_{\Lambda_{i+1}}, V_{\Phi_{i+1}}\right] \rightarrow E$, compatible with (7.8). Since

$$
A_{i} \subset A_{0} A_{i+1}^{p}
$$

by (7.7) and since $N^{p}=N^{2}=0, \tau_{i+1}^{\prime}$ determines a unique lifting $\tau_{i}: A_{i} \rightarrow E$.

Taking $E=\frac{B}{P^{j+1}}$ and $N=\frac{P^{j}}{P^{j+1}}$ in (7.8) and passing to the limit as $j \rightarrow \infty$ shows that there exists a unique extension of the homomorphism $A_{0} \rightarrow \hat{B}$ to $\tilde{A} \rightarrow \hat{B}$. We have $\Omega_{\tilde{A} / A_{0}}=0$ by (7.9). The map $A_{0} \rightarrow \hat{B}$ is formally smooth, hence the inclusion $\tilde{A} \rightarrow \hat{B}$ is formally smooth (by Proposition 2.6 and Remark 2.10). Let $\tilde{K}$ denote the residue field of $\tilde{A}$. Since $K_{1} \subset \tilde{K}$, we have

$$
H_{1}(k, \tilde{K}, K) \cong H_{1}(k, K, K)
$$

by Lemma 6.11. By Lemma $6.12, K$ is separable over $\tilde{K}$. Since $K_{\Phi} \subset \tilde{K}$ and $\Omega_{K / K_{\Phi}}=0$, we have $\Omega_{K / \tilde{K}}=0$ by the Jacobi-Zariski sequence. Hence $K$ is étale over $\tilde{K}$ (Properties 2.4 and 2.19), as desired.

Next, we show that $\tilde{A}$ is noetherian. Indeed, all the maps $A_{i} \rightarrow \hat{B}$ are faithfully flat. Thus the noetherianity of $\tilde{A}$ is given by the following lemma.

Lemma 7.7. Let $\left\{A_{i}\right\}$ be an inductive system of noetherian rings together with a faithfully flat map from each $A_{i}$ to a fixed noetherian ring $B$. Then $\underset{\vec{i}}{\lim } A_{i}$ is again noetherian.

Proof. Let $I_{j}, j \in \mathbb{N}$ be an ascending chain of ideals of $\tilde{A}$. Then the chain $I_{j} B$ stabilizes, say for $j \geq j_{0}$. The ideal $I_{j_{0}} B$ is finitely generated, hence there exists $i$ sufficiently large so that $I_{j_{0}} B=\left(I_{j_{0}} \cap A_{i}\right) B$. Then for any $i^{\prime}>i$ and any $j \geq j_{0}$ we have $I_{j} \cap A_{i^{\prime}} \subset\left(I_{j} B\right) \cap A_{i^{\prime}}=\left(I_{j_{0}} B\right) \cap A_{i^{\prime}}=\left(I_{j_{0}} \cap A_{i}\right) B \cap A_{i^{\prime}} \subset\left(I_{j_{0}} \cap A_{i^{\prime}}\right) B \cap A_{i^{\prime}}=$ $I_{j_{0}} \cap A_{i^{\prime}}$, where the last equality holds by faithful flatness of the map $A_{i^{\prime}} \rightarrow B$. Since this holds for all $i^{\prime}>i$, we have $I_{j}=I_{j_{0}}$ for $j \geq j_{0}$, as desired.

Finally, it remains to check that each $A_{i}$ is unramified over $A_{1}$. Since the $A_{i}$ are localizations of polynomial rings over $A_{0}$, they are flat over $A_{0}$, hence also over $A$. It remains to show that $x_{1}, \ldots, x_{a}$ induce a regular system of parameters of $\frac{A_{i}}{m A_{i}}$. It is sufficient to prove that $x_{b+1}, \ldots, x_{a}$ induce a regular system of parameters of $\frac{A_{i}}{m_{0} A_{i}}$. For each $i$, write $\frac{A_{i}}{m_{0} A_{i}}=\frac{K_{0}\left[W_{\Lambda_{i}}, V_{\Phi_{i}}\right]}{I_{i}}$. We proceed by induction on $i$. For $i=2$, our statement is true because $A_{2}$ was assumed to be unramified over $A_{1}$. Suppose the statement is true for $i$. Consider the commutative diagram

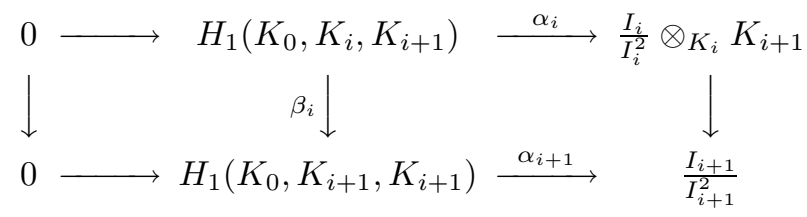

The maps $\alpha_{i}$ and $\alpha_{i+1}$ are isomorphisms by Lemma 6.7, and $\beta_{i}$ is an isomorphism by Lemma 6.11 . By the induction assumption and Corollary 6.8 , the natural images of $x_{b+1}, \ldots, x_{a}$ in $H_{1}\left(K_{0}, K_{i}, K_{i+1}\right) \cong H_{1}\left(K_{0}, K_{i}, K_{i}\right) \otimes_{K_{i}} K_{i+1}$ form a basis of $H_{1}\left(K_{0}, K_{i}, K_{i+1}\right)$. Hence their images in $H_{1}\left(K_{0}, K_{i+1}, K_{i+1}\right)$ form a basis of $H_{1}\left(K_{0}, K_{i+1}, K_{i+1}\right)$. Therefore $x_{b+1}, \ldots, x_{a}$ induce a regular system of parameters of $\frac{A_{i+1}}{m_{0} A_{i+1}}$, as desired. Proposition 7.5 is proved. 
We will now build up the $A$-algebra $A^{\bullet}$ described at the beginning of this section recursively, using transfinite induction on the set of generators of the residue field. We will start with $A_{0}=A\left[W_{\Lambda}, V_{\Phi}\right]_{P \cap A\left[W_{\Lambda}, V_{\Phi}\right]}$ and use Proposition 7.5 repeatedly, until we arrive at the $A$-algebra $A^{\bullet}$ whose residue field is $K$. But first, we must check that $A_{0}=A\left[W_{\Lambda}, V_{\Phi}\right]_{P \cap A\left[W_{\Lambda}, V_{\Phi}\right]}$ satisfies the hypotheses of Proposition 7.5. Namely, we have to check that $\hat{B}$ is formally smooth over $A\left[W_{\Lambda}, V_{\Phi}\right]_{P \cap A\left[W_{\Lambda}, V_{\Phi}\right]}$.

Lemma 7.8. The ring $\hat{B}$ is formally smooth over $A\left[W_{\Lambda}, V_{\Phi}\right]_{P \cap A\left[W_{\Lambda}, V_{\Phi}\right]}$.

Proof. Since $\hat{B}$ is formally smooth over $B$ and $B$ over $A, \hat{B}$ is formally smooth over $A$. Consider the Jacobi-Zariski sequence for the triple $A \rightarrow A\left[W_{\Lambda}, V_{\Phi}\right]_{P \cap A\left[W_{\Lambda}, V_{\Phi}\right]} \rightarrow$ $\hat{B}$ :

$$
\begin{aligned}
0 & \rightarrow H_{1}\left(A\left[W_{\Lambda}, V_{\Phi}\right]_{P \cap A\left[W_{\Lambda}, V_{\Phi}\right]}, \hat{B}, K\right) \\
& \rightarrow\left(\bigoplus_{\lambda \in \Lambda} K d W_{\lambda}\right) \oplus\left(\bigoplus_{\phi \in \Phi} K d W_{\phi}\right) \rightarrow \Omega_{\hat{B} / A} \otimes K .
\end{aligned}
$$

Now, $\left\{d w_{\lambda}\right\}_{\lambda \in \Lambda}$ and $\left\{w_{\phi}\right\}_{\phi \in \Phi}$ are all linearly independent in $\Omega_{\hat{B} / A} \otimes K$ because their natural images in $\Omega_{K / A} \equiv \Omega_{K / k}$ are linearly independent by definition. Hence the last arrow in $(7.11)$ is injective, so $H_{1}\left(A\left[W_{\Lambda}, V_{\Phi}\right]_{P \cap A\left[W_{\Lambda}, V_{\Phi}\right]}, \hat{B}, K\right)=0$ and the lemma is proved.

Corollary 7.9 (the Nica-Popescu theorem [12]). There exists an increasing sequence $A_{i}^{\prime}$ of subrings of $\hat{B}$, each of which is a localization of a polynomial ring in finitely many variables over $A$, such that $\lim _{i \rightarrow \infty} A_{i}^{\prime}$ is a local noetherian ring of the same dimension as $B$, the inclusion $\lim _{i \rightarrow \infty} A_{i}^{\prime} \rightarrow \hat{B}$ is formally smooth and the induced residue field extension is separable.

Proof. Let $A_{0}=A\left[W_{\Lambda}, V_{\Phi}\right]_{P \cap A\left[W_{\Lambda}, V_{\Phi}\right]}$ in Proposition 7.5. Choose an increasing sequence $\Delta_{i}$ of finite subsets of $\Lambda$ such that for each $\phi \in \Phi_{i}, v_{\phi}$ is algebraic over $k\left(w_{\Delta_{i}}, v_{\Phi}, w_{\Lambda_{i}}\right)$. Put

$$
A_{i}^{\prime \prime}:=A\left[W_{\Delta_{i}}, V_{\Phi}, W_{\Lambda_{i}}, V_{\Phi_{i}}\right]_{P \hat{B} \cap A\left[W_{\Delta_{i}}, V_{\Phi}, W_{\Lambda_{i}}, V_{\Phi_{i}}\right.} .
$$

Extend $x_{1}, \ldots, x_{a}$ to a set $x_{1}, \ldots, x_{d}$ which induces a regular system of parameters of $\frac{B}{m B}$ and let $A_{i}^{\prime}:=A_{i}^{\prime \prime}\left[x_{a+1}, \ldots, x_{d}\right]_{P \hat{B} \cap A_{i}^{\prime \prime}\left[x_{a+1}, \ldots, x_{d}\right]}$. Then $\operatorname{dim} A_{i}^{\prime}=\operatorname{dim} B$. The other conclusions are given by Proposition 7.5.

Theorem 7.10. There exists a smooth local noetherian $A\left[W_{\Lambda}, V_{\Phi}\right]_{P \cap A\left[W_{\Lambda}, V_{\Phi}\right]^{-}}$ algebra $A^{\bullet}$, mapping to $\hat{B}$, such that:

(1) $A^{\bullet}$ is étale over $A\left[W_{\Lambda}, V_{\Phi}\right]_{P \cap A\left[W_{\Lambda}, V_{\Phi}\right]}$.

(2) The homomorphism $A^{\bullet} \rightarrow \hat{B}$ is formally smooth and the induced map

$$
\frac{A^{\bullet}}{P \cap A^{\bullet}} \rightarrow \frac{B}{P}
$$

of residue fields is an isomorphism.

(3) $\operatorname{dim} A^{\bullet}=\operatorname{dim} A+a$.

(4) $A^{\bullet}$ is a filtered inductive limit of smooth local $A\left[W_{\Lambda}, V_{\Phi}\right]_{P \cap A\left[W_{\Lambda}, V_{\Phi}\right]}$-algebras $\bar{A}$ essentially of finite type, over which $\hat{B}$ is flat; the elements $x_{1}, \ldots, x_{a}$ form a regular system of parameters for each of the $\frac{\bar{A}}{m}$. 
Proof. We construct $A^{\bullet}$ by transfinite induction, using Proposition 7.5. Let $\mathcal{A}$ denote the set of local noetherian subalgebras $\tilde{A}$ of $\hat{B}$ such that:

(1) $A\left[W_{\Lambda}, V_{\Phi}\right]_{P \cap A\left[W_{\Lambda}, V_{\Phi}\right]} \subset \tilde{A}$.

(2) $\tilde{A}$ is étale over $A\left[W_{\Lambda}, V_{\Phi}\right]_{P \cap A\left[W_{\Lambda}, V_{\Phi}\right]}$.

(3) $\tilde{A}$ is a filtered inductive limit of smooth $A$-algebras $\bar{A}$ essentially of finite type, such that each $\frac{\bar{A}}{m A}$ is a regular $a$-dimensional local ring with regular parameters $x_{1}, \ldots, x_{a}$.

(4) The map $\tilde{A} \rightarrow B$ is formally smooth and the induced residue field extension is étale.

$\mathcal{A}$ is partially ordered by inclusion; $\mathcal{A} \neq \emptyset$ by Proposition 7.5 , applied to

$$
\begin{aligned}
& A_{0}=A\left[W_{\Lambda}, V_{\Phi}\right]_{P \cap A\left[W_{\Lambda}, V_{\Phi}\right]} \text { and } \\
& A_{2}=A_{1} .
\end{aligned}
$$

Given a subset $\left\{A_{\delta} \mid \delta \in \Delta\right\} \subset \mathcal{A}$, which is totally ordered by inclusion, $\bigcup_{\delta \in \Delta} A_{\delta} \in \mathcal{A}$ (again, since each $A_{\delta}$ comes with a faithfully flat map to the noetherian ring $\hat{B}$, noetherianity of $\bigcup A_{\delta}$ is given by Lemma 7.7). By Zorn's lemma, $\mathcal{A}$ contains a $\delta \in \Delta$

maximal element $\left(A^{\bullet}, m^{\bullet}, K^{\bullet}\right)$. It remains to prove that $K^{\bullet} \cong K$. Suppose not. Take an element $t \in K \backslash K^{\bullet}$. By assumption, $K$ is separable over $K^{\bullet}$. Hence $t$ is either transcendental or separable algebraic over $K^{\bullet}$.

Case 1. $t$ is separable algebraic over $K^{\bullet}$. Let $h$ denote the minimal polynomial of $t$ over $K^{\bullet}$ and let $H$ be any lifting of $h$ to a polynomial over $A^{\bullet}$. Put $\tilde{A}:=\frac{A^{\bullet}[T]}{(H)}$. Since $H$ is separable, the inclusion $A^{\bullet} \rightarrow \hat{B}$ extends in a unique way to a map $\tilde{A} \rightarrow \hat{B}$ (by the implicit function theorem). It is immediate to verify that $\tilde{A} \in \mathcal{A}$, which contradicts the maximality of $A^{\bullet}$.

Case 2. $t$ is transcendental over $K^{\bullet}$. Let $\mathbf{t}$ be any representative of $t$ in $B$. Then $\mathbf{t}$ is transcendental over $A^{\bullet}$ by Corollary 2.22, applied to the triple $A^{\bullet} \rightarrow$ $A^{\bullet}[\mathbf{t}]_{P \cap A} \bullet[\mathbf{t}] \rightarrow \hat{B}$. Let $A_{2}:=A^{\bullet}[\mathbf{t}]_{P \cap A} \bullet[\mathbf{t}]$.

The homomorphism $A^{\bullet} \rightarrow A_{2}$ satisfies the hypotheses of Proposition 7.5, so there exists an $A^{\bullet}$-algebra $\tilde{A} \in \mathcal{A}$, containing $A_{2}$. This is a contradiction, hence $K^{\bullet}=K$.

Corollary 7.11. There exists a local noetherian A-algebra $\left(A^{\prime}, m^{\prime}\right)$, contained in $\hat{B}$, smooth over $A$, such that:

(1) $A^{\prime}$ is a filtered inductive limit of smooth finite type A-algebras.

(2) $\hat{B}$ is formally smooth over $A^{\prime}$.

(3) $m^{\prime} B=P$ (in particular, $\operatorname{dim} A^{\prime}=\operatorname{dim} B$ ).

(4) $\frac{A^{\prime}}{m^{\prime}} \cong K$.

Proof. Extend $x_{1}, \ldots, x_{a}$ to a set $x_{1}, \ldots, x_{d}$ which induces a regular system of parameters of $B_{0}$. Put $A^{\prime}:=A^{\bullet}\left[x_{a+1}, \ldots, x_{d}\right]_{P \cap A} \bullet\left[x_{a+1}, \ldots, x_{d}\right]$. The inclusion $\sigma^{\bullet}$ : $A^{\bullet} \rightarrow \hat{B}$ extends to an inclusion $\sigma^{\prime}: A^{\prime} \rightarrow \hat{B} ; \hat{B}$ is formally smooth over $A^{\prime}$ by Corollary 2.25. Let $m^{\prime}$ denote the maximal ideal of $A^{\prime}$. Then $m^{\prime} \hat{B}=P \hat{B}$.

\section{§. Smoothing of an isolated Singularity over a local Ring}

Let the notation be as in Theorem 1.5 and let $\hat{B}$ denote the $P$-adic completion of $B_{P}$. Combining the results of $\S \S 4-7$, we obtain a commutative diagram (1.7), 
satisfying (1)-(8) of Proposition 1.6, with $B$ replaced by $\hat{B}$ (this is explained in more detail below; it proves Theorems 1.5 and 1.2 in the case when $(B, P)$ is local and $P$-adically complete). In this section we show how to replace $\hat{B}$ by $B_{P}$ (by $P$-adic approximation) and in $\S 9$-how to descend from $B_{P}$ to $B$ (delocalization). We start with two observations pertaining to both this and the next section.

Remark 8.1. Suppose $P=(0)$. Then $B_{P}$ is a field. Then $A_{P \cap A}$ and $\rho_{P}\left(C_{P \cap C}\right)$ are domains. Letting $D=\rho(C)$, we get that $H_{D / A} \neq(0)$, so $D$ satisfies the conclusion of Theorem 1.5 and (1.3). From now on, we will assume that $P \neq(0)$, both in this and the next section.

Let $S=S_{C}\left(\frac{I}{I^{2}}\right)$. As before, we will assume that $H_{S / A} \subset P \cap S$ (otherwise we put $D=S$ and Theorem 1.5 and (1.3) are proved (cf. (5.1)). Then, by (5.1), $H_{S / A} B$ is a minimal prime of $P$. From now on, to simplify the notation, we will replace $C$ by $S$ and assume that there is a presentation $C=\frac{A\left[u_{1}, \ldots, u_{n}\right]}{I}$ such that $\left.\frac{\mathcal{I}}{\mathcal{I}^{2}}\right|_{\text {Spec } C \backslash V\left(H_{C / A}\right)}$ is the trivial vector bundle (cf. Lemma 5.2), both in this section and the next. Note that because of (5.1) and Property 2.16, replacing $C$ by $S$ does not affect condition (3) of Proposition 1.6.

Let $m=P \cap A$. In both this and the next section, we will assume that the homomorphism $\sigma_{P}: A_{m} \rightarrow B_{P}$ is formally smooth in the $P$-adic topology. This is weaker than being regular by Proposition 2.7.

We now state and prove the main result of this section:

Proposition 8.2. Assume that $B$ is local with maximal ideal $P$ (in particular, $\left.P=\sqrt{H_{C / A} B}\right)$. Then there exists a diagram (1.7) satisfying (1)-(8) of Proposition 1.6 (in particular, Theorems 1.5 and 1.2 hold in this case).

Proof. Let $\bar{f}_{1}, \ldots, \bar{f}_{r} \in \Gamma$ (Spec $\left.C \backslash V\left(H_{C / A}\right)\right)$ be sections which freely generate $\left.\frac{\mathcal{I}}{\mathcal{I}^{2}}\right|_{\text {Spec } C \backslash V\left(H_{C / A}\right)}$. Note that this property is preserved after a change of base of the form $\otimes_{A} \bar{A}$, where $\bar{A}$ is essentially of finite type over $A$.

Let $\left(A^{\prime}, m^{\prime}\right)$ be the ring whose existence is asserted in Corollary 7.11 (applied to the formally smooth homomorphism $\sigma_{P}$ ). Apply Proposition 5.1 to the flat homomorphism $\sigma^{\prime}: A^{\prime} \rightarrow \hat{B}$ and the finite type $A^{\prime}$-algebra $C \otimes_{A} A^{\prime}$. We get a commutative diagram (1.7), with $B$ replaced by $\hat{B}$, satisfying (1)-(8) of Proposition 1.6. Moreover, since $A^{\prime}$ is a filtered inductive limit of local $A$-algebras $\bar{A}$, smooth and essentially of finite type, over which both $A^{\prime}$ and $\hat{B}$ are flat (by Corollary 2.22), by Lemma 3.8 we may choose one such $A$-subalgebra $(\bar{A}, \bar{m})$ such that our diagram (1.7) descends to a diagram of $\bar{A}$-algebras, $\bar{m} B=P$ and (4.13) holds for all the generalized blowings up $C_{i}$ of $C \otimes_{A} \bar{A}$ involved in the construction. We obtain a commutative diagram

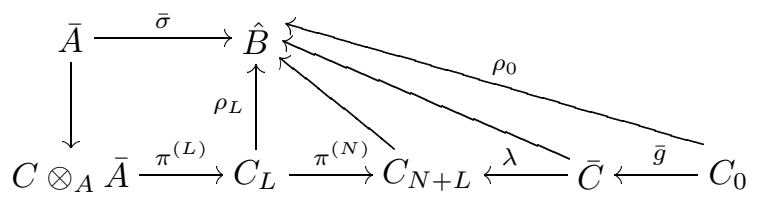

satisfying (1)-(8) of Proposition 1.6. The idea is to approximate (8.1) in the $P$-adic topology to get a diagram (1.7) with $B$ instead of $\hat{B}$. To do this, we will use the following facts, which were proved in the course of the construction of (8.1). 
Properties 8.3. (1) The map $\pi^{(L)}$ is the composition of $L$ generalized blowings up along $\bar{m}$. Here $L=L_{0} L_{1}$, where $L_{0}$ is a positive integer such that

$$
\bar{m}^{L_{0}} B \subset \bar{m} H_{C / A} B
$$

and $L_{1} \in \mathbb{N}$ is such that (5.6) holds.

(2) The algebra $C_{L}$ is an almost complete intersection over $\bar{A}$. Let $C_{L}=\frac{\bar{A}\left[u^{(L)}\right]}{I_{L}}$ be the given presentation of $C_{L}$ and let $f_{1}^{(L)}, \ldots, f_{r}^{(L)} \in I_{L}$ be as in Definition 1.7. The elements $f_{1}^{(L)}, \ldots, f_{r}^{(L)}$ can be chosen so that their respective images in $\Gamma\left(\operatorname{Spec} C_{L} \backslash V\left(m C_{L}\right), \frac{\mathcal{I}_{L}}{\mathcal{I}_{L}^{2}}\right)$ are $y\left(\pi^{(L)}\right)^{*} \bar{f}_{1}, \ldots, y\left(\pi^{(L)}\right)^{*} \bar{f}_{r}$, for some $y \in$ $C_{L} \backslash\left(P \cap C_{L}\right)$ (Lemmas 5.4 and 5.5).

(3) The map $\pi^{(N)}$ is a composition of $N$ generalized blowings up along $\bar{m}$, where $N$ satisfies (1.13) and

$$
\bar{m}^{N} B^{r} \subset J\left(f^{(L)}\right) .
$$

(4) Write

$$
C_{0}=\frac{\bar{A}\left[G_{1}, \ldots, G_{l}\right]}{(F)},
$$

where $F=\left(F_{1}, \ldots, F_{r}\right)$ are linear homogeneous equations over $\bar{A}$. Write $F_{j}=\sum_{i=1}^{l} a_{i j} G_{i}$ and let $a_{j}$ denote the column $r$-vector with entries $a_{i j}$. Let $I(F)$ be the submodule of $A^{r}$ generated by $a_{1}, \ldots, a_{l}$. Then

$$
\bar{m}^{2 N} \subset I(F)
$$

(Lemma 4.5).

(5) Let $K_{\bar{A}}$ denote the kernel of the $\bar{A}$-linear map $\bar{A}^{l} \rightarrow \bar{A}^{r}$ given by the matrix $\left(a_{i j}\right), K_{\hat{B}}$ the kernel of the $\hat{B}$-linear map $\hat{B}^{l} \rightarrow \hat{B}^{r}$ given by the same matrix. Let $\mathbf{g}_{i}=\rho_{0}\left(G_{i}\right) \in \hat{B}$ and let $\mathbf{g}$ denote the l-vector with entries $\mathbf{g}_{i}, 1 \leq i \leq l$. Condition (6) of Proposition 1.6 is equivalent to saying that

$$
\mathrm{g} \in K_{\bar{A}} \hat{B}^{l} .
$$

We now $P$-adically approximate the diagram (8.1). Namely, we will construct a new sequence of generalized blowings up

$$
C \otimes_{A} \tilde{A} \stackrel{\tilde{\pi}_{1}}{\longrightarrow} \tilde{C}_{1} \stackrel{\tilde{\pi}_{2}}{\longrightarrow} \ldots \stackrel{\tilde{\pi}_{N+L}}{\longrightarrow} \tilde{C}_{N+L}
$$

and

$$
\tilde{C}_{0} \stackrel{\tilde{g}}{\rightarrow} \tilde{C} \stackrel{\tilde{\lambda}}{\rightarrow} \tilde{C}_{N+L}
$$

along $\tilde{m}$, where $\tilde{A}$ is, in the sense defined below, a $P$-adic approximation to $\bar{A}$, and (8.7)-(8.8) are $P$-adic approximations to the corresponding maps in (8.1). Let $J$ denote the $\bar{A}$-submodule of $\bar{A}^{r}$ generated by $a_{1}, \ldots, a_{l}$. By (8.5) and Lemma 3.7, $\bar{m}^{t} \bar{A}^{r} \subset J$ for $t \gg 0$. Take $t \in \mathbb{N}$ such that

(1) $P^{t} \hat{B}^{l} \cap K_{\hat{B}} \subset P K_{\hat{B}}$.

(2) $t>2 N$.

Next, choose $t^{\prime} \in \mathbb{N}$ such that

(3) $t^{\prime}>L+2 t$.

(4) $\bar{m}^{t^{\prime}} C_{L}^{n_{L}} \cap \operatorname{Im}\left(d_{L}\right) \subset \bar{m}^{2 t} \operatorname{Im}\left(d_{L}\right)$ 
(in the notation of (5.5)). Here (1) and (4) can be achieved by the Artin-Rees lemma. We will now approximate (8.1) to within $P^{t^{\prime}}$. Condition (1) will be needed to deduce (6) of Proposition 1.6. Condition (2) will be needed to ensure that the hypotheses of Lemma 4.4 hold for the sequence (8.7), and also to prove (7) of Proposition 1.6. (3) will be needed to ensure the hypotheses of Lemma 5.4 and (4) to approximate the elements $f_{1}^{(L)}, \ldots, f_{r}^{(L)} \in I_{L}$ to within $2 t$.

Let $z_{1}, \ldots, z_{k}$ be a set of generators of $\bar{m}$. Since $\bar{A}$ is a local smooth $A$-algebra, we may take $\bar{A}$ to be of the form $\bar{A}=\frac{A[V]_{P \cap A[V]}}{(h)}$, where $V=\left(V_{1}, \ldots, V_{s}\right), h=$ $\left(h_{1}, \ldots, h_{q}\right)$ and

$$
\operatorname{det}\left|\frac{\partial h_{i}}{\partial V_{j}}\right|_{1 \leq i, j \leq q} \bar{A}=\bar{A} .
$$

Let $v_{i}=\bar{\sigma}\left(V_{i}\right)$ (cf. (8.1)). For each $i, 1 \leq i \leq k$, let $\beta_{i}(V) \in A[V]_{P \cap A[V]}$ be a representative of $z_{i}$. Without loss of generality, we may assume that $\beta_{i}(V) \in A[V]$. Let $\tilde{V}=\left(\tilde{V}_{1}, \ldots, \tilde{V}_{s}\right)$ be an $s$-tuple of independent variables. For each $i, 1 \leq i \leq s$, define $\tilde{z}_{i}:=\beta_{i}(\tilde{V})$. Let $y=\left\{y_{1}, \ldots, y_{a}\right\} \subset B$ be a set of generators of $P$. Let $U=\left\{U_{i j} \mid 1 \leq i \leq a, 1 \leq j \leq k\right\}, W=\left\{W_{i j} \mid 1 \leq i, j \leq a\right\}$, and $X=\left\{X_{i \alpha} \mid 1 \leq\right.$ $\left.i \leq q, \alpha \in \mathbb{N}_{0}^{a},|\alpha|=t^{\prime}\right\}$ be independent variables.

Let $\tilde{A}_{1}$ denote the $A$-algebra with generators $Y, \tilde{V}, U, W, X$, and relations

$$
\begin{array}{cc}
Q_{i}=\sum_{j=1}^{k} U_{i j} \tilde{z}_{j}-\sum_{p=1}^{a} W_{i p} Y_{p}, & 1 \leq i \leq a, \\
H_{i}=h_{i}\left(\tilde{V}_{1}, \ldots, \tilde{V}_{s}\right)+\sum_{|\alpha|=t^{\prime}} Y^{\alpha} X_{i \alpha} & 1 \leq i \leq q,
\end{array}
$$

and let $\tilde{A}:=\left(\tilde{A}_{1}\right)_{P \cap \tilde{A}_{1}}$. Define the map $\tilde{\sigma}: \tilde{A} \rightarrow B$ as follows. For each $i, 1 \leq i \leq s$, choose an element $\tilde{v}_{i} \in\left(v_{i}+P^{t^{\prime}} \hat{B}\right) \cap B$. Put $\tilde{\sigma}\left(Y_{i}\right)=y_{i}$ and $\tilde{\sigma}\left(\tilde{V}_{i}\right)=\tilde{v}_{i}$. Since $h_{i}(\tilde{v}) \in P^{t^{\prime}}, \tilde{\sigma}$ extends to a homomorphism $\tilde{\sigma}: \frac{A[Y, \tilde{V}, X]}{(H)} \rightarrow B$, which agrees with $\bar{\sigma}$ $\bmod P^{t^{\prime}}$. Finally, $\bar{m} \hat{B}=P \hat{B}$ by the choice of $\bar{A}$, hence $(\tilde{z}) \hat{B}=P \hat{B}$ by Lemma 5.6 (since $(z) \hat{B}=\bar{m} \hat{B}=P \hat{B}$ and $\left.\bar{\sigma}\left(z_{i}\right)-\tilde{\sigma}\left(\tilde{z}_{i}\right) \in P^{t^{\prime}} \hat{B}\right)$. Hence

$$
(\tilde{z}) B=P
$$

by faithful flatness of $\hat{B}$ over $B$. Put $\tilde{\sigma}\left(W_{i i}\right)=1$. By (8.10)-(8.11), $\tilde{\sigma}$ can be extended to a map $\tilde{\sigma}: \tilde{A} \rightarrow B$ (for example, we may take $\tilde{\sigma}\left(W_{i j}\right)=0$ for $i \neq j$ ). Let $\tilde{m}:=P \cap \tilde{A}$. By construction,

$$
(Y) \tilde{A} \subset(\tilde{z}) \tilde{A}
$$

(since one can solve for $Y$ in the system of linear equations $Q_{1}, \ldots, Q_{a}$ ). On the other hand, $\frac{\tilde{A}}{(Y, \tilde{z})} \cong \frac{A[V]_{P \cap A[V]}}{\left(h, \beta_{1}(V), \ldots, \beta_{k}(V)\right)} \cong \frac{\bar{A}}{\bar{m}}$ which is a field. Thus

$$
(\tilde{z}) \tilde{A}=(\tilde{z}, Y) \tilde{A}=\tilde{m} \text {. }
$$

We have

$$
(Y) \subset \Delta_{(Q, H),(W, X)} ;
$$

in particular,

$$
(Y) \subset H_{\tilde{A} / A}
$$


On the other hand, after localization at $\tilde{m}$ the equations (8.10) become smooth in view of (8.9), so that $H_{\tilde{A} / A} \not \subset \tilde{m}$. Together with (8.15) this means that

$$
H_{\tilde{A} / A} B=B \text {. }
$$

Let $C^{\prime}=\frac{\tilde{A}\left[\tilde{u}_{1}, \ldots, \tilde{u}_{\tilde{n}}\right]}{\tilde{I}}$ be an $\tilde{A}$-algebra. Consider a generalized blowing up $\pi_{z, Y^{\alpha}}$ : $C^{\prime} \rightarrow C_{1}^{\prime}$ of $C^{\prime}$ along $\tilde{m}$, with generators $\tilde{z}_{1}, \ldots, \tilde{z}_{k},\left\{Y^{\alpha}\left|\alpha \in \mathbb{N}_{0}^{a},\right| \alpha \mid=t^{\prime}\right\}$. By definition, such a blowing up is described by the equations

$$
R_{i}=\tilde{u}_{i}-\tilde{c}_{i}-\sum_{j=1}^{k} \tilde{z}_{j} \tilde{u}_{i j}^{(1)}-\sum_{|\alpha|=t^{\prime}} Y^{\alpha} X_{i \alpha}^{(1)}=0 .
$$

The key property of this transformation needed below is the fact that the $R_{i}$ are linear in $X^{(1)}$ and that

$$
(Y) \subset \Delta_{R, X^{(1)}}
$$

(immediate from definitions).

Consider the $A$-subalgebra $A\left[\tilde{V}_{1}, \ldots, \tilde{V}_{s}\right] \subset \tilde{A}$ (using (8.10), the map $\gamma$ defined in (8.19) right below and the fact that $a \neq 0$ by Remark 8.1 , it is easy to see that the $\tilde{V}$ satisfy no algebraic relations over $A$ in $\tilde{A}$ ). Define the map

$$
\begin{aligned}
\gamma: A\left[\tilde{V}_{1}, \ldots, \tilde{V}_{s}\right] & \rightarrow \bar{A} \quad \text { by } \\
\gamma\left(\tilde{V}_{i}\right) & =V_{i} .
\end{aligned}
$$

The homomorphisms $\tilde{\sigma}$ and $\bar{\sigma} \circ \gamma$ agree $\bmod P^{t^{\prime}}$. Let $\tilde{A}_{0}=\frac{A[\tilde{V}]}{\left(h(\tilde{V}),(\tilde{z})^{t^{\prime}}\right)}, \bar{A}_{0}=\frac{\bar{A}}{\bar{m}^{t^{\prime}}}$. From the equations $(8.10)$, we see that $\tilde{A}_{0}$ is a subalgebra of $\frac{\tilde{A}}{\tilde{m}^{t^{\prime}}}$ and that $\frac{\tilde{A}}{\tilde{m}^{t^{\prime}}}$ is a free $\tilde{A}_{0}$-module. We have a commutative diagram

$$
\begin{array}{ll}
\bar{A}_{0} \stackrel{\bar{\sigma} \otimes_{\bar{A}} \bar{A}_{0}}{\longrightarrow} & \frac{B}{P^{t^{\prime}}} \\
\uparrow \gamma \otimes_{\bar{A}} \bar{A}_{0} & \uparrow_{\tilde{\sigma} \otimes_{\tilde{A}} \tilde{A}_{0}} \\
\tilde{A}_{0} \longrightarrow \frac{\tilde{A}}{\tilde{m}^{t^{\prime}}}
\end{array}
$$

where $\gamma \otimes_{\bar{A}} \bar{A}_{0}$ is an isomorphism.

Definition 8.4. Let $t$ be a positive integer. Assume that we are given an $\bar{A}$-algebra $C_{1}$ and an $\tilde{A}$-algebra $\tilde{C}_{1}$, with maps $\bar{\rho}_{1}: C_{1} \rightarrow B$ and $\tilde{\rho}_{1}: \tilde{C}_{1} \rightarrow B$. We say that $\tilde{C}_{1} t$-approximates $C_{1}$ if we have a commutative diagram

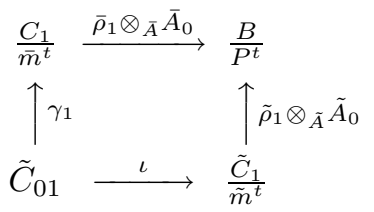

compatible with $(8.20)$, where $\tilde{C}_{01}$ is an $\tilde{A}_{0}$-algebra and $\gamma_{1}$ is an isomorphism. Suppose that $\tilde{C}_{1} t$-approximates $C_{1}$. We say that a $\tilde{C}_{1}$-module $\tilde{M} t$-approximates a $C_{1}$-module $M$ if there are $\tilde{A}_{0}$-modules $\tilde{M}_{0}$ and $\tilde{M}_{1}$ and homomorphisms $\frac{M}{\bar{m}^{t}} \stackrel{\gamma_{M}}{\longleftarrow}$ $\tilde{M}_{0} \stackrel{\iota_{M}}{\longrightarrow} \tilde{M}_{1} \cong \tilde{M}_{0} \otimes_{\tilde{C}_{01}} \frac{\tilde{C}_{1}}{\tilde{m}^{t}}$, compatible with (8.21), where $\gamma_{M}$ is an isomorphism, $\iota_{M}$ identifies $\tilde{M}_{0}$ with $\tilde{M}_{0} \otimes_{\tilde{C}_{01}} 1$, and $\tilde{M}_{1}$ is a direct summand of $\frac{\tilde{M}}{\tilde{m}^{t}}$. Suppose $\tilde{M}$ approximates $M$ and let $f \in M, \tilde{f} \in \tilde{M}$. We say that $\tilde{f} t$-approximates $f$ if the 
natural image $\tilde{f}_{0}$ of $\tilde{f}$ in $\frac{\tilde{M}}{\tilde{m}^{t}}$ lies in $\iota_{M}\left(\tilde{M}_{0}\right)$ and $\left(\gamma_{M} \circ \iota_{M}^{-1}\right)\left(\tilde{f}_{0}\right)$ is the image of $f$ in $\frac{M}{\bar{m}^{t}}$. Consider submodules $M^{\prime} \subset M, \tilde{M}^{\prime} \subset \tilde{M}$. We say that $\tilde{M}^{\prime} t$-approximates $M^{\prime}$ (as submodules of $\tilde{M}$ and $M$, respectively) if there exist sets of generators $\left(f_{1}, \ldots, f_{r}\right)$ of $M^{\prime}+\bar{m}^{t} M \bmod \bar{m}^{t} M$ and $\left(\tilde{f}_{1}, \ldots, \tilde{f}_{r}\right)$ of $\tilde{M}^{\prime}+\tilde{m}^{t} \tilde{M} \bmod \tilde{m}^{t} \tilde{M}$ such that $\tilde{f}_{j}$ $t$-approximates $f_{j}, 1 \leq j \leq r$. A homomorphism $\tilde{\phi}: \tilde{M} \rightarrow \tilde{M}^{\prime}$ of $\tilde{C}_{1}$-modules $t$-approximates a homomorphism $\phi: M \rightarrow M^{\prime}$ of $C_{1}$-modules if $\tilde{M}$-approximates $M, \tilde{M}^{\prime} t$-approximates $M^{\prime}, \tilde{\phi}_{1}\left(\tilde{M}_{1}\right) \subset \tilde{M}_{1}^{\prime}$ and $\phi$ and $\tilde{\phi}$ are compatible with the maps $\gamma_{M}, \iota_{M}, \gamma_{M^{\prime}}$ and $\iota_{M^{\prime}}$

Lemma 8.5. Let $\tilde{\phi}: \tilde{M} \rightarrow \tilde{M}^{\prime}$ be a surjective homomorphism of $\tilde{C}_{1}$-modules $t$ approximating a homomorphism $\phi: M \rightarrow M^{\prime}$ of $C_{1}$-modules. If $f \in M, \tilde{f}^{\prime} \in$ $\tilde{M}^{\prime}$ are such that $\tilde{f}^{\prime} t$-approximates $\phi(f)$, then there exists $\tilde{f} \in \tilde{\phi}^{-1}\left(\tilde{f}^{\prime}\right)$ which $t$ approximates $f$.

Proof. Straightforward diagram chasing.

Lemma 8.6. Let $\tilde{C}_{1}$ be an $\tilde{A}$-algebra essentially of finite type, $t^{\prime}$-approximating an $\bar{A}$-algebra $C_{1}$ essentially of finite type.

(1) If $\rho_{1}\left(C_{1}\right) \subset \bar{\sigma}(\bar{A})+\bar{m} B$, then $\tilde{\rho}_{1}\left(\tilde{C}_{1}\right) \subset \tilde{\sigma}(\tilde{A})+\tilde{m} B$.

(2) Let $\pi_{z}: C_{1} \rightarrow C_{2}$ be a generalized blowing up along $\bar{m}$ with generators $z_{1}, \ldots, z_{k}$, as in (4.2), and $\pi_{\tilde{z}, Y^{\alpha}}: \tilde{C}_{1} \rightarrow \tilde{C}_{2}$ a generalized blowing up along $\tilde{m}$ with generators $\tilde{z}_{1}, \ldots, \tilde{z}_{k}, Y^{\alpha},|\alpha|=t^{\prime}$, as in (8.17). Choose $\pi_{z}$ and $\pi_{\tilde{z}, Y \alpha}$ in such a way that for each $i, 1 \leq i \leq n_{1}$, the element $\tilde{c}_{i}$ of (8.17) $t^{\prime}$-approximates $c_{i}$ of (4.2) (in this case, we will say that $\pi_{\tilde{z}, Y^{\alpha}} t^{\prime}$-approximates $\pi_{z}$ ). Then $\tilde{C}_{2}$ $t^{\prime}$-approximates $C_{2}$.

(3) Let

$$
\begin{aligned}
\pi_{z}^{\prime}: \bar{A}\left[u^{(1)}\right] & \rightarrow \bar{A}\left[u^{(2)}\right] \quad \text { and } \\
\tilde{\pi}^{\prime}: \tilde{A}\left[\tilde{u}^{(1)}, X^{(1)}\right] & \rightarrow \tilde{A}\left[\tilde{u}^{(2)}, X^{(2)}\right]
\end{aligned}
$$

be the maps which induce $\pi_{z}$ and $\pi_{\tilde{z}, Y^{\alpha}}$, respectively. If an element $\tilde{f} \in$ $\tilde{A}\left[\tilde{u}^{(1)}, X^{(1)}\right] t^{\prime}$-approximates $f \in \bar{A}\left[u^{(1)}\right]$, then $\tilde{\pi}^{\prime}(\tilde{f}) t^{\prime}$-approximates $\pi_{z}^{\prime}(f)$ (in other words, the relation of $t^{\prime}$-approximation is preserved by generalized blowings up which approximate each other). The same statement is true for an element of $\tilde{C}_{1} t^{\prime}$-approximating an element of $C_{1}$, and also an element of a free $\tilde{C}_{1}$-module $\tilde{M}, t^{\prime}$-approximating an element of a free $C_{1}$-module $M, t^{\prime}$ approximated by $\tilde{M}$; ditto for a submodule of $\tilde{M} t^{\prime}$-approximating a submodule of $M$.

Proof. Immediate from definitions.

Let $L$ and $N$ be as in (8.1) and Properties 8.3. Let (8.7) be a sequence of $N+L$ generalized blowings up of $C \otimes_{A} \tilde{A}$ along $\tilde{m}$, with generators $\tilde{z}_{1}, \ldots, \tilde{z}_{k},\left\{Y^{\alpha}\right\}$. In view of (8.19), the $\tilde{A}$-algebra $C \otimes_{A} \tilde{A} t^{\prime}$-approximates the $\bar{A}$-algebra $C \otimes_{A} \bar{A}$. By Lemma 8.6 (1) and induction on $N+L$, we have that $\tilde{\rho}_{i}\left(\tilde{C}_{i}\right) \subset \tilde{\sigma}(\tilde{A})+\tilde{m} B$ for all $i<N+L$, so that such a sequence (8.7) is well defined. Moreover, by Lemma 8.6 (2) and induction on $N+L$, the sequence (8.7) $t^{\prime}$-approximates the sequence $\pi^{(N)} \circ \pi^{(L)}$ of $(8.1)$. 
Lemma 8.7. Let $t \in \mathbb{N}$. Let $C_{1}$ be an $\bar{A}$-algebra with $\bar{m} \subset J a c\left(C_{1}\right)$ and $\tilde{C}_{1}$ an $\tilde{A}$ algebra with $\tilde{m} \subset \operatorname{Jac}\left(\tilde{C}_{1}\right)$, t-approximating $C_{1}$. Let $\tilde{M}$ be a finitely generated $\tilde{C}_{1}$ module t-approximating a finitely generated $\bar{A}$-module $M$ and $\tilde{J} \subset \tilde{M}$ a submodule t-approximating a submodule $J \subset M$. Assume that $\tilde{M}_{1}=\frac{\tilde{M}}{\tilde{m}^{t}}$ (in the notation of Definition 8.4). Let $s$ be a positive integer such that

$$
s<t .
$$

If

$$
\bar{m}^{s} M \subset J,
$$

then $\tilde{m}^{s} \tilde{M} \subset \tilde{J}$.

Proof. Let $\tilde{M}_{0}$ be as in Definition 8.4. By definition of approximation, there exist generators $\tilde{f}_{1}, \ldots, \tilde{f}_{r}$ of $\frac{\tilde{J}}{\tilde{J} \cap \tilde{m}^{t} \tilde{M}_{\tilde{M}}} t$-approximating generators $f_{1}, \ldots, f_{r}$ of $\frac{J}{J \cap \tilde{m}^{t} M}$. By definition of approximation, $\tilde{f}_{j} \in \tilde{M}_{0}$. By (8.23)-(8.24), $\frac{\bar{m}^{s} M}{\bar{m}^{t} M} \subset \frac{J}{\bar{m}^{t} M}$. Applying the isomorphism $\gamma_{M}$ of Definition 8.4, we get $(\tilde{z})^{s} \tilde{M}_{0} \subset\left(\tilde{f}_{1}, \ldots, \tilde{f}_{r}\right) \tilde{M}_{0}$, so that $\frac{(\tilde{z})^{s} \tilde{M}}{(\tilde{z})^{t} \tilde{M}} \equiv \frac{\tilde{m}^{s} \tilde{M}}{\tilde{m}^{t} \tilde{M}} \subset\left(\tilde{f}_{1}, \ldots, \tilde{f}_{r}\right) \frac{\tilde{M}}{\tilde{m}^{t} \tilde{M}}$ (here we are using that $\tilde{M}_{1}=\frac{\tilde{M}}{\tilde{m}^{t}}$, so that $\frac{\tilde{M}}{\tilde{m}^{t}} \cong$ $\left.\tilde{M}_{0} \otimes_{\tilde{C}_{01}} \frac{\tilde{C}_{1}}{\tilde{m}^{t}}\right)$. By (8.23) and Nakayama's Lemma, $\tilde{m}^{s} \tilde{M} \subset \tilde{J}$, as desired.

Next, we apply the results of $\S 5$ to (8.7). By Lemma 8.6 (3) and induction on $L, \tilde{m}^{L_{0}} H_{C / A}\left(\tilde{C}_{L_{0}}\right)_{P \cap \tilde{C}_{L_{0}}} t^{\prime}$-approximates $\bar{m}^{L_{0}} H_{C / A}\left(C_{L_{0}}\right)_{P \cap C_{L_{0}}}$ (as submodules of $\left(\tilde{C}_{L_{0}}\right)_{P \cap \tilde{C}_{L_{0}}}$ and $\left(C_{L_{0}}\right)_{P \cap C_{L_{0}}}$, respectively). By the choice of $L_{0}$ and Lemma 8.7, $\tilde{m}^{L_{0}}\left(\tilde{C}_{L_{0}}\right)_{P \cap \tilde{C}_{L_{0}}} \subset \tilde{m} H_{C / A}\left(\tilde{C}_{L_{0}}\right)_{P \cap \tilde{C}_{L_{0}}}$. Thus the hypotheses of Lemma 5.4 are satisfied for $\tilde{\pi}_{L_{0}} \circ \cdots \circ \tilde{\pi}_{1}$, and so $\tilde{C}_{L}$ is an almost complete intersection at $P \cap \tilde{C}_{L}$. Pick and fix a set $w_{1}, \ldots, w_{b}$ of generators of $H_{C / A}^{L_{1}}$. Write

$$
\tilde{C}_{i}=\frac{\tilde{A}\left[\tilde{u}_{1}^{(i)}, \ldots, \tilde{u}_{n_{i}}^{(i)}, X^{(i)}\right]}{\tilde{I}_{i}}
$$

where $\tilde{u}_{j}^{(i)} t^{\prime}$-approximates $u_{j}^{(i)}, 1 \leq j \leq n_{i}$. Let $k_{i}$ be the number of the $X^{(i)}$ variables. Applying Lemma 8.6 (and induction on $L$ ) to each $w_{s} \tilde{d}_{0}\left(\bar{f}_{j}\right) \in C^{n}$, we get that its image in $\tilde{C}_{L}^{n} t^{\prime}$-approximates its image in $C_{L}^{n}$. By the choice of $L_{1}$, this implies that $\tilde{z}^{\alpha} \tilde{d}_{0}\left(\bar{f}_{j}\right) \in \tilde{C}_{L}^{n}$, where $|\alpha|=L, t^{\prime}$-approximates $z^{\alpha} \tilde{d}_{0}\left(\bar{f}_{j}\right) \in C_{L}^{n}$. Hence $\tilde{d}_{L}\left(\left(\tilde{\pi}^{(L)}\right)^{*} \bar{f}_{j}\right) \in \tilde{C}_{L}^{n_{L}+k_{L}} t^{\prime}$-approximates $d_{L}\left(\left(\pi^{(L)}\right)^{*} \bar{f}_{j}\right) \in \bar{C}_{L}^{n_{L}}$ (note that $\bmod \tilde{m}^{t^{\prime}}$ the last $k_{L}$ components of $\tilde{d}_{L}\left(\left(\tilde{\pi}^{(L)}\right)^{*} \bar{f}_{j}\right)$ in $\tilde{C}_{L}^{n_{L}+k_{L}}$ are 0$)$. Moreover, this property does not change after multiplying, respectively, by an element $y \in \bar{C}_{L} \backslash\left(P \cap \bar{C}_{L}\right)$ and $\tilde{y} \in \tilde{C}_{L} \backslash\left(P \cap \tilde{C}_{L}\right)$, $t^{\prime}$-approximating $y$. By condition (4) in the definition of $t^{\prime}, \tilde{d}_{L}\left(\left(\tilde{\pi}^{(L)}\right)^{*} \bar{f}_{j}\right)$ as an element of $\tilde{d}_{L}\left(\frac{\tilde{I}_{L}}{\tilde{I}_{L}^{2}}\right)(2 t)$-approximates $d_{L}\left(\left(\pi^{(L)}\right)^{*} \bar{f}_{j}\right)$ as an element of $d_{L}\left(\frac{I_{L}}{I_{L}^{2}}\right)$. By Lemma 8.5, applied to the $\tilde{C}_{L}$-module homomorphism $\frac{\tilde{I}_{L}}{\tilde{I}_{L}^{2}} \rightarrow \tilde{d}_{L}\left(\frac{\tilde{I}_{L}}{\tilde{I}_{L}^{2}}\right), \quad(2 t)$-approximating the $C_{L}$-module homomorphism $\frac{I_{L}}{I_{L}^{2}} \rightarrow d_{L}\left(\frac{I_{L}}{I_{L}^{2}}\right)$, the inverse image $\tilde{f}_{j}^{(L)}$ of $\tilde{d}_{L}\left(\left(\tilde{\pi}^{(L)}\right)^{*} \bar{f}_{j}\right)$ in $\frac{\tilde{I}_{L}}{\tilde{I}_{L}^{2}}$ can be chosen to $(2 t)$-approximate $\bar{f}_{j}^{(L)} \in \frac{I_{L}}{I_{L}^{2}}$. Next, apply Lemma 8.5 to the $\tilde{C}_{L}$-module homomorphism $\tilde{I}_{L} \rightarrow \frac{\tilde{I}_{L}}{\tilde{I}_{L}^{2}},(2 t)$-approximating the $C_{L}$-module homomorphism $I_{L} \rightarrow \frac{I_{L}}{I_{L}^{2}}$. By Lemma 8.5, the elements $\tilde{f}_{1}^{(L)}, \ldots, \tilde{f}_{r}^{(L)} \in \tilde{I}_{L}$ of Lemma 5.5 can be chosen 
to $(2 t)$-approximate $f_{1}^{(L)}, \ldots, f_{r}^{(L)} \in I_{L}$ of $(8.1)$. Hence $\tilde{f}_{1}^{(N+L)}, \ldots, \tilde{f}_{r}^{(N+L)}(2 t)$ approximate $f_{1}^{(N+L)}, \ldots, f_{r}^{(N+L)}$. By (8.3) and Lemma 8.7 (applied to $B$ viewed both as an $\bar{A}$-algebra and an $\tilde{A}$-algebra), $\tilde{m}^{N} B^{r} \subset J\left(\tilde{f}^{(L)}\right)$. Also, $I\left(\tilde{f}^{(L)}\right)(2 t)$ approximates $I\left(f^{(L)}\right)$. By $(8.5)$ and Lemma 8.7,

$$
\tilde{m}^{2 N} \subset I\left(\tilde{f}^{(N+L)}\right) .
$$

Thus, the hypotheses of Lemma 4.4 hold for $\tilde{C}_{L+N}$, including (4.16). Applying Lemma 4.4, we get a diagram (1.7), satisfying (1)-(5) and (7)-(8) of Proposition 1.6. It remains to prove (6) of Proposition 1.6. This is given by the following lemma.

Since $I\left(\tilde{f}^{(N+L)}\right)(2 t)$-approximates $I\left(f^{(N+L)}\right)$, they have the same minimal number of generators in view of (8.5) and Nakayama's Lemma. Let $\tilde{a}_{1}, \ldots, \tilde{a}_{l}$ be generators of $I\left(\tilde{f}^{(N+L)}\right),(2 t)$-approximating $a_{1}, \ldots, a_{l}$ of $(8.4)$. View $\tilde{f}^{(N+L)}$ as a column $r$-vector and write $\tilde{f}^{(N+L)}=\sum_{i=1}^{l} \tilde{a}_{i} \tilde{g}_{i}, g_{i} \in \tilde{A}\left[\tilde{u}^{(N+L)}, X^{(N+L)}\right]$. Let $K_{\tilde{A}}$ (resp. $K_{B}$ ) denote the kernel of the map $\tilde{A}^{l} \rightarrow \tilde{A}^{r}$ (resp. $B^{l} \rightarrow B^{r}$ ) given by the matrix $\left(\tilde{a}_{1}, \ldots, \tilde{a}_{l}\right)$. Let $K_{\bar{A}}$ (resp. $\left.K_{\hat{B}}\right)$ be the kernel of the map $\bar{A}^{l} \rightarrow \bar{A}^{r}$ (resp. $\hat{B}^{l} \rightarrow \hat{B}^{r}$ ) given by $\left(a_{1}, \ldots, a_{l}\right)$. Let $\tilde{\mathbf{g}}_{\mathbf{i}}$ denote the image of $\tilde{g}_{i}$ in $B$ and let $\tilde{\mathbf{g}}$ be the column $l$-vector with entries $\mathbf{g}_{j}$. By construction, $\tilde{\mathbf{g}} \in K_{B}$.

Lemma 8.8. We have $K_{B}=K_{\tilde{A}} B^{l}$. In particular, $\tilde{\mathbf{g}} \in K_{\tilde{A}} B^{l}$.

Proof. Of course, $K_{\tilde{A}} B^{l} \subset K_{B}$. It remains to prove the opposite inclusion. Since $\bar{\sigma}\left(a_{i}\right)-\tilde{\sigma}\left(\tilde{a}_{i}\right) \in P^{2 t} \hat{B}^{r}$ and since $P^{t} \hat{B}^{r} \subset I\left(\tilde{f}^{(N+L)}\right) B^{r}$ by (8.11), (8.13), (8.26) and the choice of $t$, there exists an $l \times l$ invertible matrix $U$ with entries in $\hat{B}$ such that

$$
\left(\sigma^{\prime}\left(a_{1}\right), \ldots, \sigma^{\prime}\left(a_{l}\right)\right) U=\left(\tilde{\sigma}\left(\tilde{a}_{1}\right), \ldots, \tilde{\sigma}\left(\tilde{a}_{l}\right)\right) .
$$

Moreover, we may take $U$ congruent to the identity matrix mod $P^{t}$. (8.27) implies that $K_{\bar{A}} \hat{B}^{l}+P^{t} \hat{B}^{l}=K_{\tilde{A}} \hat{B}^{l}+P^{t} \hat{B}^{l}$ and that $K_{B} \hat{B}^{l} \subset K_{\hat{B}}+P^{t} \hat{B}^{l}$. Since $\hat{B}$ is flat over $\bar{A}, K_{\bar{A}} \hat{B}^{l}=K_{\hat{B}}$. We obtain

$$
K_{B} \hat{B}^{l} \subset K_{\bar{A}} \hat{B}^{l}+P^{t} \hat{B}^{l}=K_{\tilde{A}} \hat{B}^{l}+P^{t} \hat{B}^{l} .
$$

Since $K_{\tilde{A}} \hat{B}^{l} \subset K_{B} \hat{B}^{l},(8.28)$ implies that

$$
K_{B} \hat{B}^{l} \subset K_{\tilde{A}} \hat{B}^{l}+\left(P^{t} \hat{B}^{l} \cap K_{B} \hat{B}^{l}\right) .
$$

Since $K_{B} \hat{B}^{l}$ and $K_{\hat{B}}$ are isomorphic as $\hat{B}$-modules (by (8.27)) and by the choice of $t$, we have $\left(P^{t} \hat{B}^{l}\right) \cap\left(K_{B} \hat{B}^{l}\right) \subset P K_{B} \hat{B}^{l}$. Together with (8.29) this implies $K_{B} \hat{B}^{l} \subset$ $K_{\tilde{A}} \hat{B}^{l}+P K_{B} \hat{B}^{l}$, so $K_{B} \hat{B}^{l}=K_{\tilde{A}} \hat{B}^{l}$ by Nakayama's lemma. Since the map $B \rightarrow \hat{B}$ is faithfully flat, this implies $K_{B}=K_{\tilde{A}} B^{l}$, as desired. This completes the proof of Lemma 8.8 and Proposition 8.2.

Remark 8.9. Let $\tilde{A}_{1}$ be as in (8.10). By definitions, the sequence (8.7) is actually defined over $\tilde{A}_{1}$. Multiplying all the $\tilde{f}_{j}^{(N+L)}$ and the $\tilde{a}_{i}$ by an element of $\tilde{A}_{1} \backslash\left(P \cap \tilde{A}_{1}\right)$ does not affect the proof or the result. Hence the diagram (1.7) of Proposition 8.2 descends to a diagram of $\tilde{A}_{1}$-algebras, satisfying (1)-(8) of Proposition 1.6. 


\section{§9. SMOOTHING OF RING HOMOMORPHISMS}

In this section we prove

Proposition 9.1. Let the notation be as in Theorem 1.5. Then there exists a commutative diagram (1.7) satisfying (1)-(8) of Proposition 1.6.

This will complete the proof of Theorem 1.5 and (1.3).

Proof of Proposition 9.1. By Proposition 8.2 and Remark 8.9, we have a diagram (1.7) of $\tilde{A}_{1}$-algebras, with $B$ replaced by $B_{P}$. In order to descend from $B_{P}$ to $B$, we need the following delocalization lemma. Let $Y=\left(Y_{1}, \ldots, Y_{a}\right), U=\left(U_{1}, \ldots, U_{s}\right)$ and $X=\left(X_{1}, \ldots, X_{p}\right)$ be sets of independent variables, $F_{j} \in C[Y, U, X]$, and $F=\left(F_{1}, \ldots, F_{q}\right)$.

Lemma 9.2. Consider a commutative diagram

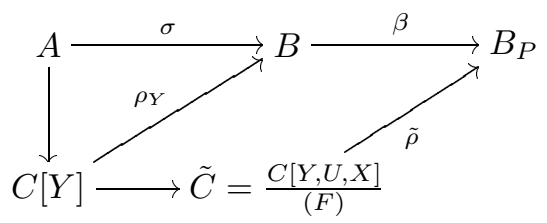

where $\left(\rho_{Y}\left(Y_{1}\right), \ldots, \rho_{Y}\left(Y_{a}\right)\right)=P$. Assume that the $F_{i}$ are linear in $X$, that the coefficients of the $X_{j}$ in $F_{i}$ do not depend on $U$ and that

$$
(Y) \tilde{C} \subset \sqrt{\Delta_{F, X} \tilde{C}}
$$

Let $E$ be an independent variable and let $U_{j}^{*}=E U_{j}$. For a positive integer $L$, put $X_{i}^{*}(L)=E^{L} X_{i}$. Let $F_{i}^{*}(L)=E^{L} F_{i}$, where we view $F_{i}^{*}(L)$ as a polynomial in $U^{*}$, $X^{*}(L)$ and $E$ over $C[Y]$. Let $C^{*}(L):=\frac{C\left[Y, U^{*}, X^{*}(L), E\right]}{\left(F^{*}(L)\right)}$; we have the obvious map $\delta: C^{*}(L) \rightarrow \tilde{C}[E]$. Then:

(1) $C^{*}(L)_{E} \cong \tilde{C}[E]_{E}$.

(2) $(Y) C^{*}(L) \subset H_{C^{*}(L) / C}$.

(3) For $L$ sufficiently large, there exists a map $\rho^{*}: C^{*}(L) \rightarrow B$, compatible with (9.1), such that $e:=\rho^{*}(E) \notin P$.

Proof. (1) is obvious. Since the relations $F$ are linear in $X$, we have $\Delta_{F, X} \equiv$ $\Delta_{F^{*}, X^{*}(L)}$ viewed as ideals in $C[Y]$. Since $(Y) \tilde{C} \subset \sqrt{\Delta_{F, X} \tilde{C}}$, we have $(Y) C^{*}(L) \subset$ $\sqrt{\Delta_{F^{*}(L), X^{*}(L)} C^{*}(L)} \subset H_{C^{*}(L) / C}$; this proves $(2)$. To prove (3), choose $e \in B \backslash P$ such that $\beta(e) \tilde{\rho}(U), \beta(e) \tilde{\rho}(X) \subset \beta(B)$ and

$$
\text { e } \operatorname{Ker} \beta=0 \text {. }
$$

For each $L \in \mathbb{N}$, pick $u_{i}^{*}, x_{j}^{*}(L) \in B, 1 \leq i \leq s, 1 \leq j \leq p$, such that $\beta\left(u_{i}^{*}\right)=$ $\beta(e) \tilde{\rho}\left(U_{i}\right)$ and $\beta\left(x_{j}^{*}(L)\right)=\beta(e)^{L} \tilde{\rho}\left(X_{j}\right)$. (9.3) implies that

$$
e x_{j}^{*}(L-1)=x_{j}^{*}(L)
$$

for all $L \geq 2$ and $1 \leq j \leq p$. Let $\gamma_{i}$ denote the degree of $F_{i}$ viewed as a polynomial in $U, 1 \leq i \leq q$. Take $L \in \mathbb{N}$ such that $L \geq 2$ and $L>\gamma_{i}$ for $1 \leq i \leq q$. Define the map $\rho^{*}: C^{*}(L) \rightarrow B$ by setting $\rho^{*}(E)=e, \rho^{*}\left(U_{i}^{*}\right)=u_{i}^{*}, \rho^{*}\left(X_{j}^{*}(L)\right)=$ $x_{j}^{*}(L)$. We have $\beta\left(F_{i}^{*}(L-1)\left(u^{*}, x^{*}(L-1)\right)\right)=\beta\left(e^{L-1}\right) F_{i}(\tilde{\rho}(U), \tilde{\rho}(X))=0$, so that $\left.F_{i}^{*}(L-1)\left(u^{*}, x^{*}(L-1)\right)\right) \in \operatorname{Ker} \beta$. Then, using $\left.(9.4), F_{i}^{*}(L)\left(u^{*}, x^{*}(L)\right)\right)=$ 
$\left.e F_{i}^{*}(L-1)\left(u^{*}, x^{*}(L-1)\right)\right) \in e \operatorname{Ker} \beta=(0)$. This shows that $\rho^{*}: C^{*}(L) \rightarrow B$ is well defined. Lemma 9.2 is proved.

Remark 9.3. If $A$ is reduced, the above argument can be modified so that $e$ is not a zero divisor in $B$. This will be important in $\S 10$ where we investigate injectivity of the map $\psi: D \rightarrow B$. Indeed, suppose $A$ is reduced. Then so is $B$. Let $Q$ be the intersection of the minimal primes of $B$ contained in $P$. Then $Q=\operatorname{Ker} \beta$. Let $P_{1}, \ldots, P_{h}$ be the minimal primes of $B$ not contained in $P$ and let $R=\bigcap_{i=1}^{h} P_{i}$ (if $h=0$, we adopt the convention $R=B)$. Then $R=A n n(\operatorname{Ker} \beta)$. In the proof of Lemma 9.2, we chose $e \in R \backslash P$. Since $Q \not \subset P_{i}$ for $1 \leq i \leq h, Q \not \subset \bigcup_{i=1}^{h} P_{i}$ for $1 \leq i \leq h$. Hence there exists $b \in Q$ such that $b \notin P_{i}$ for $1 \leq i \leq h$. Replacing $e$ by $e+b$, we may assume that $e$ is not a zero divisor and that $e^{N} \operatorname{Ker} \beta \subset Q^{N} \subset P^{N}$ for all $N \in \mathbb{N}$. Since $(Y) \subset \sqrt{\Delta_{F, X}}$, there exists $N \in \mathbb{N}$ such that

$$
P^{N} B^{q} \equiv(Y)^{N} B^{q} \subset\left(\frac{\partial F}{\partial X_{1}}, \ldots, \frac{\partial F}{\partial X_{p}}\right)
$$

(where we view the $\frac{\partial F}{\partial X_{p}}$ as $q$-vectors). Choosing $L>\gamma_{i}+N$ in the proof of Lemma 8.2, we get a map $C^{*} \rightarrow \frac{B}{P^{N}}$ compatible with (9.1). Using (9.5) and the fact that the $F_{i}$ are linear in the $X_{j}$, we lift this map to $\rho^{*}: C^{*} \rightarrow B$ as desired.

We come back to the proof of Proposition 9.1. First, note that the only requirement on the elements $y_{1}, \ldots, y_{a} \in B_{P}$ in the proof of Proposition 8.2 was that they generate $P B_{P}$. Thus, without loss of generality, we may assume that $y_{1}, \ldots, y_{a} \in B$ and that $\left(y_{1}, \ldots, y_{a}\right) B=P$. Let the notation be as in (8.10), (8.17) and (8.25). Write $\tilde{C}_{N+L}=\frac{C\left[Y, \tilde{V}, U, W, X, X^{(1)}, \ldots, X^{(N+L)}, \tilde{u}\right]}{(Q, H, R)}$. Let $\bar{X}=\left(X^{(1)}, \ldots, X^{(N+L)}\right)$. Let $(W, X, \bar{X})$ play the role of $X$ and $(U, \tilde{V})$ the role of $U$ in Lemma 9.2. (8.14) and (8.18) imply that the hypothesis (9.2) of Lemma 9.2 is satisfied. By Lemma 9.2, we obtain a $C[Y]$-algebra $C_{N+L}^{*}=\frac{C\left[Y, V^{*}, U^{*}, W^{*}, X^{*}, \bar{X}^{*}, u^{*}, E\right]}{\left(Q^{*}, H^{*}, R^{*}\right)}$ together with homomorphism $\delta: C_{N+L}^{*} \rightarrow \tilde{C}_{N+L}[E]$, which becomes an isomorphism after localization by $E$. Let $A^{*}=\frac{A\left[Y, V^{*}, U^{*}, W^{*}, X^{*}, E\right]}{\left(Q^{*}, H^{*}\right)}$. The algebra $A^{*}$ is nothing but the result of the application of Lemma 9.2 to the $A$-algebra homomorphism $A[Y] \rightarrow \frac{A[Y, \tilde{V}, U, W, X]}{(Q, H)}$. In paritcular, we have a map $\alpha: A^{*} \rightarrow \tilde{A}_{1}$ which induces an isomorphism

$$
\begin{aligned}
A_{E}^{*} & \cong \tilde{A}_{1}[E]_{E} ; \quad \text { moreover } \\
(Y) A^{*} & \subset H_{A^{*} / A} .
\end{aligned}
$$

Next, we delocalize the sequence $\tilde{C}_{0} \stackrel{\tilde{g}}{\rightarrow} \tilde{C} \stackrel{\tilde{\lambda}}{\rightarrow} \tilde{C}_{N+L}$. Namely, choose a positive integer $S$ sufficiently large so that there exist $a_{i}^{*} \in A^{* r}$ and $g_{i}^{*} \in A^{*}\left[u^{*}, \bar{X}^{*}\right]$ such that $\alpha\left(a_{i}^{*}\right)=E^{S} \tilde{a}_{i}, \delta\left(g_{i}^{*}\right)=E^{S} \tilde{g}_{i}$ and $\sum_{i=1}^{l} a_{i}^{*} g_{i}^{*}=0$ in $C_{N+L}^{*}$. Let $\mathbf{g}^{*}$ denote the column $l$-vector whose $i$ th entry is the image of $\tilde{g}_{i}$ in $B$, multiplied by $e^{S}$. Since the matrices $\left(a_{1}^{*}, \ldots, a_{l}^{*}\right)$ and $\left(\tilde{a}_{1}, \ldots, \tilde{a}_{l}\right)$ differ by a factor of the form $E^{T}, T \in \mathbb{N}_{0}$, we have $K_{A^{*}} B_{P}^{l}=K_{\tilde{A}} B_{P}^{l}$. By Lemma 8.8, $\tilde{\mathrm{g}} \in K_{\tilde{A}} B_{P}^{l}$. Hence by choosing $S$ sufficiently large, we can ensure that

$$
\mathbf{g}^{*} \in K_{A^{*}} B
$$


Put $G_{i}^{*}:=E^{S} \tilde{G}_{i}, C_{0}^{*}:=\frac{A^{*}\left[G^{*}\right]}{\left(\sum_{i=1}^{l} a_{i}^{*} G_{i}^{*}\right)}$ and $C^{*}:=\frac{A^{*}\left[u^{*}, X^{*}\right]}{\left(\sum_{i=1}^{l} a_{i}^{*} g_{i}^{*}\right)}$. We have the obvious maps : $C_{0}^{*} \stackrel{g^{*}}{\longrightarrow} C^{*} \stackrel{\lambda^{*}}{\longrightarrow} C_{N+L}^{*}$. We claim that the resulting diagram (1.7) satisfies (1)-(8) of Proposition 1.6. Indeed, (1) follows from (9.7). (2) holds because $Y_{i} \in m^{*}$, by definition of $Y$. (3) is given by (9.7), Lemma 9.2 (2), the definition of $Y$ and the transitivity and base change properties of smoothness. (5) is trivial and (6) is nothing but (9.8). Finally, (4), (7) and (8) are statements about localizations $C_{P \cap C^{*}}^{*}$ and $A_{m^{*}}^{*}$ and hence follow from Lemma $9.2(1),(9.6)$ and the corresponding properties of the diagram given by Proposition 8.2. This completes the proof of Proposition 9.1 and with it Theorems 1.5 and 1.2.

\section{§10. Smoothing in the Category of Subalgebras}

In this section we give an affirmative answer to Problem 1.3 when $A$ is reduced and $\frac{B}{Q}$ has infinite transcendence degree over $\kappa(A \cap Q)$ for any minimal prime $Q$ of $B$. We give two counterexamples. The first shows that the hypothesis of infinite transcendence degree is necessary. The second shows that the hypothesis that $A$ is reduced is necessary for the existence of (1.2) such that $\psi$ is injective and $H_{C / A} B \subset \sqrt{H_{D / C} B}$.

Theorem 10.1. Assume that one of the following holds.

(1) $\operatorname{dim} A=\operatorname{dim} B=0$.

(2) $A$ is reduced and for each minimal prime $Q$ of $B$, there exist infinitely many elements $T_{1}, \ldots, T_{n}, \ldots$ in $\frac{B}{Q}$, algebraically independent over $\kappa(A \cap Q)$.

Consider a diagram (1.1) with $\rho$ injective. Then there exists a diagram (1.2) such that $\psi$ is injective and $H_{C / A} B \subset \sqrt{H_{D / C} B}$.

Remark 10.2. There are two special cases in which the hypotheses of (2) are satisfied. One is when $B=A\left[\left[x_{1}, \ldots, x_{n}\right]\right]$, with $x_{i}$ independent variables, the other when $A$ is essentially of finite type over a field $k$ or $\mathbb{Z}$ and $B$ is the completion with respect to a non-zero ideal $I \subset A$. To see this in the first case, let $A_{0}$ be the subring of $A$ generated by 1 . Then $A_{0}\left[\left[x_{1}, \ldots, x_{n}\right]\right]$ has cardinality continuum, hence has uncountable transcendence degree over $A_{0}$. Since $A_{0}\left[\left[x_{1}, \ldots, x_{n}\right]\right] \otimes_{A_{0}} A \subset B, B$ has uncountable transcendence degree over $A$. The proof in the second case is similar, except we let $A_{0}$ be an essentially finite type algebra over a countable subfield $k_{0}$ of $k$, satisfying $A=A_{0} \otimes_{k_{0}} k$. This raises the question, suggested by $\mathrm{B}$. Teissier, of describing $B$ explicitly as a direct limit of its smooth subalgebras of finite type.

Proof of Theorem 10.1. Case (1). Since both $A$ and $B$ can be written as direct products of local artinian rings, it is sufficient to consider the case when $B$ is local. Then $B$ is a complete local ring and Theorem 10.1 follows from Theorem 7.10, since, in this case, the algebra $A^{*}$ of Theorem 7.10 is isomorphic to $B$ and is a filtered inductive limit of smooth local $A$-subalgebras of $B$, essentially of finite type over a polynomial ring over $A$.

Case (2). Let the notation be as in Theorem 1.5. We wish to prove that if $\rho$ is injective, we may choose $D$ to be a subalgebra of $B$ in Theorem 1.5. The idea is that in $\S \S 9-10 D$ is obtained from $C$ by a composition of two kinds of operations: adjoining algebraically independent elements and taking a finitely generated algebra defined by linear (not necessarily homogeneous) equations. Since $A$ is reduced, so are $B$ and $C$. Let $P_{1}, \ldots, P_{k}$ denote the minimal primes of $B$. Let $Q_{i}:=P_{i} \cap C$. Renumbering the $P_{i}$, we may assume that $Q_{1}, \ldots, Q_{h}$ are the minimal elements 
among the $Q_{1}, \ldots, Q_{k}, h \leq k$. Then $(0)=\bigcap_{i=1}^{h} Q_{i}$, so that $Q_{1}, \ldots, Q_{h}$ are precisely the minimal primes of $C$. Let $K$ denote the total ring of fractions of $C ; K$ is a direct product of fields.

First, suppose $P$ is a minimal prime of $B$. Since $C$ is reduced, it is non-singular at its generic points. In this case, $P \cap A$ is a minimal prime of $A$, and $\kappa(P)$ is smooth (i.e. separable) over $\kappa(P \cap A)$. Hence $\kappa(P \cap C)$ is also separable over $\kappa(P \cap A)$. If $P \cap C$ were a minimal prime of $C$, this would mean that $C$ is smooth over $A$ at $P \cap C$, which contradicts the fact that $H_{C / A} \subset P \cap C$. Thus $P \cap C$ is not a minimal prime of $C$. In the above notation, we may take $P=P_{k}$ and $h<k$. Pick an element $t \in \bigcap_{i=1}^{k-1} P_{i}$ such that $t$ is algebraically independent over $C \bmod P$. Let $x_{1}, \ldots, x_{l}$ be a set of generators of the ideal $P \cap C$. Let $T$ be an independent variable and let $D:=\frac{C[T]}{\left(x_{1} T, \ldots, x_{l} T\right)}$. Define $\psi: D \rightarrow B$ by $\psi(T)=t$. The algebraic independence of $t$ $\bmod P$ implies that $\psi$ is injective. We have $\sqrt{H_{C / A} D} \subset(P \cap C) D=H_{D / C}$. Since $D_{P \cap D} \cong \frac{C_{P \cap C}}{(x) C_{P \cap C}}(T)=\frac{C_{P \cap C}}{(P \cap C) C_{P \cap C}}(T)=\kappa(P \cap C)(T)$, and since $\kappa(P \cap C) \subset \kappa(P)$ are separable over $\kappa(P \cap A)$, we have $H_{D / A} \not \subset P \cap D$. Replace $C$ by $D$. Iterating this procedure finitely many times, we may assume that $H_{C / A}$ is not contained in any minimal prime of $B$ (hence also in no minimal prime of $C$ ).

Lemma 10.3. Under the assumptions of Theorem 10.1 (2), consider a commutative diagram

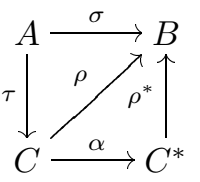

where $C^{*}=\frac{C\left[U_{1}, \ldots, U_{s}\right]}{\left(F_{1}, \ldots, F_{q}\right)}$ and the $F_{i}$ are linear in $U_{1}, \ldots, U_{s}$. Let $P$ be a non-minimal prime ideal of $B$, such that $P \cap C$ is not a minimal prime of $C$, and let $t \in \mathbb{N}$. Assume that $\rho$ is injective. Then there exists an ideal $J$, contained in the $C$-torsion submodule of $C^{*}$, and an injective homomorphism $\rho_{1}: \frac{C^{*}}{J} \rightarrow B$ such that, letting $\pi$ denote the natural map $C^{*} \rightarrow \frac{C^{*}}{J}$, we have

(1) $\rho=\rho_{1} \circ \pi \circ \alpha$.

(2) $\rho^{*}\left(U_{i}\right)-\rho_{1}\left(U_{i}\right) \in P^{t}$ for $1 \leq i \leq s$.

Furthermore, $H_{C^{*} / C} \subset \sqrt{\operatorname{Ann}_{C^{*}} J}$.

Proof. Write $F_{i}=\sum_{j=1}^{s} b_{i j} U_{j}-b_{i 0}$. Consider the linear map $b: K^{s} \rightarrow K^{q}$ given by the $q \times s$ matrix $b_{i j}, j \neq 0$. For $1 \leq j \leq h$, let $K_{j}=\kappa\left(Q_{j}\right)$. Let $r_{j}=$ $\operatorname{rk}\left(b_{i l}\right)_{\substack{1 \leq i \leq q \\ 1 \leq l \leq s}} \bmod Q_{j}$. Let $\rho_{j}^{*}$ denote the map $C^{*} \otimes_{C} K_{j} \rightarrow B \otimes_{C} K_{j}$ induced by $\rho^{*}$. Let $b_{B}$ denote the map $K^{s} \otimes_{C} B \rightarrow K^{q} \otimes_{C} B$ induced by $b$. We will show that there exists $v=\left(v_{1}, \ldots, v_{s}\right) \in\left(\operatorname{Ker} b_{B}\right) \cap P^{t} B^{s}$ such that

$$
\text { tr. } \operatorname{deg}\left(K_{j}\left[\rho_{j}^{*}\left(U_{1}\right)+v_{1}, \ldots, \rho_{j}^{*}\left(U_{s}\right)+v_{s}\right] / K_{j}\right)=s-r_{j}
$$

for $1 \leq j \leq h$. We construct $\left(v_{1}, \ldots, v_{s}\right)$ as follows. By definition of $r_{j}$, there exists a subset $S_{j} \subset\{1, \ldots, s\}$, of cardinality $s-r_{j}$, such that, fixing $s-r_{j}$ arbitrary elements $v_{i}^{(j)} \in B, i \in S_{j}$, the system of equations $\sum_{m=1}^{s} b_{m} v_{m}^{(j)}=0$ in the unknowns 
$v_{m}^{(j)}, m \notin S_{j}$ has a unique solution in $B \otimes_{C} K_{j}$. Choose $v_{i}^{(j)} \in B, i \in S_{j}$, such that the extension of $K_{j}$ which they generate in $B \otimes_{C} K_{j}$ has transcendence degree $s-r_{j}$ over $\rho_{j}^{*}\left(C^{*} \otimes K_{j}\right)$. For $i \notin S_{j}$, let $v_{i}^{(j)}$ be defined by $\sum_{i=1}^{s} b_{i} v_{i}^{(j)}=0$. Let $v^{(j)}$ denote the $s$-vector whose $i$ th entry is $v_{i}^{(j)}$. Multiplying $v^{(j)}$ by a suitable element of $C$, we may assume that $v_{i}^{(j)} \in B$ for all $i, 1 \leq i \leq s$. For each $j, 1 \leq j \leq h$, pick an element $x_{j} \in\left(P^{t} \cap C\right) \cap\left(\bigcap_{\substack{1 \leq i \leq h \\ i \neq j}} Q_{i} \backslash Q_{j}\right)$. Define $v=\sum_{j=1}^{h} x_{j} v^{(j)} \in \operatorname{Ker} b_{B} \cap P^{t} B^{s}$. Define $\rho_{1}: C^{*} \rightarrow B$ by $\rho_{1}\left(U_{i}\right)=\rho^{*}\left(U_{i}\right)+v_{i}$. For each $1 \leq j \leq h$, the map $\rho_{1 j}: C^{*} \otimes_{C} K_{j} \rightarrow B \otimes_{C} K_{j}$, induced by $\rho_{1}$, is injective. Let $J=\operatorname{Ker} \rho_{1}$. Then $A n n_{C} J$ is not contained in any minimal prime of $C$, i.e. $J$ is contained in the $C$-torsion submodule of $C^{*}$. Take a prime ideal $Q \subset C^{*}$ such that $H_{C^{*} / C} \not \subset Q$. Then $C_{Q}^{*}$ is smooth over $C$, hence has no $C$-torsion. Therefore $J$ is annihilated by some element of $C^{*} \backslash Q$, i.e. $A n n_{C^{*}} J \not \subset Q$. This proves that $H_{C^{*} / C} \subset \sqrt{A n n_{C^{*}} J}$. Lemma 10.3 is proved.

We go through the proof of Theorem 1.2 step by step and study the injectivity of all the maps to $B$ which appear along the way. Let $C=\frac{A\left[u_{1}, \ldots, u_{n}\right]}{I}$ be a presentation of $C$. Consider a diagram (1.1). The first step is to replace $C$ by the symmetric algebra $S=S_{C}\left(\frac{I}{I^{2}}\right)$ (Lemma 5.2). By Lemma 10.3 there exists an ideal $J$ contained in the $C$-torsion submodule of $S$ and an injective map $\frac{S}{J} \rightarrow B$ compatible with $\rho$. Let $\tilde{S}=\frac{S}{J}$. By Elkik's Lemma (Lemma 5.2), $S$ has a presentation such that the conormal bundle is globally trivial above the smooth locus of $S$. Since

$$
H_{C / A} S \subset H_{S / C} \subset \sqrt{A n n_{S} J},
$$

$S$ and $\tilde{S}$ are isomorphic above the smooth locus of $S$ over $C$ and $H_{C / A} \tilde{S} \subset H_{\tilde{S} / C}$. Then $H_{C / A} \tilde{S} \subset H_{\tilde{S} / A}$ by transitivity of smoothness. If $H_{\tilde{S} / A} B \not \subset P$, we may take $D=\tilde{S}$ and there is nothing more to do. Assume that $H_{\tilde{S} / A} B \subset P$; then $P$ is a minimal prime of $H_{\tilde{S} / A} B$. Hence we may replace $S$ by $\tilde{S}$ in all the subsequent arguments (note: we are not claiming that the conormal bundle of $\tilde{S}$ is free over the smooth locus of $\tilde{S}$; however, by (10.1), it is free away from the locus defined by the ideal $H:=H_{C / A} \tilde{S}$, which has the property that $\sqrt{H B_{P}}=P$ - this is what we actually used in the proof of Theorem 1.2). From now on assume that $\frac{I}{I^{2}}$ is globally free away from the locus defined by an ideal $H$, such that $\sqrt{H B_{P}}=P$. By Propositions 9.1 and 1.6 (and by the proof of Proposition 9.1), there exists a commutative diagram

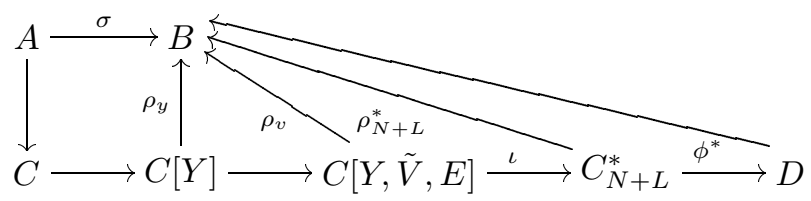

such that $C[Y, \tilde{V}, E]$ is pure transcendental over $C$ (in particular, $C[Y, \tilde{V}, E]$ is defined by zero linear equations over $C[Y]), C_{N+L}^{*}$ is defined by linear equations 
over $C[Y, \tilde{V}, E], D$ by linear equations over $C_{N+L}^{*}$,

$$
\begin{aligned}
& (Y) \subset H_{C_{N+L}^{*} / C[Y, \tilde{V}, E]}, \\
& (Y) \subset H_{D / C_{N+L}^{*}} \text { and } \\
& H_{D / A} B \not \subset P .
\end{aligned}
$$

We want to show that $D$ can be chosen so that the map $D \rightarrow B$ is injective. First, we show that the map $\rho_{y}: C[Y] \rightarrow B$ can be chosen to be injective. In other words, we have to show that there exists a set $\left(y_{1}, \ldots, y_{a}\right)$ of generators of $P$ algebraically independent over $C$. Take a set $\left(y_{1}, \ldots, y_{a}\right)$ of generators of $P$. Suppose tr. $\operatorname{deg}(C[y]) / C)=b<a$. Say, $y_{1}, \ldots, y_{b}$ are algebraically independent over $C$ and $y_{b+1}, \ldots, y_{a}$ are algebraic over $C\left[y_{1}, \ldots, y_{b}\right]$. For each $i, 1 \leq i \leq h$, take an element $x_{i} \in B$, transcendental over $C[y] \bmod P_{i}$. It is easy to construct a $C[y]$ linear combination $x$ of the $x_{i}$, which is transcendental over $C[y] \bmod P_{i}$ for each $i, 1 \leq i \leq h$. If $b=0$, pick $w \in\left(P \backslash\left(\bigcup_{i=1}^{h} P_{i}\right)\right) \cap \rho(C)$ and define $y_{a+1}:=w x$; then $y_{a+1} \in P$ is transcendental over $C$. Next, assume $b \geq 1$. Let $y_{b+1}^{\prime}:=y_{b+1}+x y_{1}$. Replacing $y_{b+1}$ by $y_{b+1}^{\prime}$, we increase tr. $\operatorname{deg}(C[y] / C)$ by 1 . By induction on $b$, there exists a choice of generators $\left(y_{1}, \ldots, y_{a}\right)$ which are algebraically independent over $C$. That is, we may assume that $\rho_{y}$ is injective. Applying Lemma 10.2 successively to the homomorphisms $C[Y] \rightarrow C[Y, \tilde{V}, E], \rho_{v}(C[Y, \tilde{V}, E]) \rightarrow \frac{C_{N+L}^{*}}{\left(\operatorname{Ker} \rho_{v}\right) C_{N+L}^{*}}$ and $\rho_{N+L}^{*}\left(C_{N+L}^{*}\right) \rightarrow \frac{D}{\left(\operatorname{Ker} \rho_{N+L}^{*}\right) D}$, we get a commutative diagram

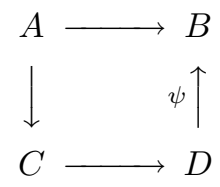

Let $J=\operatorname{Ker} \psi$. Using (10.3)-(10.4), the fact that smoothness is preserved by base change and the last statement of Lemma 10.3, we have

$$
\begin{aligned}
& (Y) D \subset H_{D / C} \quad \text { and } \\
& (Y) D \subset \sqrt{A n n_{D} J} .
\end{aligned}
$$

Let $D_{1}:=\frac{D}{J}$. By $(10.6)$ and (10.7),

$$
(Y) D_{1} \subset H_{D_{1} / C}
$$

Let $R_{1}, \ldots, R_{l}$ denote the minimal primes of $A$, contained in $P$. Then $R_{1} A_{m}, \ldots$, $R_{l} A_{m}$ are precisely the minimal primes of $A_{m}$. By regularity of $\sigma, R_{1} B_{P}, \ldots, R_{l} B_{P}$ are precisely the minimal primes of $B_{P}$. Similarly, since $D_{P \cap D}$ is smooth over $A$, $R_{1} D_{P \cap D}, \ldots, R_{l} D_{P \cap D}$ are the minimal primes of $D_{P \cap D}$. Since $(y) B=P$ is not a minimal prime of $B, \rho_{y}(Y) \not \subset R_{i} B_{P}$ for any $i$. Then $(Y) D_{P \cap D} \not \subset R_{i} D_{P \cap D}$ for any $i, 1 \leq i \leq l$. Since $(Y) D_{P \cap D}$ is not contained in any minimal prime of $D_{P \cap D}$, (10.7) implies that $J D_{P \cap D}=0$. Hence $D_{P \cap D}=\left(D_{1}\right)_{P \cap D_{1}}$ so $H_{D_{1} / A} B \not \subset P$ by (10.5) and Lemma $10.3(2)$. We have constructed a $C$-algebra $D_{1}$ together with an injective map $\psi_{1}: D_{1} \rightarrow B$, such that $\sqrt{H_{C / A} B} \subset H_{D_{1} / C} B$ (cf. (10.8)) and $H_{D_{1} / A} B \not \subset P$ (hence $\sqrt{H_{C / A} B} \varsubsetneqq H_{D_{1} / A} B$ ), as desired.

The next example shows that Theorem 10.1 may fail if $A$ is not reduced. 
Example 10.4. Let $k$ be a field and let $A:=\frac{k[x, y, z]_{(x, y, z)}}{\left(x^{2}, x y, x z\right)}$. Let $B:=A[[T]]$. Let $C:=\frac{A[u]}{(x u)}$ and define the map $\rho: C \rightarrow B$ by $\rho(u)=y T$. We want to show that there does not exist a commutative diagram (1.2) such that $\psi$ is injective and $(x, y, z) B \subset \sqrt{H_{D / C} B}$. Suppose such a diagram (1.2) exists. Since $x u=$ 0 in $D$ and since $A n n_{D} x=\left(A n n_{A} x\right) D$ by flatness, we have $\phi(u) \in(x, y, z) D$. Write $\phi(u)=x v_{1}+y v_{2}+z^{n} v_{3}$ in $D$, where either $v_{3} \notin(x, y, z) D$ or $v_{3}=0$. We have $\psi\left(x v_{1}\right) \in(y, z) B \cap(x) B=((y, z) \cap(x)) B=(0)$, so $x v_{1}=0$ in $D$ by the injectivity of $\psi$. Hence $\phi(u)=y v_{2}+z^{n} v_{3}$. We claim that $v_{3}=0$. Indeed, we have $y T=y \psi\left(v_{2}\right)+z^{n} \psi\left(v_{3}\right)$. Hence $z^{n} \psi\left(v_{3}\right) \in(y) B \cap\left(z^{n}\right) B=\left(y z^{n}\right) B$, so that $\psi\left(v_{3}\right) \in(x, y) B$. Then $\psi\left(x v_{3}\right)=x \psi\left(v_{3}\right)=0$, so $x v_{3}=0$ and hence $v_{3} \in(x, y, z) D$, which implies $v_{3}=0$. We have shown that $\phi(u) \in(y) D$. Therefore for any prime $P \subset D$ with $H_{D / C} \not \subset P$, we have $u \in(y) C_{P \cap C}$, which implies that $A n n_{C} \frac{(u, y)}{(y)} \not \subset P \cap C$. This means that $H_{D / C} \subset \sqrt{\left(A n n_{C} \frac{(u, y)}{(y)}\right) D}=\sqrt{(x, y) D}$, so that $\sqrt{H_{D / C} B} \subset(x, y) B$, which contradicts $(x, y, z) B \subset \sqrt{H_{D / C} B}$.

The next example shows that if $B$ does not have infinite transcendence degree over $A$, then Theorem 10.1 may fail, even when $A$ is regular. First, consider the analogous question for modules in Lazard's theorem. By an argument similar to that of Lemma 10.3, one shows that for a reduced $\operatorname{ring} A$, a flat module $M$ which has infinite rank over the residue field at every minimal prime of $A$, is an inductive limit of free finitely generated $A$-submodules. We give an example showing that this is not so without the infinite rank assumption. We then use it to give an example of a regular homomorphism of noetherian rings $\sigma: A \rightarrow B$ such that $B$ is not a direct limit of smooth $A$-subalgebras of finite type.

Example 10.5. Let $k_{0}$ be a field and $a_{i j}, b_{i j}, c_{i j}, j \in \mathbb{N}, 1 \leq i \leq 3$, independent variables. Let $k$ be the pure transcendental extension of $k_{0}$ with the generators $a_{i j}, b_{i j}, c_{i j}, j \in \mathbb{N}, 1 \leq i \leq 3$. Let $A:=k[x, y, z]_{(x, y, z)}$, with $x, y z$ independent variables. We define the following inductive system:

$$
M_{1} \stackrel{\phi_{1}}{\longrightarrow} M_{2} \stackrel{\phi_{2}}{\longrightarrow} \ldots \stackrel{\phi_{n-1}}{\longrightarrow} M_{n} \stackrel{\phi_{n}}{\longrightarrow} \ldots
$$

of free finitely generated $A$-modules. Put $M_{i}=A^{3}$ for all $i \in \mathbb{N}$. Let $f_{i}, g_{i}, h_{i}$ denote the generators of $M_{i}$. Let

$$
l_{i j}:=a_{i j} x+b_{i j} y+c_{i j} z,
$$

$1 \leq i \leq 3, j \in \mathbb{N}$. Define the homomorphism $\phi_{i}$ by $\phi_{i}\left(f_{i}\right)=l_{1 i} f_{i+1}+l_{2 i} g_{i+1}$, $\phi_{i}\left(g_{i}\right)=-l_{3 i} f_{i+1}+l_{2 i} h_{i+1}, \phi_{i}\left(h_{i}\right)=-l_{3 i} g_{i+1}-l_{1 i} h_{i+1}$ for $i \in \mathbb{N}$. Let $M:=\lim _{i \rightarrow \infty} M_{i}$. $M$ is an inductive limit of free $A$-modules, hence flat. We want to show that $M$ is not an inductive limit of free finitely generated $A$-submodules. Suppose the contrary. Since rk $M=2$, any free submodule of $M$ can have rank at most 2 . Hence there exists a constant $n \in \mathbb{N}$ such that, in the notation of (10.9), $M$ is also the limit of the inductive system

$$
M_{1} \stackrel{\psi}{\rightarrow} A^{2} \stackrel{\lambda}{\rightarrow} M_{n+1} \rightarrow A^{2} \rightarrow M_{2 n+1} \rightarrow \cdots .
$$

Represent $\psi$ and $\lambda$, respectively, by a $2 \times 3$ and a $3 \times 2$ matrix with entries in $A$; denote these matrices by $\Psi$ and $\Lambda$. Similarly, represent each $\phi_{i}$ by a $3 \times 3$ matrix $\Phi_{i}$ with entries in $A$. By construction, $\Lambda \Psi=\Phi_{n} \Phi_{n-1} \cdots \Phi_{1}$. Consider the $2 \times 2$ submatrix $\Phi$ of $\left(\Phi_{n} \Phi_{n-1} \cdots \Phi_{1}\right)$, obtained by deleting the third row and the third 
column of $\left(\Phi_{n} \Phi_{n-1} \cdots \Phi_{1}\right)$. To get a contradiction, it remains to prove that $\Phi$ is not a product of two non-invertible $2 \times 2$ matrices with entries in $A$ (we may assume that the first matrix is not invertible because $\operatorname{Im}(\Lambda \Psi)$ is not contained in $\Lambda \Psi\left(A f_{1}+A g_{1}\right)$, and the second because $\operatorname{Im}(\Lambda \Psi)$ is not contained in a submodule of the form $\left.A\left(f_{2}+b h_{2}\right)+A\left(g_{2}+c h_{2}\right)\right)$. It is sufficient to prove that det $\Phi$ is an irreducible polynomial in $k[x, y, z]$. The polynomial det $\Phi$ is homogeneous in $x, y$ and $z$ of degree $2 n$. Moreover, the coefficients of det $\Phi$ are homogeneous polynomials in $a_{i j}, b_{i j}$ and $c_{i j}$ of degree $2 n$, quadratic in the variables $a_{i j}, b_{i j}, c_{i j}$ for each fixed $j$. For each $i \in \mathbb{N}$, let $k_{i}$ denote the pure transcendental extension of $k_{0}$ generated by $x, y, z, a_{l j}, b_{l j}, c_{l j}$, where $j \in \mathbb{N}, j \neq i$ and $1 \leq l \leq 3$.

Lemma 10.6. The polynomial det $\Phi$, viewed as a polynomial over $k_{i}$, is an irreducible quadratic form in the nine variables $a_{j i}, b_{j i}, c_{j i}, 1 \leq j \leq 3$.

Proof. The polynomial det $\Phi$ is a quadratic form over $k_{i}$ in $a_{j i}, b_{j i}, c_{j i}, 1 \leq j \leq 3$. Let $\bar{\Phi}_{1}$ denote the $3 \times 2$ matrix given by the first two columns of $\Phi_{1}$ and $\bar{\Phi}_{n}$ the $2 \times 3$ matrix given by the first two rows of $\Phi_{n}$.

Lemma 10.7. Let $2<i<n-1$. Write $\bar{\Phi}_{n} \Phi_{n-1} \cdots \Phi_{i+1}=\left(\begin{array}{ccc}\phi_{11} & \phi_{12} & \phi_{13} \\ \phi_{21} & \phi_{22} & \phi_{23}\end{array}\right)$, $\Phi_{i-1} \cdots \Phi_{2} \bar{\Phi}_{1}=\left(\begin{array}{cc}\psi_{11} & \psi_{12} \\ \psi_{21} & \psi_{22} \\ \psi_{31} & \psi_{32}\end{array}\right)$. Then $\phi_{l j}, \psi_{l j}, a_{l i}, b_{l i}, c_{l i}, x, y, z$ are all algebraically independent over $k_{0}$.

Proof. Since each matrix $\Phi_{t}$ involves a different set of variables $a_{l t}, b_{l t}, c_{l t}$, it is sufficient to prove that each of the sets $\left\{\phi_{l j}\right\}$ and $\left\{\psi_{l j}\right\}$ separately is algebraically independent over $k_{0}(x, y, z)$. This is proved by straightforward induction on $n-i$ and $i$, respectively.

We have

$$
\Phi=\left(\begin{array}{lll}
\phi_{11} & \phi_{12} & \phi_{13} \\
\phi_{21} & \phi_{22} & \phi_{23}
\end{array}\right)\left(\begin{array}{ccc}
l_{1 i} & -l_{3 i} & 0 \\
l_{2 i} & 0 & -l_{3 i} \\
0 & l_{2 i} & -l_{1 i}
\end{array}\right)\left(\begin{array}{ll}
\psi_{11} & \psi_{12} \\
\psi_{21} & \psi_{22} \\
\psi_{31} & \psi_{32}
\end{array}\right)
$$

Lemma 10.6 follows by a direct calculation.

By Lemma 10.6, if $\operatorname{det} \Phi=f g$ is a factorization of $\operatorname{det} \Phi$, then for each $i, 1 \leq i \leq$ $n$, either $f$ or $g$ is independent of $a_{l i}, b_{l i}, c_{l i}$. In other words, there is a partition $\{1, \ldots, n\}=\Lambda_{f} \coprod \Lambda_{g}$, such that $f$ depends only on $x, y, z$ and $a_{l i}, b_{l i}, c_{l i}$ for $i \in \Lambda_{f}$ (and is a quadratic form in these nine variables for each fixed $i$ ) and similarly for $g$. Say, $1 \in \Lambda_{f}$. Let $\tilde{\Phi}:=\bar{\Phi}_{n} \Phi_{n-1} \cdots \Phi_{2}$, and write $\tilde{\Phi}=\left(\begin{array}{ccc}\alpha & \beta & \gamma \\ \delta & \epsilon & \chi\end{array}\right)$. By definition, $\bar{\Phi}_{1}=\left(\begin{array}{cc}l_{11} & -l_{31} \\ l_{21} & 0 \\ 0 & l_{21}\end{array}\right)$. Hence $\Phi=\left(\begin{array}{cc}\alpha l_{11}+\beta l_{21} & -\alpha l_{31}+\gamma l_{21} \\ \epsilon l_{21}+\delta l_{11} & -\delta l_{31}+\chi l_{21}\end{array}\right)$. Viewing det $\Phi$ as a polynomial in $a_{i 1}, b_{i 1}$ and $c_{i 1}$ over $k_{1}[x, y, z]$, the coefficient of $a_{21} a_{31}$ is $x^{2}(\alpha \epsilon-\beta \delta)$. By induction on $n, \alpha \epsilon-\beta \delta$ is an irreducible polynomial in $k[x, y, z]$. By (10.12) and Lemma 10.7, $\operatorname{det} \Phi$ is not divisible by $x$, hence $g$ is not divisible by $x$. Thus $g=\alpha \epsilon-\beta \delta$ and $f$ depends only on $a_{i 1}, b_{i 1}, c_{i 1}$ and $x, y, z$. By symmetry we can make the same argument for $n$ instead of 1 . This proves that $g$ depends only on $a_{i n}, b_{i n}, c_{i n}$ and $x, y, z$. Then $n=2$, in which case $\operatorname{det} \Phi$ is clearly irreducible. This proves that $M$ is not a direct limit of its free finitely generated submodules. 
Remark 10.8. In Example 10.5, rk $M=2$. Since $A$ is a UFD, any flat rank 1 $A$-module is a direct limit of free finitely generated submodules. However, there exists a normal local ring $A$ which is not a UFD, and an $A$-module $M$ of rank 1, which is not a direct limit of free $A$-submodules. Namely, let $k_{0}$ be a field. Let $x, y, z$ be independent variables. Consider an infinite number of variables $a_{j}, b_{j}$ and $c_{j}, j \in \mathbb{N}$. For each $j$, let $r_{j}$ and $s_{j}$ denote the roots of the quadratic equation $\left(a_{j} X+b_{j}\right)^{2}-c_{j}^{2} X=0$. Let $k$ denote the extension of $k_{0}$ generated by $a_{j}, b_{j}, c_{j}$, $r_{j}, s_{j}, j \in \mathbb{N}$. Let $A:=\frac{k[x, y, z]_{(x, y, z)}}{\left(z^{2}-x y\right)}$. We define the following inductive system:

$$
M_{1} \stackrel{\phi_{1}}{\longrightarrow} M_{2} \stackrel{\phi_{2}}{\longrightarrow} \cdots \stackrel{\phi_{n-1}}{\longrightarrow} M_{n} \stackrel{\phi_{n}}{\longrightarrow} \cdots
$$

of free finitely generated $A$-modules. Put $M_{i}:=A^{2}$ for all $i \in \mathbb{N}$. Let $f_{i}, g_{i}$ denote the generators of $M_{i}$. Let $l_{1 j}=a_{j} x+b_{j} y+c_{j} z, l_{2 j}=a_{j}\left(x-r_{j} y\right), l_{3 j}=a_{j}\left(x-s_{j} y\right)$, $l_{4 j}:=a_{j} x+b_{j} y-c_{j} z$. Define $\phi_{i}$ by $\phi_{i}\left(f_{i}\right)=l_{1 i} f_{i+1}+l_{2 i} g_{i+1}, \phi_{i}\left(g_{i}\right)=l_{3 i} f_{i+1}+l_{4 i} g_{i+1}$ for $i \in \mathbb{N}$. Let $M:=\lim _{i \rightarrow \infty} M_{i}$. The module $M$ is an inductive limit of free modules, hence flat. Since $l_{1 i} l_{4 i}=l_{2 i} l_{3 i}$ by construction, we have rk $M=1$. To prove that $M$ is not a direct limit of free submodules, represent the homomorphisms $\phi_{i}$ by $2 \times 2$ matrices $\Phi_{i}$. If $M$ is a direct limit of free submodules, these submodules must necessarily be of rank 1 . Then for some $n$ we can write $\Phi_{n} \cdots \Phi_{1}=\Lambda \Psi$, where $\Lambda$ and $\Psi$ are a column 2 -vector and a row 2 -vector, respectively. Let $\phi$ denote the left uppermost entry of the matrix $\Phi_{n} \cdots \Phi_{1}$. It is sufficient to prove that $\phi$ cannot be written as a product of two elements of $A$ in a non-trivial way. Suppose $\phi=f g$. Since, for each $i \in\{1, \ldots, n\}, \phi$ is a linear homogeneous polynomial in $a_{j}, b_{j}, c_{j}$, $r_{j}, s_{j}$, there exists a partition $\{1, \ldots, n\}=\Lambda_{f} \amalg \Lambda_{g}$ such that $f$ depends only on $x, y, z$ and $a_{j}, b_{j}, c_{j}, r_{j}, s_{j}$ for $j \in \Lambda_{f}$ and similarly for $g$. Then one uses induction on $n$ to show that $\# \Lambda_{f}, \# \Lambda_{g} \leq 1$, which easily leads to a contradiction.

Next we show how Example 10.5 and the example of Remark 10.8 can be used to construct regular homomorphisms $\sigma: A \rightarrow B$ such that $B$ is not a direct limit of smooth $A$-subalgebras of finite type. In the first example we will have tr. $\operatorname{deg}_{A} B=$ 2 , in the second tr. $\operatorname{deg}_{A} B=1$.

Example 10.9. Let $k_{0}$ be a field and let $k=k_{0}(a, b, c, d)$, where $a=\left\{a_{i j}\right\} \underset{\substack{j \in \mathbb{N} \\ 1 \leq i \leq 3}}{ }$ and similarly for $b, c$ and $d$. Let $A=k[x, y, z]_{(x, y, z)}$. Let $l_{i j}$ be as in (10.10) and let $B$ denote the ring generated over $A$ by the symbols $\left\{f_{j}, g_{j}, h_{j}\right\}_{j \in \mathbb{N}}$ with the relations

$$
\begin{aligned}
f_{i}-d_{1 i} & =l_{1 i} f_{i+1}+l_{2 i} g_{i+1}, \\
g_{i}-d_{2 i} & =-l_{3 i} f_{i+1}+l_{2 i} h_{i+1}, \\
h_{i}-d_{3 i} & =-l_{3 i} g_{i+1}-l_{1 i} h_{i+1},
\end{aligned}
$$

localized at the maximal ideal generated by $(x, y, z)$. There is a natural homomorphism $\iota: B \rightarrow k[[x, y, z]]$. Indeed, we can use the relations (10.13) recursively in $i$ to construct homomorphisms $B \rightarrow \frac{k[x, y, z]}{(x, y, z)^{i}}$. By (10.13), $B$ is contained in a pure transcendental extension of $k(x, y, z)$ of degree 2 , generated by $f_{1}$ and $g_{1}$. Since $f_{1}$ and $g_{1}$ are mapped to elements of $k[[x, y, z]]$ algebraically independent over $k(x, y, z), \iota$ is injective.

Claim. The ring $B$ is noetherian. 
Proof of Claim. For $i \in \mathbb{N}$, let $B_{i}:=\frac{A\left[f_{i}, g_{i}, h_{i}\right]_{(x, y, z)}}{\left(l_{3 i}\left(f_{i}-d_{1 i}\right)+l_{1 i}\left(g_{i}-d_{2 i}\right)+l_{2 i}\left(h_{i}-d_{3 i}\right)\right)}$, so that $B=$ $\lim _{i \rightarrow \infty} B_{i}$ and $B_{i} \subset B$ for all $i \in \mathbb{N}$. By (10.13) all the $B_{i}$ are birational to each other (and hence also to $B$ ). Let $m:=(x, y, z) B$. By construction, for every $n \in \mathbb{N}$, $\frac{B}{m^{n}} \cong \frac{k[x, y, z]}{(x, y, z)^{n}}$, so that $k[[x, y, z]]$ is the $m$-adic completion of $B$. It is sufficient to prove that every prime ideal of $B$ is finitely generated. Let $I$ be a prime ideal of $B$ and put $I_{i}:=I \cap B_{i}$. Since $m$ is finitely generated, we may assume that $I \varsubsetneqq m$. Since $B_{i}$ is noetherian, $I_{i}$ is finitely generated. Hence it is sufficient to prove that for $i$ sufficiently large

$$
I_{i} B_{i+1}=I_{i+1}
$$

Let $\lambda_{i j}: B_{i} \rightarrow B_{j}$ denote the birational map between $B_{i}$ and $B_{j}$. There exist elements $v_{0 i j}, v_{1 i j}, v_{2 i j}, v_{3 i j} \in B_{i}$ such that $B_{j}=B_{i}\left[\frac{v_{1 i j}}{v_{0 i j}}, \frac{v_{2 i j}}{v_{0 i j}}, \frac{v_{3 i j}}{v_{0 i j}}\right]$. If $v_{0 i j} \notin I$ for all $j>i,(10.14)$ holds since in that case all the $\lambda_{i j}$ become isomorphisms after localization by the multiplicative system $S$ generated by the $v_{0 i j}, j>i$, so $I_{i} B_{i+1}=$ $I_{i}\left(B_{i+1}\right)_{S} \cap B_{i+1}=I_{i+1}\left(B_{i+1}\right)_{S} \cap B_{i+1}=I_{i+1}$. Now we compute $v_{0 i j}$ explicitly from (10.13) and show that we can choose $i$ sufficiently large so that $v_{0 i j} \notin I$. By $(10.10)$ and the identity $l_{3, i+1}\left(f_{i+1}-d_{1, i+1}\right)+l_{1, i+1}\left(g_{i+1}-d_{2, i+1}\right)+l_{2, i+1}\left(h_{i+1}-d_{3, i+1}\right)=0$, we have $v_{0 i, i+1}=l_{1 i} l_{1, i+1}-l_{2 i} l_{3, i+1}-l_{3 i} l_{2, i+1}$, which is a non-degenerate quadratic form in $x, y$ and $z$. Since $I \varsubsetneqq m$, there are at most two distinct values of $i$ for which $v_{0 i, i+1} \in I$. The Claim is proved.

Since $B$ is noetherian, it is a regular 3-dimensional local ring with residue field $k$; hence the inclusion homomorphism $A \rightarrow B$ is regular. By $(10.13), \Omega_{B / A}$ is exactly the module $M \otimes_{A} B$, where $M$ is the module of Example 10.5. If $B$ is a direct limit of smooth $A$-subalgebras $A_{\lambda}$ of finite type, these subalgebras would have transcendence degree two over $A$. Now assume, in addition, that char $k=0$. Then

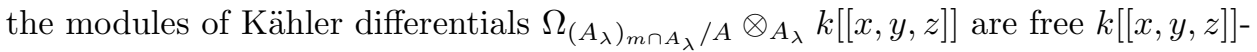
modules, injecting into $\Omega_{B / A} \otimes_{B} k[[x, y, z]]$. This is impossible by Example 10.5 (replace $k[x, y, z]_{(x, y, z)}$ in Example 10.5 by $\left.k[[x, y, z]]\right)$. Thus $B$ is not a direct limit of smooth $A$-subalgebras of finite type.

Similarly, one can use the example of Remark 10.8 to construct a regular homomorphism $\frac{k[x, y, z]_{(x, y, z)}}{\left(z^{2}-x y\right)} \rightarrow B$, where $B$ is a noetherian $A$-algebra of transcendence degree 1 over $A$ which is not a direct limit of smooth $A$-subalgebras of finite type.

\section{§11. Approximation theOREMS}

In this section, we use Theorem 1.1 to deduce the Artin approximation theorem for Henselian pairs, as well as the nested smoothing and nested Artin approximation theorem. First, we use Elkik's Lemma to strengthen Theorem 1.1 (under some additional hypotheses) by requiring that the smooth $A$-algebra $D$ of (1.2) be étale over a polynomial ring $A\left[v_{1}, \ldots, v_{k}\right]$.

Definition 11.1 (D. Popescu). Let $A$ be a ring and $D$ a smooth $A$-algebra of finite type over $A$. Let

$$
D=\frac{A[u]}{(f)},
$$

where $u=\left(u_{1}, \ldots, u_{n}\right)$ and $f=\left(f_{1}, \ldots, f_{r}\right)$, be a presentation. We say that (11.1) is standard if $D$ is a complete intersection (that is, ht $(f)=r$ ) and after 
renumbering the $f_{i}$ and the $u_{j}$, det $\left|\frac{\partial f_{i}}{\partial u_{j}}\right|_{1 \leq i, j \leq r}$ is invertible in $D$. If $D$ admits a standard presentation over $A$, we will say that $D$ is standard over $A$.

Note: if $D$ is standard, then it is étale over the polynomial ring $A\left[u_{r+1}, \ldots, u_{n}\right]$.

Proposition 11.2. Let $\sigma: A \rightarrow B$ be a ring homomorphism and $D$ a smooth finite type A-algebra together with an A-algebra homomorphism $\psi: D \rightarrow B$. Let $J$ be an ideal of $A$ such that

$$
\psi(D) \subset \sigma(A)+J B
$$

and every element of $(1+J B) \cap \psi(D)$ is a unit of $B$. Then there exists a finite rank projective $D$-module $M$, an element $x \in D^{\prime}:=S_{D} M$ and an A-algebra homomorphism $D_{x}^{\prime} \rightarrow B$, compatible with $\psi$, such that $D_{x}^{\prime}$ is standard over $A$. Given any presentation $D=\frac{A[u]}{I}, M$ can be chosen to be the direct sum of $\frac{I}{I^{2}}$ with a finite rank free D-module.

Proof. Let $D=\frac{A\left[u_{1}, \ldots, u_{n}\right]}{I}$ be a presentation of $D$. First, consider the $D$-algebra $S_{D} \frac{I}{I^{2}}$. By Lemma 5.2, $S_{D} \frac{I}{I^{2}}$ has a presentation in which the conormal bundle is globally trivial, that is, $S_{D} \frac{I}{I^{2}}$ is a complete intersection. Replacing $D$ by $S_{D} \frac{I}{I^{2}}$, we may assume that $D$ is a complete intersection, that is, in the new notation, $I=\left(f_{1}, \ldots, f_{r}\right)$, where $r=$ ht $I$. Under this assumption, we will show that $M$ can be chosen to be a free finite rank $D$-module.

Let $\frac{\partial f}{\partial u}$ stand for the $r \times n$ matrix $\left(\frac{\partial f_{i}}{\partial u_{j}}\right)$. Since $D$ is smooth over $A$, we have $\Delta_{f} D=D$. Then there exists an $n \times r$ matrix $G=\left(g_{i j}\right)_{\substack{1 \leq i \leq n \\ 1 \leq j \leq r}}$ with entries in $D$ such that

$$
\frac{\partial f}{\partial u} G=I d_{r}
$$

(where $I d_{r}$ stands for the $r \times r$ identity matrix). Let $t=\left(t_{1}, \ldots, t_{r}\right)$ be new variables and put $D^{\prime}=D[t]$. Extend $\psi$ to a map $\psi^{\prime}: D^{\prime} \rightarrow B$ in an arbitrary way, for example, by sending all the $t_{j}$ to 0 .

Now, for each $(i, j), 1 \leq i \leq n, 1 \leq j \leq r$, pick an element $a_{i j} \in A$ such that

$$
\sigma\left(a_{i j}\right) \equiv \psi\left(g_{i j}\right) \bmod J B
$$

(this is possible by (11.2)). Let $G_{0}$ denote the $n \times r$ matrix $\left(a_{i j}\right)_{1 \leq i \leq n}$. By (11.3) and (11.4), we have

$$
\psi\left(\frac{\partial f}{\partial u}\right) \sigma\left(G_{0}\right) \equiv I d_{r} \bmod J B
$$

so $\operatorname{det}\left(\psi\left(\frac{\partial f}{\partial u}\right) \sigma\left(G_{0}\right)\right) \equiv 1 \bmod J B$ and is therefore invertible. Let $x=\operatorname{det}\left(\frac{\partial f}{\partial u} G_{0}\right)$. Since $\psi(x)$ is invertible, $\psi^{\prime}$ extends to a map $D_{x}^{\prime} \rightarrow B$. It remains to show that $D_{x}^{\prime}$ is standard.

Consider the change of variables

$$
\tilde{u}_{i}=u_{i}-\sum_{j=1}^{r} a_{i j} t_{j}, \quad 1 \leq i \leq n .
$$


Let $\tilde{f}_{i}\left(\tilde{u}_{1}, \ldots, \tilde{u}_{n}\right):=f_{i}\left(u_{1}(\tilde{u}), \ldots, u_{n}(\tilde{u})\right), 1 \leq i \leq r$. By (11.6) and the chain rule,

$$
\operatorname{det}\left|\frac{\partial \tilde{f}_{i}}{\partial t_{j}}\right|_{l \leq i, j \leq r}=\operatorname{det}\left(\frac{\partial f}{\partial u} G_{0}\right)=x,
$$

which is invertible in $D_{x}^{\prime}$. Thus the Jacobian matrix of $\tilde{f}_{1}, \ldots, \tilde{f}_{r}$ has an $r \times r$ invertible minor, hence $D_{x}^{\prime}$ is standard over $A$. This completes the proof.

Now let $(A, I)$ be a Henselian pair and let $\hat{A}$ denote the $I$-adic completion of $A$. Assume that the natural homomorphism $\sigma: A \rightarrow \hat{A}$ is regular. This condition holds, for example, whenever $A$ is an excellent ring or, more generally, a G-ring. If $A$ is a local ring, then the requirement that $\sigma$ be regular is the definition of a G-ring. If $A$ is local and Henselian, $\sigma$ is regular if and only if $A$ is excellent. We obtain the following general form of the Artin approximation theorem.

Theorem 11.3. The approximation property holds for the pair $(A, I)$. That is, for a system of algebraic equations with coefficients in $A$, any solution in $\hat{A}$ can be approximated by a solution in A arbitrarily closely in the I-adic topology.

Proof. Consider a system of algebraic equations over $A$. This means that we are given unknowns $u_{1}, \ldots, u_{n}$ and a set of equations which we may regard as an ideal $J \subset A\left[u_{1}, \ldots, u_{n}\right]$. Consider the finitely generated $A$-algebra $C=\frac{A\left[u_{1}, \ldots, u_{n}\right]}{J}$. Consider a formal solution, that is, a homomorphism $\hat{\rho}: C \rightarrow \hat{A}$. We want to show that there exists a true solution, i.e. a homomorphism $\rho: C \rightarrow A$ which agrees with $\hat{\rho} \bmod I^{N}$, where $N$ is an arbitrarily fixed positive integer. By Theorem 1.1 and Proposition 11.2, we may assume that $C$ is an étale extension of the polynomial ring $A\left[u_{r+1}, \ldots, u_{n}\right]$ (where $r=$ ht $J$ ). We define the desired solution $\rho: C \rightarrow A$ as follows. For each $i, r<i \leq n$, pick $x_{i} \in A$ such that $\hat{\rho}\left(u_{i}\right)-x_{i} \in I^{N} \hat{A}$. Put $\rho\left(u_{i}\right)=x_{i}$. Now, $\frac{C}{\left(u_{r+1}-x_{r+1}, \ldots, u_{n}-x_{n}\right)}$ is an étale extension of $A$ having a section in $\frac{A}{I^{N}}$, hence it has a section in $A$ by the Henselian property of $(A, I)$.

A further generalization of Artin approximation is the nested approximation theorem. The nested version is useful for studying deformations of singularities, as, for example, in the work of Mostowski and Teissier on algebraization of singularities by deformation. We give two different proofs of the nested approximation. In one of them, we follow Bernard Teissier [19] in that we first prove Teissier's "nested smoothing" theorem and then deduce nested approximation as an easy corollary.

Theorem 11.4 (B. Teissier). Consider a commutative diagram

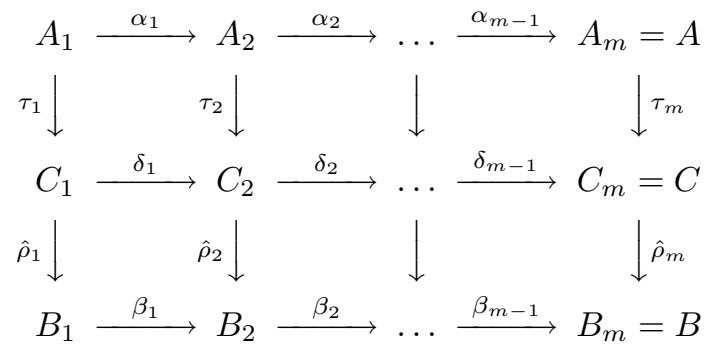

of homomorphisms between noetherian rings, where $C_{i}$ is of finite type over $A_{i}$, $1 \leq i \leq m$. Let $A_{i}^{\prime}:=A_{i} \otimes_{A_{i-1}} B_{i-1}\left(A_{1}^{\prime}=A_{1}\right.$ by convention $)$. Assume that $A_{i}^{\prime}$ 
is noetherian for all $i$. Assume that the induced homomorphism $\sigma_{i}: A_{i}^{\prime} \rightarrow B_{i}$ is regular for each $i$. Then (11.7) can be extended to a commutative diagram

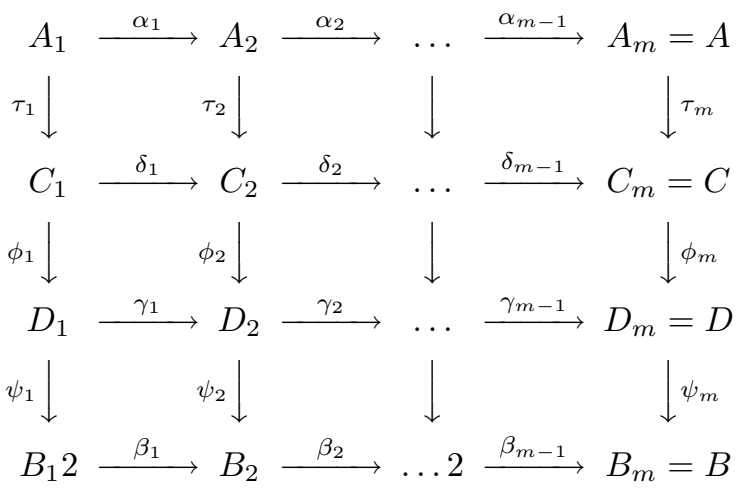

such that for each $i, 1 \leq i \leq m, D_{i}$ is smooth of finite type over $D_{i-1} \otimes_{A_{i-1}} A_{i}$ (for $i=1$, this condition should read "smooth over $A_{1}$ "). Let $I$ be an ideal of $A$ and $I_{i}:=I \cap A_{i}, 1 \leq i \leq m$. Assume that for each $i$ every element of $1+I_{i} B_{i}$ is a unit of $B_{i}$ and that $\frac{\bar{A}_{i}}{I_{i}} \cong \frac{B_{i}}{I_{i} B_{i}}$. Then $D_{i}$ can be chosen to be standard over $D_{i-1} \otimes_{A_{i-1}} A_{i}$.

Proof. We proceed by induction on $m$. For $m=1$ the result is nothing but Theorem 1.1 together with Proposition 11.2. Assume $m>1$. First, apply Theorem 1.1 and Proposition 11.2 to the maps $A_{m}^{\prime} \stackrel{\tau_{m}^{\prime}}{\longrightarrow} C \otimes_{A_{m-1}} B_{m-1} \stackrel{\hat{\rho}_{m}^{\prime}}{\longrightarrow} B$, where $\sigma_{m}^{\prime}=\hat{\rho}_{m}^{\prime} \circ \tau_{m}^{\prime}$ is regular by assumption and $C \otimes_{A_{m-1}} B_{m-1}$ is of finite type over $A_{m}^{\prime}$. We obtain a factorization

$$
A_{m}^{\prime} \rightarrow C \otimes_{A_{m-1}} B_{m-1} \rightarrow D^{\prime} \rightarrow B
$$

of $\hat{\rho}_{m}^{\prime}$, where $D^{\prime}$ is a smooth (resp. standard) $A_{m}^{\prime}$-algebra. Next, let $C_{m-1}^{\prime}$ be a finite type $\hat{\rho}_{m-1}\left(C_{m-1}\right)$-subalgebra of $B_{m-1}$ such that the first two homomorphisms in (11.9) are actually defined over $C_{m-1}^{\prime}$, so that the first two maps in (11.9) are obtained from a sequence

$$
A_{m} \otimes_{A_{m-1}} C_{m-1}^{\prime} \rightarrow C \otimes_{A_{m-1}} C_{m-1}^{\prime} \rightarrow \bar{D} \rightarrow B
$$

where $\bar{D}$ is a smooth (resp. standard) $\left(A \otimes_{A_{m-1}} C_{m-1}^{\prime}\right)$-algebra of finite type, by tensoring with $B_{m-1}$ over $C_{m-1}^{\prime}$. Consider the following commutative diagram:

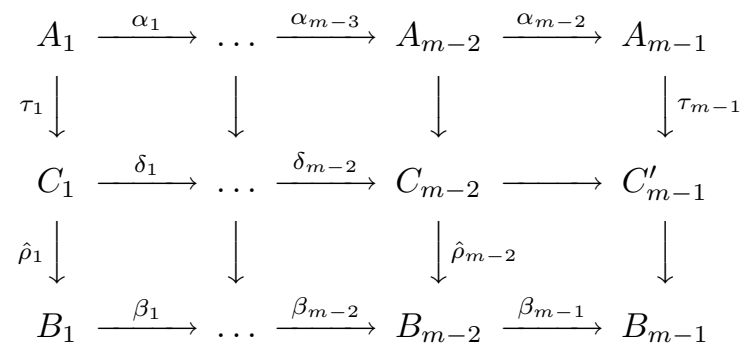


Apply the induction hypothesis to (11.11). We obtain a commutative diagram

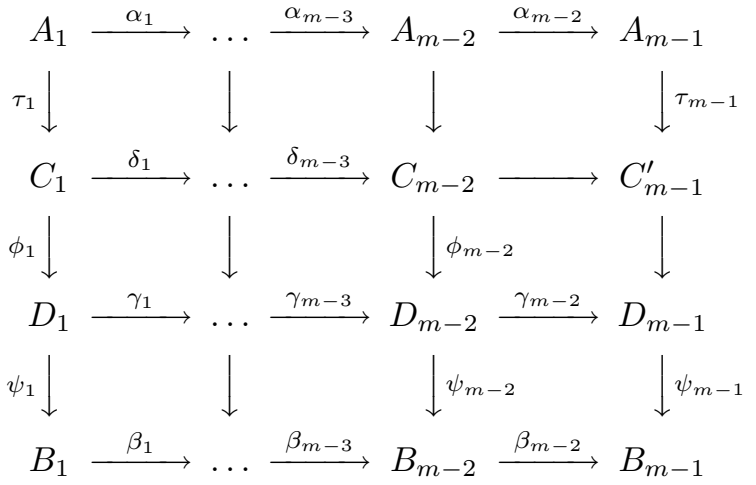

with $D_{i}$ is smooth of finite type (resp. standard) over $D_{i-1} \otimes_{A_{i-1}} A_{i}, i \leq m-1$. Put $D:=\bar{D} \otimes_{C_{m-1}^{\prime}} D_{m-1}$. Since $\bar{D}$ is smooth (resp. standard) over $A \otimes_{A_{m-1}} C_{m-1}^{\prime}, D$ is smooth (resp. standard) over $A \otimes_{A_{m-1}} D_{m-1}$. We have the map $D \rightarrow B$ coming from the tensor product; the map $C \rightarrow D$ is the composition $C \rightarrow C \otimes_{A_{m-1}} C_{m-1}^{\prime} \rightarrow$ $\bar{D} \rightarrow D=\bar{D} \otimes_{C_{m-1}^{\prime}} D_{m-1}$, where the first and last maps are given by $x \rightarrow x \otimes 1$. This completes the construction of (11.8).

Theorem 11.5. Consider a diagram (11.7). Let the assumptions be as in Theorem 11.4. Let $I$ be an ideal of $A$ and let $I_{i}:=I \cap A_{i}$. Let $\hat{A}_{i}$ denote the $I_{i}$-adic completion of $A_{i}, \hat{B}_{i}$ the $I_{i} B_{i}$-adic completion of $B_{i}$. Assume that

$$
\hat{A}_{i}=\hat{B}_{i} \quad \text { for all } i, 1 \leq i \leq m,
$$

and that $\left(A_{i}, I_{i}\right)$ is a Henselian pair for all $i$. Then there exists a homomorphism $\rho: C \rightarrow A$ such that for each $j, 1 \leq j \leq m-1, \rho\left(C_{j}\right) \subset A_{j}$. The homomorphism $\rho$ can be chosen to agree with $\hat{\rho}$ up to an arbitrarily specified power of $I$.

Remarks 11.6. (1) The algebras $C_{i}$ in the diagram (11.7) can be regarded as nested systems of algebraic equations over $A_{i}$ with solutions in $B_{i}$, which we want to approximate by solutions in $A_{i}$.

(2) Let \{\} denote convergent power series and \langle\rangle Henselization. The special cases of interest in Theorem 11.5 are $A_{j}=A_{1}\left\langle x_{1}, \ldots, x_{j}\right\rangle$ and $B_{j}=\hat{A}_{j}$ or $B_{j}=A_{1}\left\{x_{1}, \ldots, x_{j}\right\}$, where $A_{1}$ is equipped with a multiplicative norm in the sense of $[11, \S 45]$.

(3) If all the $A_{i}^{\prime}$ and $B_{i}$ are G-rings, as in (2) of this remark, the condition that the $\sigma_{i}$ be regular homomorphisms is a consequence of (11.13), so it does not need to be specified separately.

First proof of Theorem 11.5. By Theorem 11.4 we may, replacing the $C_{i}$ by the $D_{i}$, assume that each $C_{i}$ is standard over $A_{i} \otimes_{A_{i-1}} C_{i-1}$. Fix an integer $N \in \mathbb{N}$. We will denote by $\rho_{i}$ the restriction to $C_{i}$ of the hypothetical homomorphism $\rho$ which we want to construct. We will construct the maps $\rho_{i}: C_{i} \rightarrow A_{i}$ recursively in $i$. For $i=1, C_{1}$ is standard over $A_{1}$ and the map $\rho_{1}$ is constructed exactly as in Theorem 11.3. Suppose that $\rho_{i-1}$ has been constructed. Consider the decomposition

$$
\left(C_{i-1} \otimes_{A_{i-1}} A_{i}\right) \rightarrow\left(C_{i-1} \otimes_{A_{i-1}} A_{i}\right)\left[v_{1}, \ldots, v_{t}\right] \stackrel{\epsilon}{\rightarrow} C_{i},
$$

where the $v_{j}$ are algebraically independent over $C_{i-1} \otimes_{A_{i-1}} A_{i}$ and $\epsilon$ is étale. The map $\left(\alpha_{i-1} \circ \rho_{i}\right): C_{i-1} \rightarrow A_{i}$ induces a map $C_{i-1} \otimes_{A_{i-1}} A_{i} \rightarrow A_{i}$. We extend 
it to a map $\rho_{i}: C_{i} \rightarrow A_{i}$ in the usual way: first, let $\rho_{i}\left(v_{j}\right)$ be any element of $\left(\hat{\rho}_{i}\left(v_{j}\right)+I_{i}^{N} B_{i}\right) \cap A_{i}$, then extend $\rho_{i}$ to $C_{i}$ by definition of Henselian.

We give another proof of Theorem 11.5, under the additional assumption that the homomorphisms $\alpha_{i}$ are flat.

Second proof of the Nested Approximation Theorem, assuming that the $\alpha_{i}$ are flat. Again, we use induction on $m$. Again, consider the homomorphisms $A_{m}^{\prime} \rightarrow C \otimes_{A_{m-1}}$ $B_{m-1} \rightarrow B$. Since $(A, I)$ is a Henselian pair with $I$-adic completion $\hat{B}$, so is $\left(A_{m}^{\prime}, I A_{m}^{\prime}\right)$. Applying Theorem 11.3, we obtain a homomorphism $\rho^{\prime}: C \otimes_{A_{m-1}}$ $B_{m-1} \rightarrow A_{m}^{\prime}$ which agrees with $\hat{\rho} \bmod I^{N}$. We may regard $C$ as a $\left(C_{m-1} \otimes_{A_{m-1}} A\right)$ algebra; let $C=\frac{\left(C_{m-1} \otimes_{A_{m-1}} A\right)\left[v_{1}, \ldots, v_{l}\right]}{\left(g_{1}, \ldots, g_{s}\right)}$ be a presentation of $C$ over $C_{m-1} \otimes_{A_{m-1}} A$. For each $v_{i}$, write $\rho^{\prime}\left(v_{i}\right)=\sum_{j=1}^{k_{i}}\left(a_{i j} \otimes b_{i j}\right)$ with $a_{i j} \in A$ and $b_{i j} \in B_{m-1}$. Let $w=\left\{w_{i j} \mid 1 \leq i \leq l, 1 \leq j \leq k_{i}\right\}$ be new variables. Let $\sum_{j}\left(a_{j} \otimes b_{j}\right)$ stand for the collection $\left\{\sum_{j=1}^{k_{i}}\left(a_{i j} \otimes b_{i j}\right) \mid 1 \leq i \leq l\right\}$ and similarly for $\left\{\sum_{j}\left(a_{j} w_{j}\right)\right\} \subset A[w]$. For each $q, 1 \leq q \leq s$, let $h_{q}(w):=g_{q}\left(\sum_{j}\left(a_{j} w_{j}\right)\right) \in\left(C_{m-1} \otimes_{A_{m-1}} A\right)[w]$. Take a finitely generated $C_{m-1}$-submodule $M \subset C_{m-1} \otimes_{A_{m-1}} A$ which contains all the coefficients of all the polynomials $h_{q}$. Since $A$ is assumed flat over $A_{m-1}$, $C_{m-1} \otimes_{A_{m-1}} A$ is flat over $C_{m-1}$. Thus the inclusion $M \subset C_{m-1} \otimes_{A_{m-1}} A$ can be factored through a free $C_{m-1}$-module $F \cong C_{m-1}^{r}$. Let $\left\{e_{1}, \ldots, e_{r}\right\}$ be a $C_{m-1}$-basis of $F$. For $1 \leq q \leq s$, write $h_{q}(w)=\sum_{p=1}^{r} \bar{h}_{q p}(w) e_{p}$, where $\bar{h}_{q p}(w) \in C_{m-1}[w]$. Let $\bar{h}:=\left\{h_{q p} \mid 1 \leq q \leq s, 1 \leq p \leq r\right\}$. Put $\bar{C}_{m-1}:=\frac{C_{m-1}[w]}{(h)}$. Define a homomorphism $\bar{\rho}: \bar{C}_{m-1} \rightarrow B_{m-1}$ by setting $\bar{\rho}\left(w_{i j}\right):=b_{i j}$. We get a commutative diagram

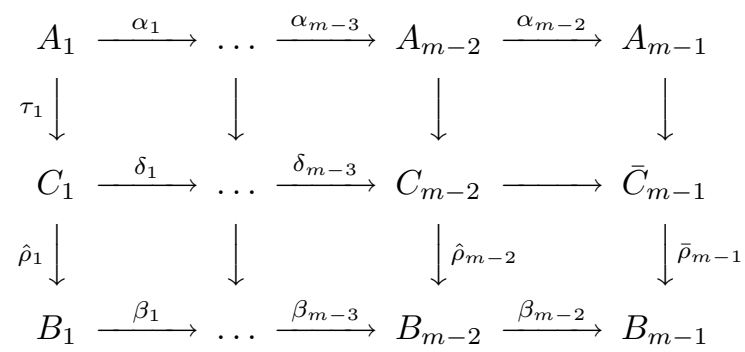

By the induction assumption, there exists a homomorphism $\rho_{m-1}: \bar{C}_{m-1} \rightarrow A_{m-1}$ which agrees with $\bar{\rho}_{m-1}$ modulo $I_{m-1}^{N}$ and such that $\rho_{m-1}\left(C_{i}\right) \subset A_{i}, 1 \leq i \leq m-1$. Now, letting $\rho\left(v_{i}\right):=\sum_{j=1}^{k_{i}} a_{i j} \rho_{m-1}\left(w_{i j}\right)$ defines a map $\rho: C \rightarrow A$ which agrees with $\hat{\rho}$ modulo $I^{N}$. This completes the proof.

\section{ApPendix. Regular homomorphisms Which are not injeCtive}

Let $\sigma: A \rightarrow B$ be a homomorphism of noetherian rings and let $I$ denote the kernel of $\sigma$. The purpose of this Appendix is to show that $\sigma$ is regular if and only if $A n n(I) B=B$ and the induced homomorphism $\frac{A}{I} \rightarrow B$ is regular.

Let $\bar{A}:=\frac{A}{I}$ and let $\pi: A \rightarrow \bar{A}$ be the natural map. 
Theorem A.1. Let $A$ be a noetherian ring and $I \subset A$ an ideal. Let $\bar{A}:=\frac{A}{I}$ and let $C$ be an $\bar{A}$-algebra. The following two conditions are equivalent:

(1) $C$ is regular over $A$.

(2) $C$ is regular over $\bar{A}$ and $A n n_{A}(I) C=C$.

Note that both (1) and (2) imply that $I$ is contained in a minimal prime of $A$.

Proof. There is a 1-1 correspondence between the primes of $\bar{A}$ and the primes of $A$ containing $I$. For any prime $P \subset \bar{A}$,

$$
\kappa\left(\frac{\bar{A}}{P}\right)=\kappa\left(\frac{A}{\pi^{-1}(P)}\right)
$$

so $\frac{B}{P B}$ is geometrically regular over one of these fields if and only if it is geometrically regular over the other. For a prime $P$ of $A$ not containing $I, \kappa\left(\frac{A}{P}\right) \otimes C$ is the zero ring, hence geometrically regular over $\kappa\left(\frac{A}{P}\right)$ by definition. Hence, to prove Theorem A.1, it is sufficient to prove

Lemma A.2. Let $A$ be $a$ ring and $I \subset A$ an ideal. Let $\bar{A}:=\frac{A}{I}$ and let $M$ be an $\bar{A}$-module. The following two conditions are equivalent:

(1) $M$ is A-flat.

(2) $M$ is $\bar{A}$-flat and $A n n_{A}(I) M=M$.

Note that if $A$ is noetherian, both (1) and (2) imply that $I$ is contained in a minimal prime of $A$.

Proof. (1) $\Longrightarrow(2)$. Since $M$ is $A$-flat, it is a direct limit of free $A$-modules. For each of the free $A$-modules $A^{n} \rightarrow M$ mapping to $M$, tensoring both sides with $\bar{A}$ over $A$, we get that $M$ is a direct limit of free $\bar{A}$-modules. Hence $M$ is $\bar{A}$-flat.

Since $M$ is an $\bar{A}$-module, every element in $M$ annihilates $I$. Thus to complete the proof of $(1) \Longrightarrow(2)$ it remains to prove

Sublemma A.3. Let $A$ be a ring, $I$ an ideal of $A$ and $M$ a flat $A$-module. Then the set of elements in $M$ annihilating I (which we denote by $A n n_{M}(I)$ ) is equal to $A n n_{A}(I) M$.

Proof. Let $a_{\lambda}, \lambda \in \Lambda$, be a set of generators of $I$. Consider the exact sequence

$$
0 \rightarrow A n n(I) \rightarrow A \rightarrow A^{\Lambda},
$$

where $A^{\Lambda}$ stands for the product of the (possibly infinite) set of copies of $A$ indexed by $\Lambda$ and the last map in (A.1) sends $x$ to $\left\{a_{\lambda} x\right\}_{\lambda \in \Lambda}$. Tensor (A.1) with $M$ and apply flatness. This completes the proof of $(1) \Longrightarrow(2)$.

$(2) \Longrightarrow(1)$. Now, assume that $M=A n n_{A}(I) M$ and that $M$ is $\bar{A}$-flat. Then $M$ can be written as a direct limit of free finitely generated $\bar{A}$-modules. We want to deduce that $M$ can also be written as a direct limit of free $A$-modules. For that it is sufficient to prove that any map $\bar{A} \rightarrow M$ can be factored through a free $A$-module:

$$
\bar{A} \rightarrow A^{n} \rightarrow M
$$

for some $n \in \mathbb{N}$. Giving a map $\rho: \bar{A} \rightarrow M$ is the same as giving an element $x \in M$ (where $x=\rho(1)$ ). Since $M=A n n_{A}(I) M$, there exist $y_{1}, \ldots, y_{n} \in A n n_{A}(I)$ and 
$x_{1}, \ldots, x_{n} \in M$ such that

$$
x=\sum_{i=1}^{n} y_{i} x_{i} .
$$

Then $\rho$ factors through $A^{n}$, where the map $\bar{A} \rightarrow A^{n}$ is given by sending 1 to $\sum_{i=1}^{n} y_{i} X_{i}$ (the $X_{i}$ being the generators of $A^{n}$ ) and $A^{n} \rightarrow M$ sends $X_{i}$ to $x_{i}$.

\section{REFERENCES}

1. M. André, Cinq exposés sur la désingularization, handwritten manuscript, École Polytechnique Fédérale de Lausanne, 1992.

2. M. André, Homologie des Algèbres Commutatives, Springer-Verlag, Berlin Heidelberg New York, 1974. MR 50:4707

3. M. Artin, Algebraic approximation of structures over complete local rings, Publ. Math. IHES 36 (1969), 23-58. MR 42:3087

4. M. Artin, Algebraic structure of power series rings, Contemp. Math. 13 (1982), 223-227. MR 84b: 13014

5. M. Artin and J. Denef, Smoothing a ring homomorphism along a section, Arithmetic and Geometry, Vol II, Birkhäuser, Boston, 1983, pp. 5-29. MR 84m:14007

6. M. Artin and C. Rotthaus, A structure theorem for power series rings, Algebraic Geometry and Commutative Algebra in Honor of Masayoshi Nagata (1987), 35-44. MR 90b:14006

7. R. Elkik, Solutions d'équations à coefficients dans un anneau hensélién, Ann. Sci. Éc. Norm. Super. $4^{e}$ sér. 6 (1973), 533-604. MR 49:10692

8. A. Grothendieck and J. Dieudonne, Élements de géometrie algébrique, Publ. IHES 20, 1964. MR 30:3885

9. S. Lang, Algebra, Addison-Wesley, Reading, Massachusetts, 1967. MR 33:5416

10. H. Matsumura, Commutative Algebra, Benjamin, New York, 1970. MR 42:1813

11. M. Nagata, Local Rings, Krieger Publishing Co., Huntington, N.Y., 1975. MR 57:301

12. V. Nica and D. Popescu, A structure theorem on formally smooth morphisms in positive characteristic, J. of Algebra 100 (1986), 436-455. MR 87j:14007

13. T. Ogoma, General Néron desingularization based on the idea of Popescu, J. of Algebra 167 (1994), 57-84. MR 96a:13008

14. D. Popescu, General Néron desingularization, Nagoya Math. J. 100 (1985), 97-126. MR 87f: 13019

15. D. Popescu, General Néron desingularization and approximation, Nagoya Math. J. 104 (1986), 85-115. MR 91e:14003

16. D. Popescu, General Néron desingularization and approximation, Nagoya Math. J. 104 (1986), 85-115. MR 88a:14007

17. C. Rotthaus, On the approximation property of excellent rings, Invent. Math. 88 (1987), 39-63. MR 88c:14005

18. R. Swan, Néron-Popescu desingularization, to appear, Proceedings of the International Conference on Algebra and Geometry, Taipei, Taiwan (1995).

19. B. Teissier, Résultats récents sur l'approximation des morphismes en algèbre commutative, [d'après Artin, Popescu, André et Spivakovsky], Séminaire Bourbaki 784 (1994), 1-15. MR 96c: 13023

Department of Mathematics, University of Toronto, Erindale College, 3359 MissisSauga Road, Mississauga, Ontario, Canada L5L 1C6

E-mail address: spiva@math.toronto.edu 